\title{
Optimizing fMRI analysis in Schizophrenia research: methodology improvements
}

\author{
Juan José Lull Noguera
}

PhD Supervisors:

Montserrat Robles Viejo

Luis Martí-Bonmatí

José Vicente Manjón Herrera

PhD thesis submitted to the Departamento de Física Aplicada, Universidad Politécnica de Valencia, to obtain the Doctoral Degree.

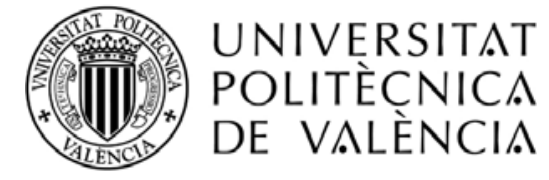

Valencia, 2008 


\section{Index}

i Agradecimientos ,................................................................

ii Abstract ........................................................................ vii

iii Resumen ...................................................................... viii

iv Resum ............................................................................ ix

1. Introduction ............................................................................... 1

a. Joint multidisciplinary research preamble

b. fMRI technique for non-invasive brain function monitoring

c. Schizophrenia illness research

2. Thesis motivation and objectives
a. Assumptions
b. Hypothesis
c. Thesis contributions
d. Thesis outline

3. Emotional auditory paradigm in neuroimaging: a base for the study of psychosis

a. Quality assessment of the acquired data

b. Data analysis

c. General Linear Model

d. Multiple comparison testing

e. Implementation of the data analysis

f. SPM software

g. PhD candidate contributions

h. Addendum

4. Emotional Words Induce Enhanced Brain Activity in Schizophrenic Patients with Auditory Hallucinations

a. Auditory paradigm 

b. Emotion and schizophrenia
c. Emotional words. An emotional semantic auditory paradigm
d. Data analysis
e. Process automation
f. PhD candidate contributions
g. Addendum
h. Supplementary results

5. MR Analysis of the Coincidence between Functional and Morphological Abnormalities in Schizophrenic Patients with Chronic Auditory Hallucinations 69
a. Multimodal image analysis
b. Other multimodality analysis experiments
c. PhD candidate contributions
d. Comment to the manuscript and addendum

6. $\mathrm{fMRI}$ activation localization enhanced by Non Local Means filtering 85

a. PhD candidate contributions

b. Addendum

c. Supplementary results

7. Concluding remarks and future research direction
a. Compilation of results
b. Conclusion
c. Future research direction

Annex i. Abreviations ............................................................. 133

Annex ii. Publications ............................................................... 135

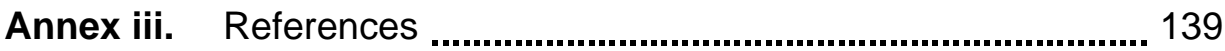




\section{Agradecimientos}

"La educación es la mejor provisión para la vejez" -Aristóteles (384-322 a.d.C.)

Una tesis es siempre un trabajo arduo. Conlleva mucha labor, quebraderos de cabeza y un camino de aprendizaje. Por el último motivo mencionado, en primer lugar me gustaría agradecer a mis directores de tesis, Montse, Luis y Josevi, el tiempo que me han dedicado. En buena medida ese tiempo ha sido de formación desinteresada, con un espíritu de difusión del conocimiento y del saber hacer que me han llenado de satisfacción. A ellos les doy las gracias por introducirme en el mundo de la investigación.

La tesis conlleva asperezas que se llevan con mucho menos peso cuando se puede compartir un café con los colegas. Agradezco ahora su tiempo, en numerosas ocasiones de ayuda, y también de café, a Josete, Mariam, Gracián, Alejandro, María José y Antonis, del Área de Imagen. Y también a todos los de Patrones y Sistemas de Información, a quienes empecé conociendo mediante los partidos de squash...

Cinco años de trabajo es un período en el que se conoce a mucha gente. Agradezco ahora a los que han facilitado que esta tesis haya sido un trabajo multidisciplinar.

Finalmente... iqué importante es la familia! Siempre te están ayudando, aunque uno ya se haya alejado de los 18, aunque ya no sea un niño. Agradezco el apoyo sincero de mis padres, hermanas, cuñados... y también a Churro, a los "Javis", Damián, Pep, Santiago... A todos, su cariño que siempre ha estado muy por encima de lo que yo merecía. 


\section{Abstract}

Functional Magnetic Resonance Imaging (fMRI) is a modern technique for neuroimaging that has the ability of localizing neural activity with a high spatial resolution. The fMRI technique utilizes local blood oxygenation changes, which are reflected as small intensity changes in a special type of Magnetic Resonance images. Its ability to detect changes in function in the healthy and unhealthy brain and localization of abnormal function makes it an ideal technique in the treatment follow-up of many neural illnesses and lesions. It has already been applied clinically for the localization of functional areas affected by tumours, pre- and postoperatively.

Schizophrenia, a major illness, present in more than the one percent of the whole population, is an illness that has been recently studied with functional neuroimaging, with more than 300 peer-reviewed journal papers about fMRI and schizophrenia. Understanding the neural substrates of schizophrenia requires a precise determination of the extent and distribution of abnormalities in brain anatomy and function. Due to the widespread distribution of symptoms, a defined phenomenological approach to this disease should be used in order to precisely relate abnormalities, symptoms and prognosis. Patients with dominant positive symptoms, such as auditory hallucinations and delusions, may have different brain abnormalities than those with marked negative symptoms. Thus, presence of auditory hallucinations in schizophrenic patients is taken in this study as the criterion for the selection of a homogeneous group of auditory hallucinatory schizophrenic patients.

This thesis presents the application of fMRI to study the schizophrenia illness. Finally, a new method for filtering fMRI data, NL-means, has been proposed, and it is suggested to be used as part of the pre-processing in fMRI studies. 


\section{Resumen}

La Resonancia Magnética funcional (RMf) es una técnica moderna de neuroimagen que permite la localización de actividad neuronal con una alta resolución espacial. La técnica de RMf emplea los cambios locales de oxigenación en la sangre, reflejados como pequeños cambios en la intensidad en un tipo concreto de imagen de Resonancia Magnética. La habilidad de esta técnica en la detección de cambios en la función en el cerebro sano y enfermo, y la localización de función anormal convierte a la RMf en una técnica ideal para el tratamiento de numerosas enfermedades y lesiones neuronales. Ya se ha aplicado clínicamente en la localización de áreas funcionales afectadas por tumores, pre- y postoperativamente.

La esquizofrenia, una vasta enfermedad que se encuentra presente en el uno por cien de la población global, es una dolencia que se ha estudiado recientemente mediante técnicas de neuroimagen funcional, con más de 300 estudios publicados en revistas sobre esquizofrenia y RMf. La comprensión de los sustratos neuronales de la esquizofrenia requiere una determinación precisa de la extensión y la distribución de anormalidades en la función y anatomía cerebrales. Ya que los síntomas tienen una distribución dispersa, se debería emplear una aproximación fenomenológica a esta enfermedad para relacionar anormalidades, síntomas y prognosis con precisión. Los pacientes que tienen principalmente síntomas positivos, tales como alucinaciones auditivas y delirios, pueden tener anomalías cerebrales diferentes a aquellos que tienen síntomas negativos pronunciados. Por tanto, en el presente estudio se ha seleccionado un síntoma positivo, la presencia de alucinaciones auditivas en pacientes esquizofrénicos, como el criterio de selección de un grupo homogéneo de pacientes esquizofrénicos auditivos.

Esta tesis presenta la aplicación de la RMf al estudio de la enfermedad de la esquizofrenia. Finalmente, un nuevo método de filtrado de datos de RMf, el NLmeans, se ha propuesto y se sugiere su uso como parte del pre-proceso en estudios de RMf. 


\section{Resum}

La Ressonància Magnètica funcional (RMf) és una tècnica moderna de neuroimatge que permet la localització d'activitat neural amb una alta resolució espacial. La tècnica de RMf servix els canvis locals d'oxigenació en la sang, reflectits com a menuts canvis en la intensitat en un tipus concret d'imatge de Ressonància Magnètica. L'habilitat d'esta tècnica en la detecció de canvis en la funció en el cervell sa i malalt, i la localització de funció anormal convertix la RMf en una tècnica ideal per al tractament de nombroses malalties i lesions neurals. Ja s'ha aplicat clínicament en la localització d'àrees funcionals afectades per tumors, pre- i post-operativament.

L'esquizofrènia, una vasta malaltia que es troba present en l'u per cent de la població global, és una malaltia que s'ha estudiat recentment per mitjà de tècniques de neuroimatge funcional, amb més de 300 estudis publicats en revistes sobre esquizofrènia i RMf. La comprensió dels substrats neurals de l'esquizofrènia requerix una determinació precisa de l'extensió i la distribució d'anormalitats en la funció i anatomia cerebrals. Ja que els símptomes tenen una distribució dispersa, s'hauria d'emprar una aproximació fenomenològica a esta malaltia per a relacionar anormalitats, símptomes i prognosi amb precisió. Els pacients que tenen principalment símptomes positius, com ara al-lucinacions auditives i deliris, poden tindre anomalies cerebrals diferents d'aquells que tenen símptomes negatius pronunciats. Per tant, en el present estudi s'ha seleccionat un símptoma positiu, la presència d'al-lucinacions auditives en pacients esquizofrènics, com el criteri de selecció d'un grup homogeni de pacients esquizofrènics auditius.

Esta tesi presenta l'aplicació de la RMf a l'estudi de la malaltia de l'esquizofrènia. Finalment, un nou mètode de filtrat de dades de RMf, el NL-means, s'ha proposat i se suggerix el seu ús com a part del preprocés en estudis de RMf. 


\section{Introduction}

"There is no scientific study more vital to man than the study of the human brain. Our entire view of the universe depends on it." --Frances H.C. Crick, 1979

\section{1.a - Joint multidisciplinary research preamble}

This dissertation involves the application of functional magnetic resonance (MR) imaging (fMRI) to schizophrenia research.

fMRI is a technique that consists in the use of MRI to measure the haemodynamic response related to neural activity in the central nervous system, in humans or animals. It is one of the most recently developed forms of neuroimaging. $\mathrm{fMRI}$ is a non-invasive technique that measures brain activation, with high spatial and temporal resolution.

On the other hand, schizophrenia is a major psychiatric illness, which affects around $1 \%$ of the world population. Language, thought and social disorders are symptoms present in schizophrenia. fMRI is currently being used to investigate how the neural networks in the unhealthy brain activate when the subject is performing a task or being stimulated. fMRI has been successfully used to study the way that different stimuli engage brain activation, such as visual, auditory, language-processing, language-recognition, olfactory, working memory, emotional, and many other different kinds of stimuli.

How the brain works is still mostly unknown nowadays. Advanced knowledge of the technique is needed, so that brain activation, haemodynamics and schizophrenia illness are jointly evaluated. A proper paradigm should be created that generated the expected haemodynamic response related to neural activity; many other experimental variables should be taken into account, like stimuli delivery rate (since subjects will get used to the presentation of stimuli), task performance or movement related to task. Interpretation of the fMRI results is difficult too. Care should be taken when making inferences from fMRI results (i.e. fMRI activation maps). 
A project that studies schizophrenia by means of $\mathrm{fMRI}$ involves researchers from different areas. Engineers have to supply their skills for the highly complex acquisition and analysis processes involved. Psychiatrists look at the symptoms in schizophrenia. MR images are to be obtained and therefore radiologists are also involved. A psychophysiology background is also needed. Hence a clear hypothesis can be proposed and tested. This hypothesis will be about the areas that are activated when the subject is stimulated and what differences should be expected in the activation between schizophrenic patients and healthy subjects. All these fields generate, and work with, very different and multispectral information. Furthermore, a specific terminology is employed differently by radiologists, psychologists, psychiatrists, engineers, etc. so the combination of the different work fields must be active. The synergy of work should be done by computer science engineers that could relate all information sources.

The PhD candidate has been involved in a schizophrenia research project, that started in 2003. The project consists of the study of schizophrenia by clinical assessment, by neuroimaging (structural MR imaging, fMRI, Magnetic Transfer imaging, Diffusion Weighted imaging and spectroscopy) and genetic characterization of a) a group of schizophrenic patients with auditory hallucinations; b) a group of schizophrenic patients without hallucinations; and c) a group of healthy subjects. The project has been funded since 2005 until 2008 by the Carlos III Institute of the Spanish Health Ministry, Ministerio de Sanidad y Consumo. Neuroimaging, genetic and clinical data have been acquired from more than ninety subjects since 2003 .

As a concluding remark, it is worth noting that fMRI is a very promising non-invasive technique that can shed light to our knowledge regarding schizophrenia. However there are some complex topics that affect fMRI studies. A suitable paradigm should be designed with a correct acquisition sequence; the haemodynamic-related-to-neural activation signal should not be lost but it should not be saturated either; the paradigm should maximize neural activity detection. Acquisition is also potentially problematic. Movement due to the task, e.g. in an overt-speaking paradigm, would spoil all the acquired data. Analysis of the acquired fMRI data comprises many different steps; as an example, movement should be corrected even in the case that it was lower than $0.5 \mathrm{~mm}$ during the fMRI acquisition. Finally, interpretation of the results should be carefully assessed. 
A schizophrenia brain activation estimation project has been carried out with all the aforementioned variables taken into account and its results are presented in this dissertation, along with different processes that are proposed to enhance the fMRI studies.

\section{1.b - fMRI technique for non-invasive brain function monitoring}

fMRI is a non-invasive technique. The main goal in $\mathrm{AMRI}$ is to locate brain activity. fMRI uses an MRI specific sequence, typically an Echo Planar Imaging (EPI) sequence (conceived by Mansfield, 1977). The first fMRI reports were given by Ogawa et al. (1990, 1993), first in rodents and then in human subjects. After those initial results, an ever growing interest in this technique has been shown.

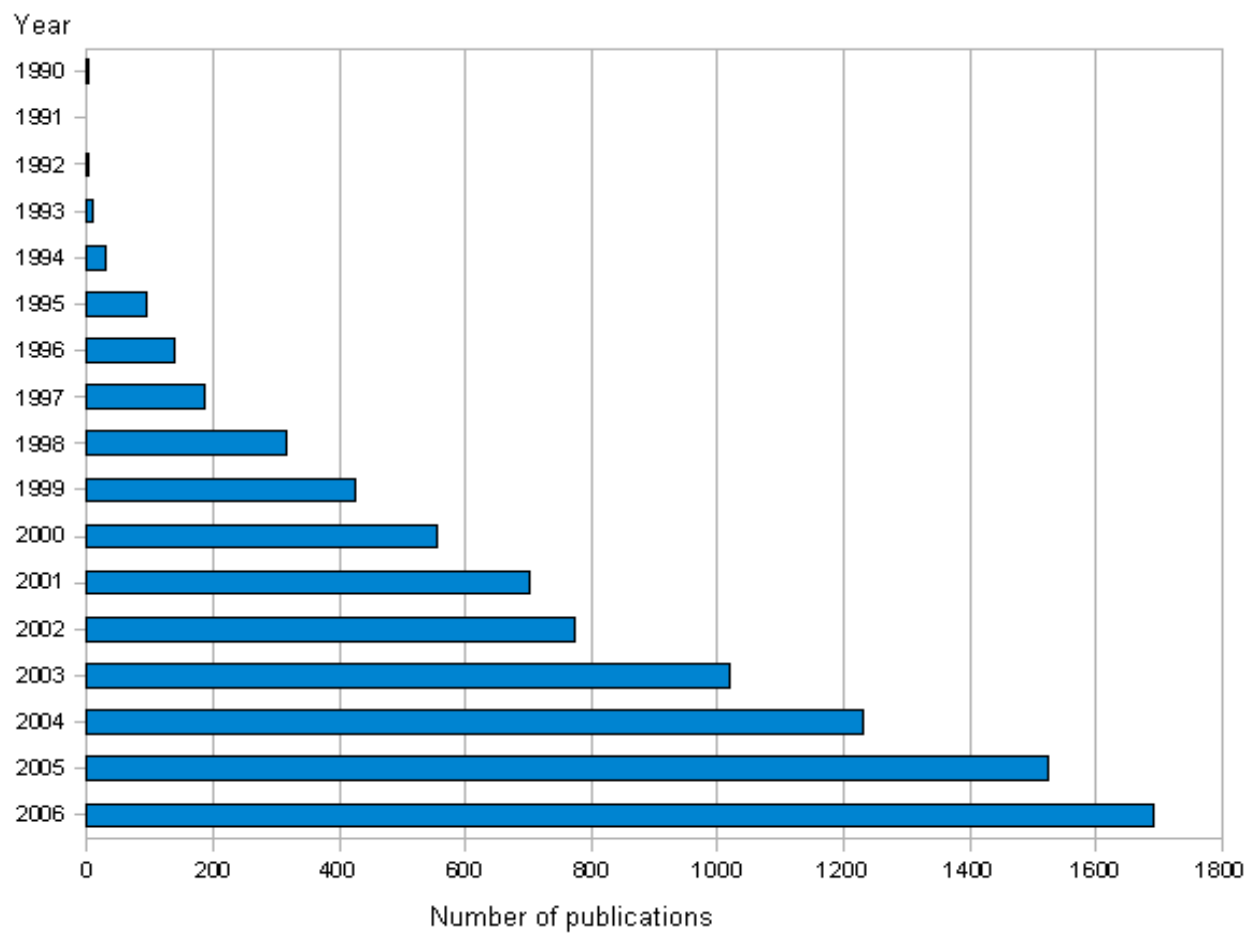

Figure 1. fMRI papers present in the PubMed database. Search performed with the sentence "fMRI" or "functional MRI".

Figure 1 illustrates the growth in number of scientific papers, which has increased exponentially since 1990 (for a more detailed review of the growth in the field, see Bandettini, 2007). The reasons for this outburst in fMRI research are many, but two of them are particularly important. First, fMRI is a non-invasive 
harmless technique. Second, spatial resolution is high in fMRI compared to other techniques such as Electroencephalography (EEG) and Positron Emission Tomography (PET). Furthermore, fMRI examinations can be carried out using standard clinical MR scanners which are available at nearly all modern hospitals and the procedure is completely painless.

With the establishment of fMRI as a brain activity mapping tool, the neuroscience field has also experienced a boost due to the possibility of studying human brain function in a harmless way. The relationship between brain activity and low level functions, such as basic sensory, visual and motor brain functions as well as higher cognitive functions such as language and memory have been tackled in many experiments. Another important application has been pre-surgical examinations, e.g. prior to the removal of a tumor, where the brain functions located in the regions surrounding the tumor can be mapped and the surgical approach can be optimized based on this information. The use of fMRI in presurgical applications is near its clinical validation (Sunaert, 2006).

The fMRI technique relies on blood flow and blood oxygen concentration (jointly known as haemodynamics) as indicators of brain activity. Local blood flow changes in active brain areas were predicted already by the end of the 19th century (Roy and Sherrington, 1890). The physiological basis of fMRI and how brain activity can be detected in MR images are further described below.

\section{The BOLD signal}

Neurons in the brain consume oxygen that is attached to haemoglobin molecules in the blood. The flow of blood continuously provides new oxygen to the neurons.

When neuronal activity increases, the demand of oxygen also rises. To meet this demand, an increased flow of blood is regionally supplied to the population of active neurons. Even though one would expect that the oxygenated blood rate would decrease when localized brain function appears, the situation is the opposite.

Oxygen is transported from the interior of haemoglobin cells to the plasma, then to extra-vascular space, to the intra-cellular space, and finally reaches the 
interior of the mitochondria via a pressure gradient (Buxton et al, 1998). To increase this pressure gradient it is necessary to increase the local concentration of oxygenated haemoglobin in the blood. Consequently, although there is an increase in oxygen consumption, there is a bigger increase in oxygen supply, creating a higher oxyhaemoglobin / deoxyhaemoglobin concentration than in rest state. This leads to an increase in the signal in BOLD images.

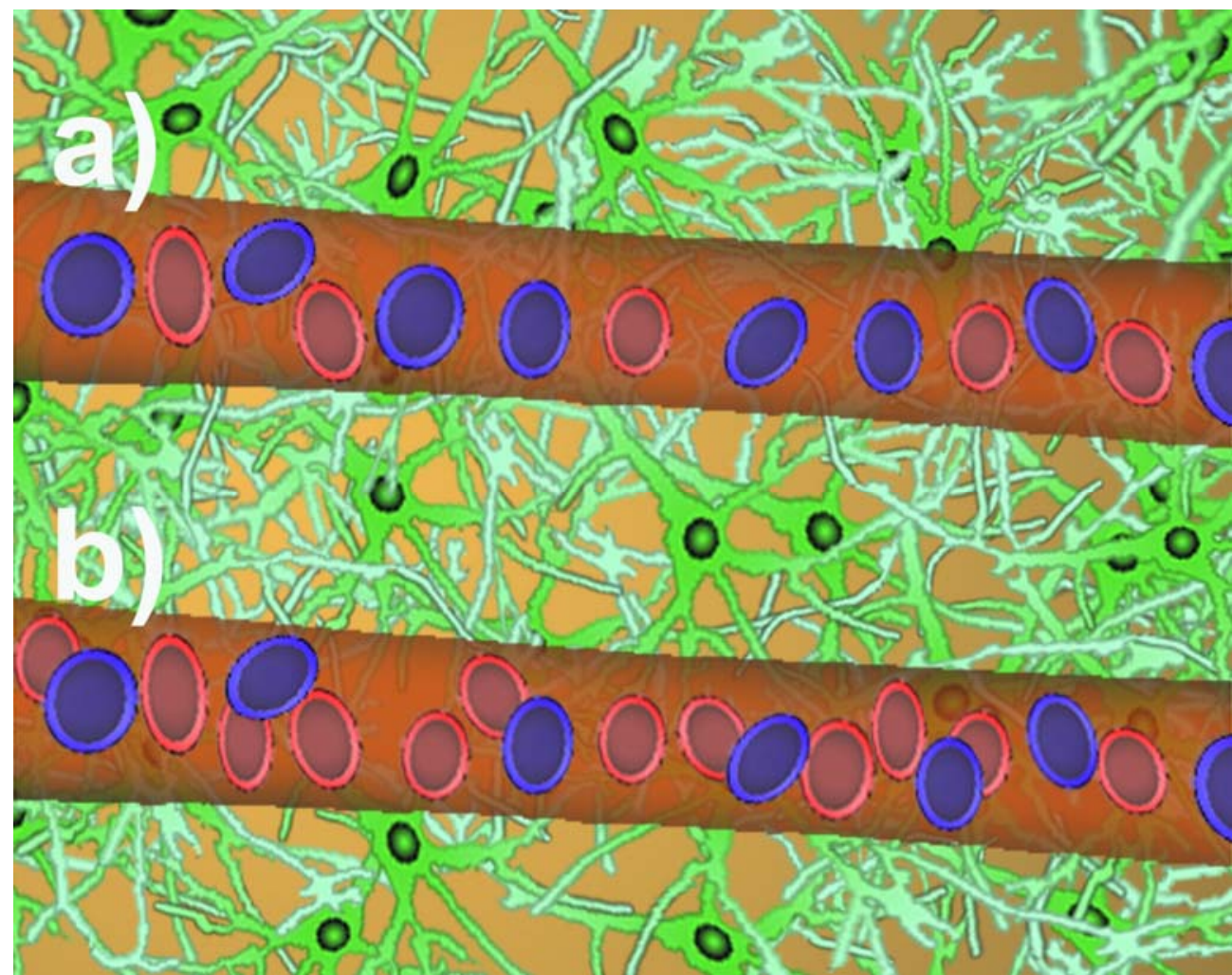

Figure 2. The BOLD signal reflects the proportion of oxygenated haemoglobin (red circles) and deoxygenated haemoglobin (blue circles) that are present in the capillaries. When in an active state (b), neurons consume oxygen and more oxygen than needed is supplied. This results in an increased inflow of oxygenated blood (b) compared to baseline state (a). Red-coloured cells represent oxyhaemoglobin while blue cells represent deoxy-haemoglobin.

The mechanisms underlying this very local regulation of blood flow are not yet fully understood. An excess of oxygen is always supplied to the active neurons, leading to an increased concentration of oxygenated blood in the capillaries surrounding the active brain area. This process is illustrated in Figure 2. It is this difference in oxygenation concentration between a baseline state and an active 
state that can be measured with an MR scanner due to the different magnetic properties of oxygenated blood and deoxygenated blood. When oxygen is attached to the haemoglobin molecule an iron atom is shielded. In this state, the haemoglobin molecule is slightly diamagnetic and therefore almost magnetically inactive. Without oxygen attached, the haemoglobin's iron is exposed and the molecule becomes paramagnetic, which means that it interacts with, and distorts, an applied magnetic field. The oxygen concentration in the blood therefore affects the magnetic environment of the hydrogen nuclei that exist in the water molecules.

At low oxygen concentrations there are many paramagnetic haemoglobin molecules that locally modulate the main magnetic field $B_{0}$ and as a consequence make the hydrogen nuclei excited by an RF-pulse dephase faster. Hence, the T2* relaxation time becomes shorter in areas with low oxygen concentration while it becomes longer in areas with high oxygen concentration.

MR images reflecting the T2* effect are therefore slightly brighter (longer T2*) when a brain area is in an active state compared to the baseline state. This effect is referred to as the Blood Oxygen Level Dependent (BOLD) signal. The effect is however very small: An intensity change of around two to five percent is expected, so changes due to the BOLD signal are not detectable visually.

The BOLD signal is an indirect indicator of brain activity and an important question is how well it corresponds to the electrical neuronal activity, generally taken as the definition of brain activity. In a seminal article, Logothetis and colleagues (Logothetis et al, 2001) showed by simultaneous measurements of electrical activity and blood oxygenation that these are intimately coupled. However, it is not possible to measure the activity of single neurons by means of fMRI due to: Firstly, a large population of neurons is required to evoke a measurable BOLD response. And secondly, the BOLD signal-to-noise ratio is higher the bigger the voxel, so signal change is measurable if voxels are at least approximately $1 \mathrm{~mm}^{3}$ (Menon and Goodyear, 1999). Typical voxel size in fMRI studies is about $10-30 \mathrm{~mm}^{3}$, though (in the studies presented in this PhD, voxel size is $14,8 \mathrm{~mm}^{3}$ ). 


\section{Detecting brain activity}

To perform an fMRI experiment, an MR scanner with the ability to acquire EPI images is required. A subject, either patient or volunteer, is placed in the scanner and functional images covering a volume of the brain are then continuously acquired during a period of 5 to 10 minutes, usually (along the text of this thesis, scanning time is set to 5 minutes and 20 seconds).

The in-plane size of the EPI images may be $64 \times 64$ or $128 \times 128$ voxels and a stack of $10-40$ slices (128x128 matrix; 24 slices in the presented studies) are generally acquired. Around 100-200 (120 in this case) such image volumes are then repeatedly collected during the examination with a sampling period commonly between 1 and 5 seconds (repetition time is 2 seconds in the currently presented studies).

While the images are acquired, the subject is either instructed to perform a task or presented with some form of stimuli. For example, a visual stimulus can be presented on a screen during a period of 20 seconds (an experimental block) and then a blank screen will be shown for 20 seconds (another block). Then the sequence of these two blocks is repeated throughout the whole imaging session. Due to the BOLD signal effect, there will be a BOLD response in brain areas that are activated by the presented visual stimulus. Such areas will presumably be located in the visual cortex. Hence, in an EPI image collected while a visual stimulus is being presented to the subject, higher image intensity values should be present in voxels covering active brain areas compared to the intensities in the same voxels in an image collected during the resting period. As the same image slice has been acquired repeatedly during the experiment, there is a time series of intensity values for each voxel, as shown in Figure $\mathbf{3}$. In a voxel covering a brain area participating in the processing of the presented visual stimulus, we expect a BOLD response, i.e. a variation in the time series following the pattern of the stimulus presentation. To locate and create a map of the active brain areas, voxels whose time series contain a BOLD response component must be identified. 


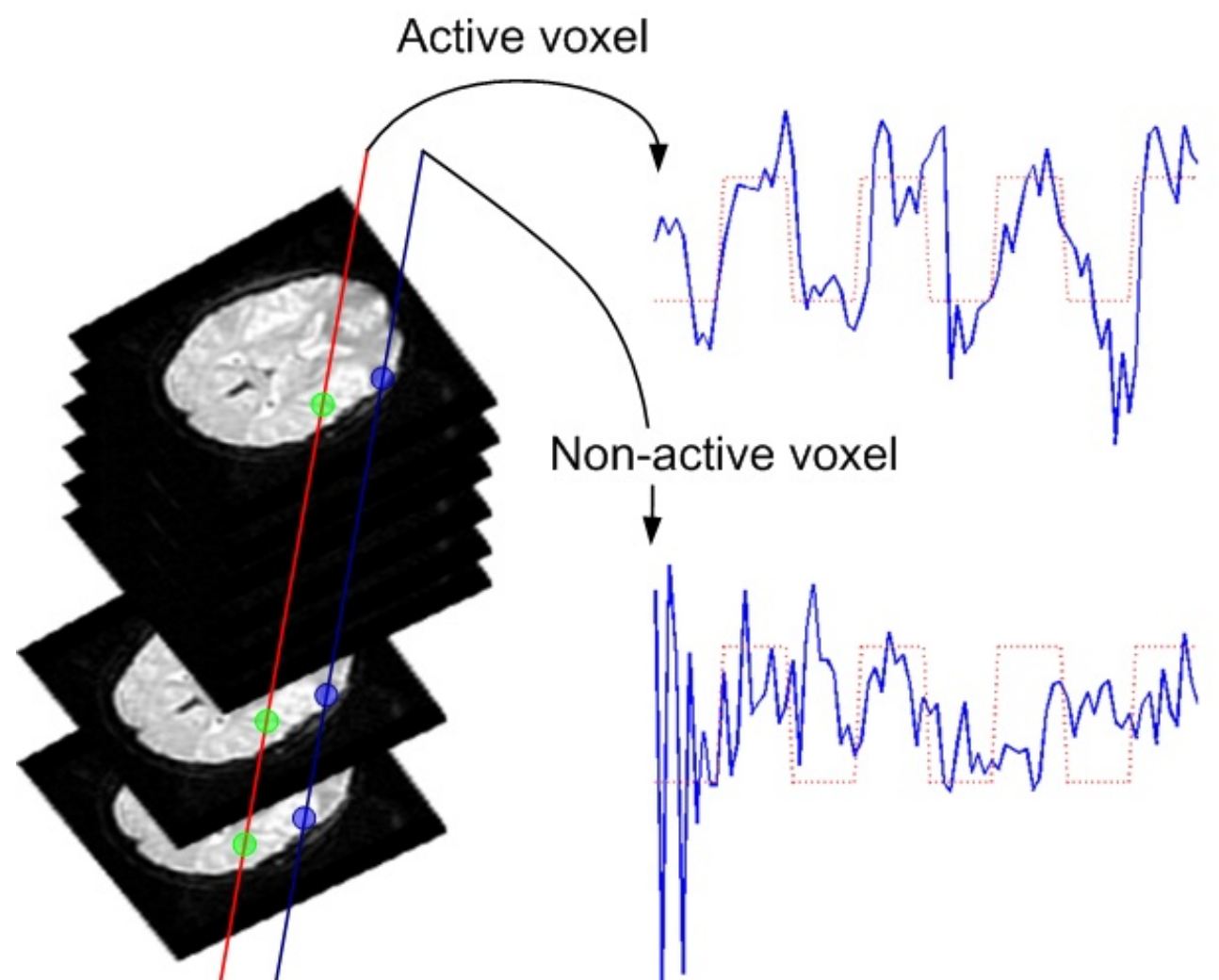

Figure 3. The same image slice is repeatedly collected during a period of 5 to 10 minutes. A time series of intensity values is therefore obtained in each voxel. Time series in active brain voxels contain a BOLD response while time series in nonactive voxels contain only noise (blue lines; time-courses correspond to voxels from acquired fMRI data). The rest and activity periods are indicated by the dotted lines.

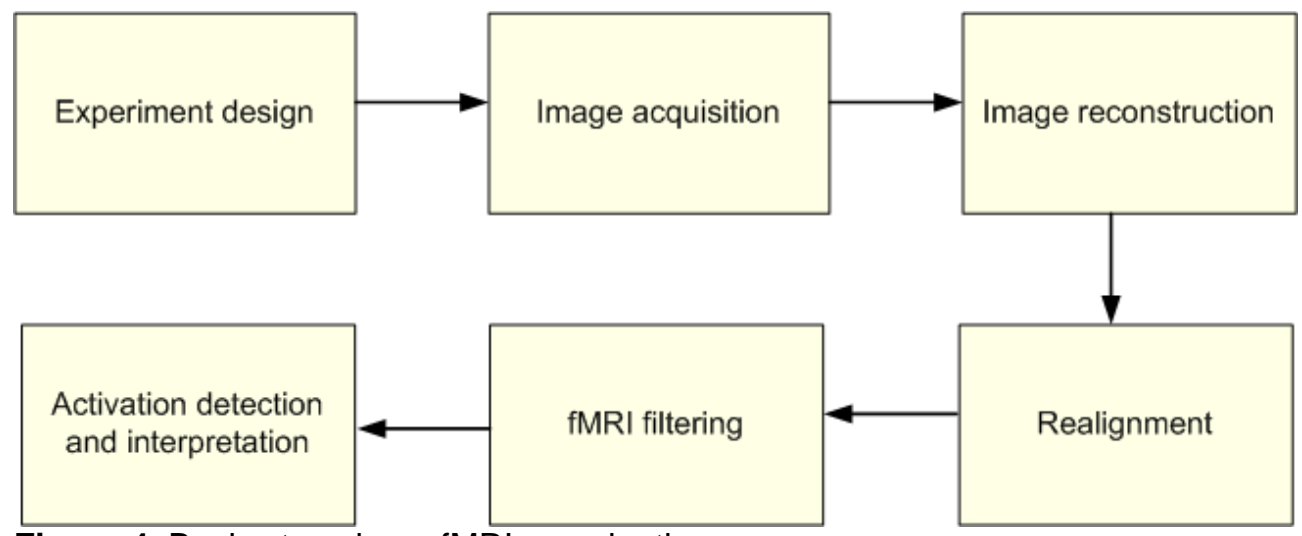

Figure 4. Basic steps in an fMRI examination. 
The process in an fMRI examination (Figure 4) starts, in general, with the experiment design. The experimental paradigm can either be a blocked design or an event-related design. In a blocked design, sustained brain activity is evoked by presenting a stimulus for a longer period, and then a control or resting block is used to contrast the activity block. The visual stimulus example above is of a blocked form. The BOLD response to a blocked experiment has a form similar to a square-wave as shown in Figure 5. If the stimulation is applied in a square wavefashion (red dotted line), then we would expect to find the square wave convolved by an ideal canonical haemodynamic function (blue line). In an event-related design stimuli are briefly shown. For example, familiar and unfamiliar faces can be briefly shown with irregular time spacings. The event-related design offers more flexibility but in return the BOLD response is smaller and more complicated to analyze (Friston et al, 1999).

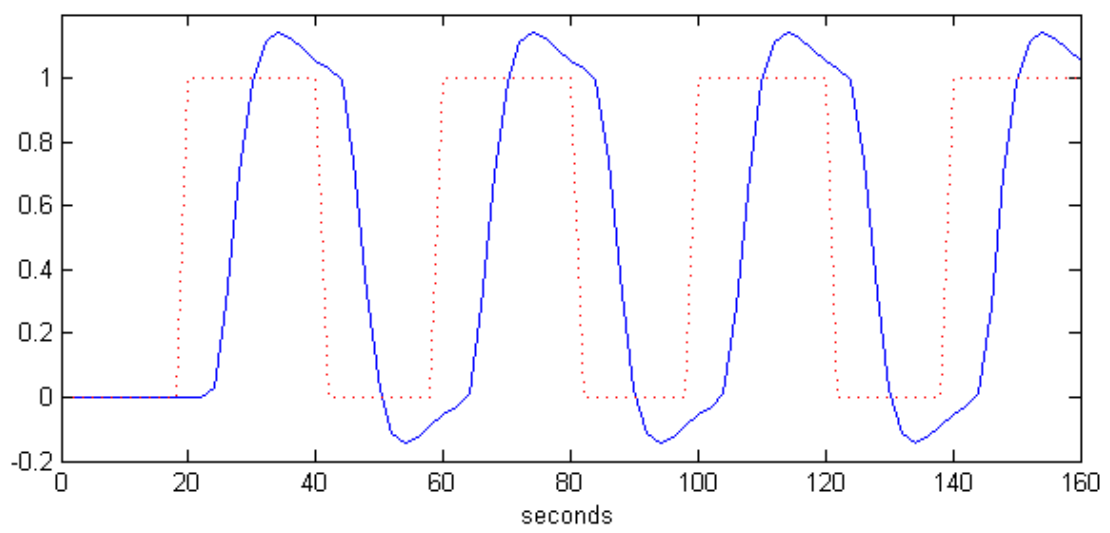

Figure 5. An ideal hemodynamic response function. The red dotted line represents the stimuli presentation time line while the blue line represents the stimuli convolved with the ideal hemodynamic response function.

The next steps in the fMRI examination process are the fMRI image acquisition and reconstruction. By means of the EPI sequence, images are acquired extremely fast, but the sequence is also prone to various artefacts that can affect the images, such as susceptibility to geometric distortions. Moreover, it is vital that the patient or volunteer does not perceptibly move the head during the minutes it takes to complete the examination. Brain activity is detected by inferring the presence of a BOLD response pattern in the voxel time series. If the subject moves his head during the acquisition, a voxel will cover different brain areas at different time points, and the possibilities to detect a BOLD response are likely to 
be deteriorated or even spoiled (in fact, when movement goes over half of the voxel size at any moment during the acquisition, e.g. a movement of $2 \mathrm{~mm}^{3}$ when the voxel is $4 \mathrm{~mm}^{3}$ size, the examination is usually discarded). Small head movements are however inevitable and they must be corrected prior to BOLD response detection by aligning the acquired EPI images. After the realignment, or motion correction, the images are usually filtered in order to improve their Signal to Noise Ratio (SNR). Realignment, filtering and other processes that improve the acquired fMRI data are known as pre-processing.

There are many available methods for the detection of active voxels. The main challenge in detecting activated voxels is the high level of noise that is present in the EPI images, and consequently also in the voxel time series, in combination with the small BOLD response effect. A straightforward detection approach is to average all images collected during the active condition and all images collected during the control/rest condition. A subtraction image should then reveal where the intensity is consistently higher during the active condition compared to the control condition. More advanced detection methods do in general apply a temporal model or reference time series to which each voxel time series is compared. The two main steps involved in the detection process are illustrated in Figure 6. For each voxel, a scalar measuring similarity between the reference time series and the voxel time series is calculated. The calculations are performed within the General Linear Model (GLM):

$$
y=\beta \cdot X+\varepsilon
$$

Where $\boldsymbol{y}$ represents the fMRI data (voxel-wise), $\boldsymbol{X}$ represents the design matrix (the expected haemodynamic response), $\beta$ is the estimation of the amplitude of $X$ and $\varepsilon$ encodes the residuals, the error, not modelled by $X$ and $\beta$. 

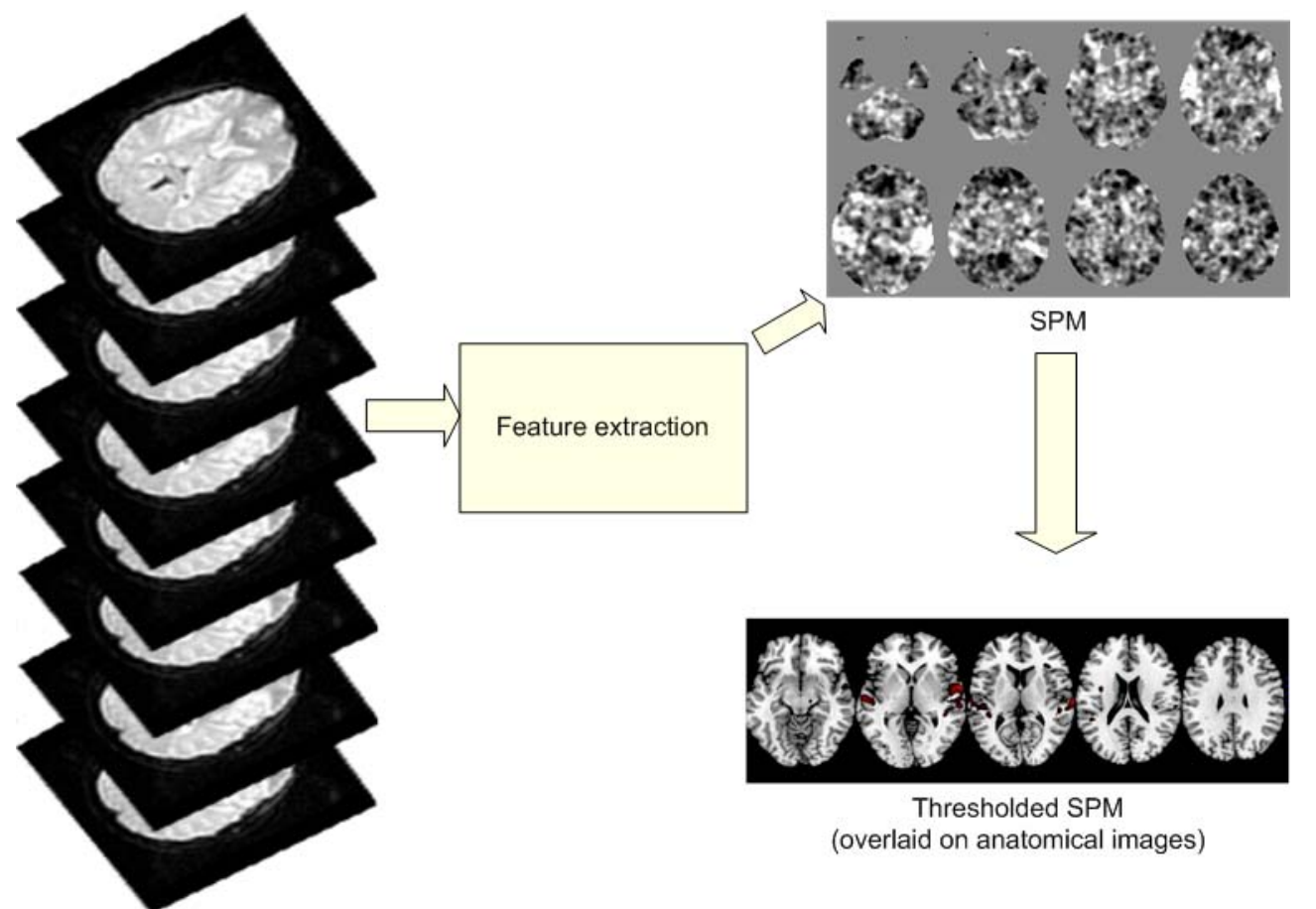

Figure 6. A Statistical Parametric Map (SPM) is obtained from the motion corrected EPI images (image stack represented on the left). Then the SPM is thresholded and most significant voxels are selected.

The result is a map of similarity measures, commonly referred to as a Statistical Parametric Map (SPM) that includes the information about the estimation of activation and the error in the measure. High similarity (high $\beta$, low $\varepsilon$ ) is interpreted as an active voxel. The SPM is for this reason thresholded in order to classify voxels as being either active or inactive, answering the question, voxel-byvoxel, of whether the null hypothesis is accepted or rejected. An extensive bibliography has been developed on how to select the correct threshold that deals appropriately with the multiple comparison problem, i.e. choosing which signals were significantly activated (see Worsley et al, 1996).

An fMRI acquisition results in a 4D spatiotemporal data set (i.e. a 3D volume, or stack of $2 \mathrm{D}$ images, which is repeatedly collected over time). Even though it is the temporal behaviour of the voxel time series that indicates brain activity, a key to a robust detection of active brain areas is to exploit the spatial nature of the data, as it has already been studied by different successful multivariate (as opposed to univariate, i.e. voxel-wise temporal course statistical 
analysis) methods, such as Independent Components Analysis (McKeown et al, 1998) and Canonical Correlation (Friman et al, 2001).

Finally, in order to draw any neurological universal conclusion it is necessary to study groups of patients or healthy subjects. Locating brain areas consistently active over the whole group is complicated by the fact that there is a high degree of anatomical and localization-of-function variability between different brains, even in healthy subjects. Currently, the most commonly adopted solution is to spatially normalize the images by means of deformations or warping so that each individual brain fits a standard brain template as well as possible. Results from different subjects can then be compared in this standard brain space.

Group studies are very common in neuroscience studies because they let us infer about the whole population, not only about the subjects of study. A similar procedure to the one shown in Figure 7 is followed.
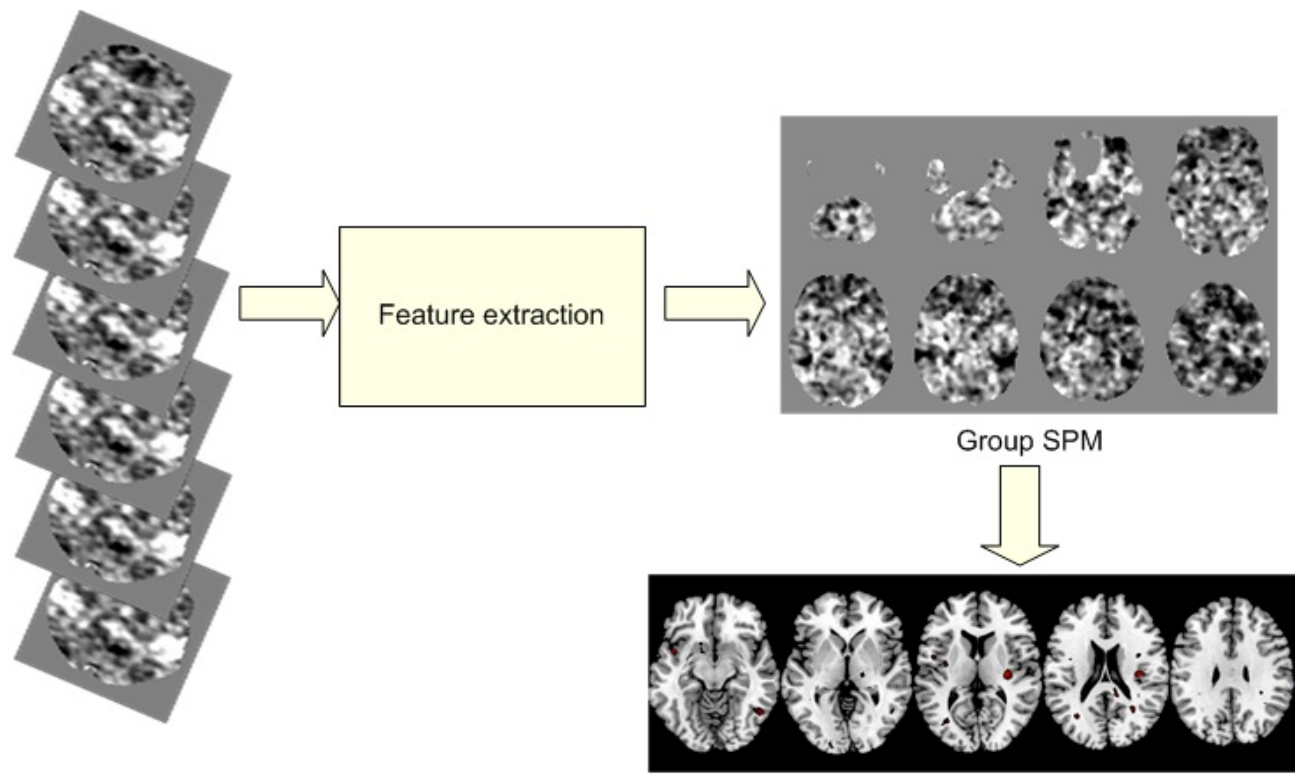

Thresholded group SPM (overlaid on anatomical images)

Figure 7. Outline of group studies. SPMs for each subject, in a normalized space, (left) are analyzed, generating a SPM representing the group. Then it is thresholded, selecting the most significant voxels from the group SPM. 
In a group study, the features extracted per subject are combined in a second level so a new statistical map is generated that summarizes all the subjects' brain activation. This is achieved by estimating $\beta$ and $\varepsilon$ in the GLM fashion, where $x$ encodes the information about the group that each data has been extracted from.

This two-step approach to the analysis (first feature-per subject extraction, then common inter-subject feature extraction) is, in a more general framework, known as Random-Effects Analysis, which is a conceptual class of the ANOVA statistical modelling.

Results are finally assessed by the experts (radiologists, neurologists, neurosurgeons, psychiatrists, psychologists...) in order to check that the experiment is correct and that the results confirm or rebut the a priori hypothesis.

\section{1.c. Schizophrenia illness research}

\section{Schizophrenia disorder}

Schizophrenia is a complex and variable, non-affective functional psychotic illness (Freeman and Garety, 2003). The age of onset is between 15 and 45 years of age, although it usually appears at the end of the teenage years.

Schizophrenia is characterized by cognitive, social and emotional impairment, and by psychotic symptoms. Psychotic (positive) symptoms refer to the loss of contact with reality, and involve delusions (fixed, false beliefs), hallucinations (aberrant, false perceptions) or bizarre behaviours. Negative symptoms are deficit states in which basic emotional and behavioural processes are diminished or absent. Typically negative symptoms are divided into affective flattening (reduced emotional expression), alogia (poverty of speech), avolition (lack of persistence), anhedonia (task completion seen as pleasureless) and apathy (Andreasen, 1982). Negative symptoms are more persistent than psychotic symptoms and are strongly related to social dysfunction. Finally, the cognitive deficits concern shortage in attention, memory and executive functions such as 
planning of behavior, set shifting ability and problem solving (see Aleman, Kahn, 2005).

A thrilling symptom in schizophrenia is auditory hallucinations as this symptom is the most common one. Hallucinations involve perceiving "('hearing') internally generated words and sentences as if they originate externally" (Tracy, Shergill, 2006). Simple hallucinations such as hearing elementary non-word sounds are not common in psychosis. Therefore, it is assumed that auditory hallucinations are an abnormality of language processing. They are also an abnormality in emotion processing: the subject attributes inappropriate emotional salience to sensory stimuli in the environment, either real or imagined (Laviollete, 2007).

\section{Schizophrenia. Functional Neuroimage}

With the advent of fMRI, functional neuroimaging studies about schizophrenia have increased dramatically (see Figure 8). Determining which areas in the brain have an abnormal functioning may help in the treatment of schizophrenia.

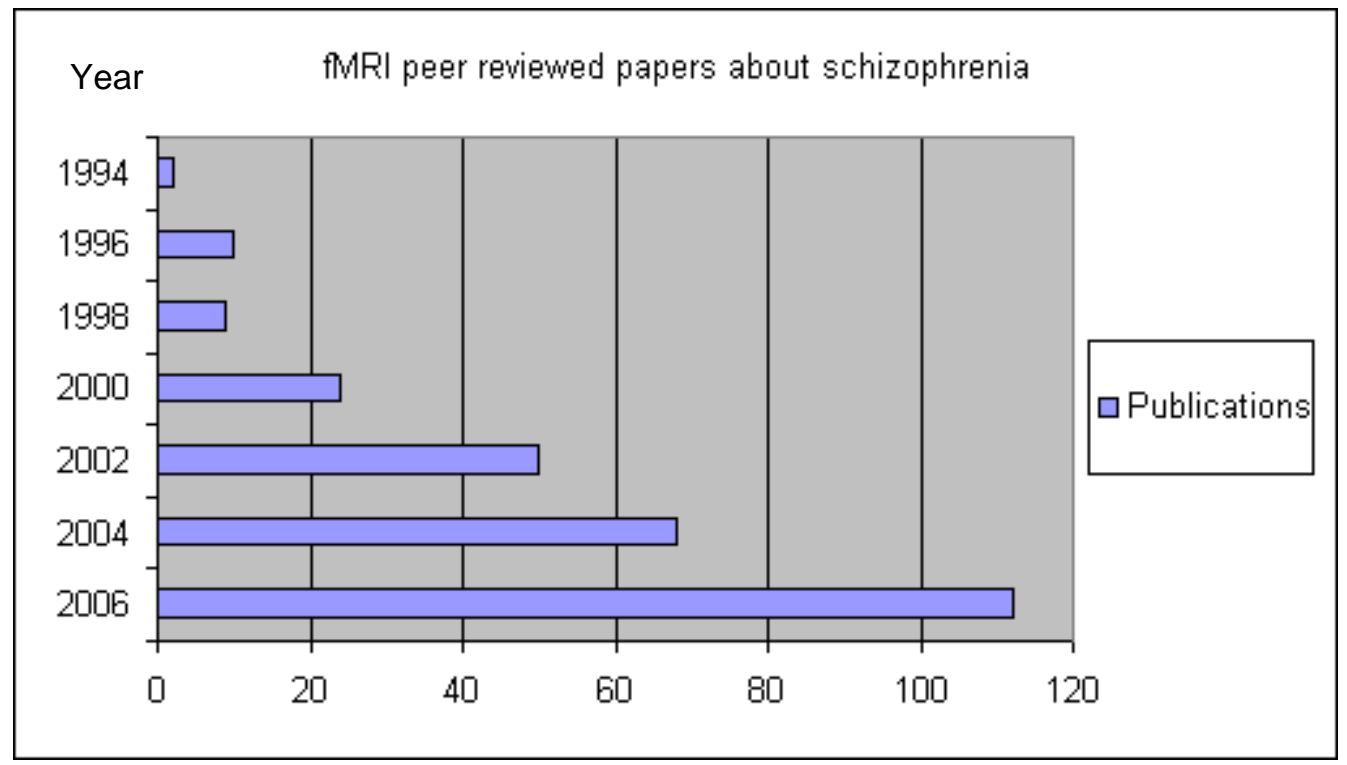

Figure 8. PubMed manuscripts about fMRI and schizophrenia. Search performed with the sentence: ("fMRI" or "functional MRI") and schizophrenia. 
In recent years, there have been multiple experimental paradigms that have studied the activity in the healthy brain, with very different complexity. These have been divided according to the stimulus modality (motor or sensorial) or the type of cognitive task to be studied. The analysis of observable brain activation according to an emotional content stimulus has generated great interest. Because of the importance of emotional processing in schizophrenia, those studies are very noticeable in this context. In an extensive revision on the subject, Murphy and colleagues (Murphy et al, 2003) analyzed 106 neuroimaging studies that had focused on analyzing the differences on brain activation observed between emotional paradigms versus non-emotional ones in healthy subjects. Almost $90 \%$ (95 papers) of these studies used the visual stimulus modality, mainly with presentation of emotional expressions in faces or of emotional content images, the remaining experiments being distributed between auditory (11 studies), olfactory (5 studies), tactile (2 studies), gustatory (2 studies) stimuli and even electric shock and saline injection (1 study each). The scarce number of studies with auditory modality is striking, especially considering the great importance of language and word processing in the priming of human emotions.

In very general terms, some authors have stated that all the psychiatric disorders are tightly tied to alterations in the emotional activation (Lynam, Widiger, 2007). Thus, the large amount of studies that use an emotional paradigm in subjects with psychiatric diseases (Philips, 2003) is hardly surprising. Focusing on schizophrenia, many studies have demonstrated deficits in the emotional processing in recognition of faces and in affective prosody. To the PhD candidate's knowledge, no neuroimaging fMRI study that used the auditory modality in schizophrenic subjects with auditory hallucinations, that could be generalized to the whole population, existed in the literature prior to the ones presented in this dissertation (as according to the search for "auditory "fmri" emotion schizophrenia" in PubMed). 


\section{Thesis motivation and objectives}

"A handful of patience is worth more than a pound of brains." -Dutch Proverb

\section{2.a. - Assumptions}

Concerning the BOLD response and the fMRI data, after a bibliographical review, the main assumptions used in this thesis are:

The first assumption is that the BOLD response can be modelled as a linear system, i.e. the BOLD response evoked by two stimuli applied close in time can be superimposed or linearly added. The linearity assumption seems to hold reasonably well in most situations (Boynton et al., 1996) and the convolutive model is therefore frequently used in fMRI analysis, even though deviations from linearity have been reported in special cases (Vazquez, Noll, 1998; Birn et al., 2001).

When considering only one voxel timecourse at a time, all spatial dependencies that exist in an MR image are ignored (Zarahn et al, 1997). Sometimes spatial smoothing is applied to the fMRI data prior to statistical analysis, as a remedy to reduce noise and to introduce information from other voxels in the one considered. Spatial smoothing of the fMRI data is also needed when Random Field Theory is applied (Worsley et al, 1996). Hence, spatial smoothing has been kept as the mainly used filtering technique for fMRI data. There are different problems in the interpretation of spatial smoothed results, which have been lately described (Reimold et al, 2006; Walker et al, 2006; Wink AM, Roerdink, 2004). The second assumption is that the precision in the spatial localization of activation areas is reduced by the spatial smoothing of the data. 
Concerning schizophrenia illness and the experimental study, the main assumptions used along this study are:

The first assumption is that BOLD response can be actually studied in schizophrenic patients and it can be directly compared to the BOLD response in healthy subjects.

The second assumption is that subjects have been collaborative, in the sense of having paid attention to the stimuli. Therefore, the statistical maps are an approximate representation of the subjects' response to the stimuli.

A deeper comprehension of the schizophrenia illness can be obtained by means of the study of the brain (function, morphometry, brain function-related genes ...). Schizophrenia is a poorly understood illness because many different symptoms have been attributed to schizophrenia. The third assumption is that a specific symptom, such as predisposition to auditory hallucinations, should be selected and used as a biomarker to study brain function in schizophrenia illness.

The fourth assumption is that auditory stimulation can be a more direct way to replicate the emotional response to auditory hallucinations in schizophrenic subjects than other stimulation paths, such as the visual or the olfactory paths, as the most specific positive symptom in schizophrenia is the presence of auditory hallucinations. 


\section{2.b - Hypothesis}

The first hypothesis is that auditory verbal stimulation, both with emotional and neutral content, applied in an fMRI paradigm, will let us observe the brain activation due to auditory semantic emotional processing. Furthermore, it will let us observe the areas of activation separately in three groups of subjects: a) healthy subjects, b) schizophrenic patients with auditory hallucinations and c) schizophrenic patients without auditory hallucinations.

The second hypothesis is that a novel filtering technique, the NL-Means filter, can enhance the localization of the fMRI BOLD-related response present in the data without appreciable resolution loss.

\section{2.c. - Thesis contributions}

- The study of brain activation in schizophrenia with a semantic emotional auditory paradigm has been developed and carried out. New knowledge about emotional response in the brain with schizophrenia has been achieved.

- Activation has been studied and described: a) in healthy subjects and $b$ ) schizophrenic patients with and without auditory hallucinations.

- A method that joins the areas found in a) structural MR volumetric analysis of areas with gray matter loss in schizophrenic patients with respect to controls; and in b) differences in fMRI activation between schizophrenic patients and controls.

- General fMRI software phantom implementations are introduced.

- The first application and adaptation of the NL-Means algorithm to fMRI data is achieved. An optimization of the original NL-Means is introduced. The NL-Means method has been applied to the acquired fMRI data from the three study groups. 


\section{2.d. - Thesis objectives}

1. To study brain activation in schizophrenia with a semantic emotional auditory paradigm. This study comprises the development of the paradigm and the study of emotional response in the brain with schizophrenia.

2. To study and compare brain activation, with the paradigm stated above: a) in healthy subjects and $b$ ) in schizophrenic patients with and without auditory hallucinations.

3. To develop a method to determine coincidence areas of two effects: a) areas with gray matter loss in schizophrenic patients compared to controls and $b$ ) areas with $\mathrm{fMRI}$ brain activation differences in response to the semantic emotional auditory paradigm between schizophrenic patients and controls.

4. To adapt and to apply the NL-Means algorithm to fMRI data with the aim of removing noise without significant resolution loss, with the intention of improving results in the localization in the schizophrenia research project.

\section{2.d. - Thesis outline}

This dissertation presents a unique study of schizophrenia illness. Areas of the brain that are activated because of auditory emotional semantic processing are shown. It also presents the NL-Means filter and its application to fMRI, specifically to the fMRI study of schizophrenia auditory hallucinations. In chapter $\mathbf{3}$, the experimental paradigm, that will be the base for the study of schizophrenia that has been carried out, is presented.

Chapter 4 evinces the study of the emotional response in a very homogeneous sample of schizophrenic patients with auditory hallucinations. The brain activation estimated by means of fMRI in schizophrenic subjects is compared to the one estimated in the healthy subjects population. 
Chapter 5 presents the areas that coincide in the impaired functioning brain (measured by means of fMRI) and the impaired structural brain. Coincidence areas are shown stereotactically. These areas are very probably involved in the prognosis and diagnoisis of schizophrenia as they appear damaged both in their function and structure.

Chapter 6 shows the application of the NL-Means filtering method to fMRI data, its implementation and optimization. It is also applied to the schizophrenia fMRI data, and its advantages are shown. The results are then compared to the ones obtained in the previous chapters so localization and signal amount estimation are reassessed with the data re-processed with the proposed filter.

Chapter 7 states the main results, the conclusion and future lines of research. Specifically, it suggests what should be done next, after the initial results with NL-Means applied to fMRI have been shown, in order to take the maximum advantage of the filtering technique. Different approaches are presented that could make the algorithm faster and that would also increase its accuracy. Application of the filtering technique to the research of schizophrenia is also posed. 


\section{3. Emotional auditory paradigm in neuroimaging: a base for the study of psychosis}

"Great thoughts come from the heart" - Luc de Clapiers (1715-1747), Réflexions et maximes, 1746.

The fMRI experimental paradigm that has been implemented for the schizophrenia research project is presented in this chapter.

The aim is focused on the implementation and analysis of a paradigm that can be used in fMRI for detecting the areas of the brain that activate due to auditory emotional semantic processing and the areas that activate due to auditory neutral semantic processing.

In the developed paradigm, there were two stimulation types, neutral and emotional, which were presented by the auditory path in a semantic processing task. Thus, at least detection of activation in the superior temporal lobe was expected (Binder et al, 2000) as a marker of successful acquisition.

For this study, the fMRI acquisition sequence needed the initial optimization of the acquisition parameters. Bibliography research and experimental studies were therefore performed. Parameter optimization was achieved in two steps: First, a simple motor (hand movement) paradigm was developed and tested in two 1.5T scanners, with final parameters for successful fMRI data acquisition obtained: 24 slices with a GRE-EPI, TR=2000 ms, TE=50 ms, voxel size=1.72 x $1.72 \times 5 \mathrm{~mm}, 96 \times 96$ acquisition matrix, FOV=220 mm, no inter-slice gap (Lull et al, 2004). Then, the finally implemented auditory paradigm was tested. 


\section{3.a. - Quality assessment of the acquired data}

Although not very commonly explained in the manuscripts, quality assessment of the acquired fMRI data is necessary before applying corrections to the images and statistics. There are different ways to check for common artefacts and excessive movement in the data. One way that is straightforward is manual observation of the fMRI data. Most acquisition problems such as the spin-history effect, excessive inhomogeneities or data transfer errors can be detected visually.

Furthermore, summary images and plots can be generated that show possible outliers. As an example, Figure 9 shows a plot of the mean intensity per each volume from a subject's fMRI data, within the schizophrenia research project. In the plot a dropout of intensity can be seen in the volume 39 that should be considered.

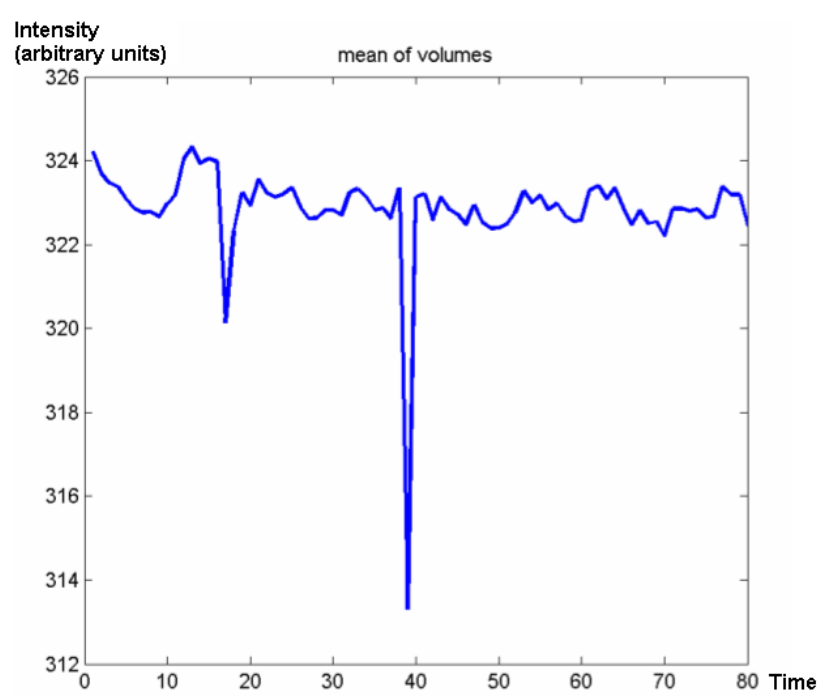

Figure 9. Plot of the mean intensity value for every volume in each temporal position. As observable, there is a big change in the mean intensity of the volume 39. 
Another example is shown in Figure 10. K-space acquisition problems are present in one of the acquired volumes. In this case, careful inspection was necessary to detect the artefact, as it was present in some slices only. Therefore, an inspection of all temporal positions in one slice alone could have led to undetection of the artefact.
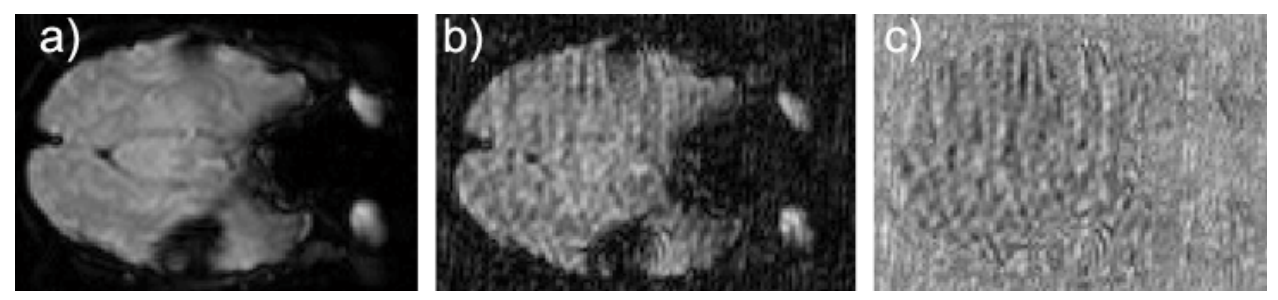

Figure 10. Two contiguous volumes from an fMRI sequence, a) and b). c) Shows the subtraction between a) and b). K-space acquisition problems are present in $b$ ).

Figure 9 and Figure 10 show the importance of having a quality assessment technique for the acquired fMRI data, such as the one developed by the $\mathrm{PhD}$ candidate in direction of the Leonardo Da Vinci student Antonios Antonious, AQUA (IBIME Research Group, Valencia, Spain; the tool is accessible via http://www.ibime.upv.es/mi).

\section{3.b. - Data analysis}

The analysis included the pre-processing of the fMRI data for each subject, incorporating realignment of the data, registration of the high-resolution T1 volume to the EPI fMRI data and spatial normalization of the fMRI data to a standard template. These steps can be followed through the SPM interface, as shown in Figure 11. 


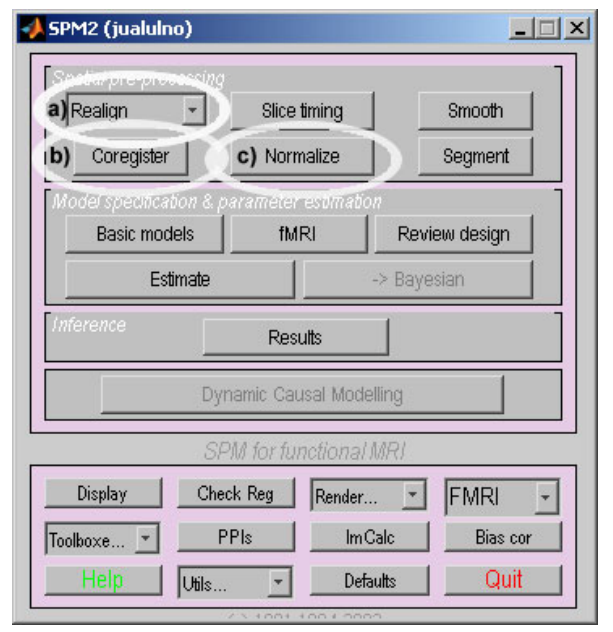

Figure 11. Part of SPM2 interface, with a) realignment, b) coregistration and c) normalization buttons highlighted. a), b) and c) are the interface to the basic preprocessing of the fMRI data.

The realignment (i.e. movement correction) step, crucial to any fMRI activation study (Oakes et al, 2005), generated a mean volume of the fMRI data that was used as the source volume image for the co-registration. Co-registration is the process that matches two volumetric images that are possibly of different type because they have different acquisition parameters; they can also be from different subjects (Ashburner et al, 1997).

Spatial normalization was finally applied. The spatial normalization consisted of linear and nonlinear (i.e. warping) spatial transformations (Ardekani et al, 2005; Friston et al, 1995; Woods et al, 1998) that matched each subject's anatomical volumetric image to a standard template. The last pre-processing step consisted of spatially smoothing the fMRI data, as "spatial smoothing increases the relative power of low frequencies (including the assumed paradigm frequency)" (Aguirre et al, 1997).

Statistics were performed by means of the General Linear Model (GLM), in a two-level analysis (also known as Random Effect Analysis). Firstly, in a GLM individual fMRI data are analyzed and parametric volume images are obtained by fitting the data to a linear model. Secondly, the parametric volume images from 
each subject are taken to a group statistical test that will allow the detection of common patterns of activation in a group or differential patterns of activation between groups (Frackowiak et al, 2003).

\section{3.c. - General Linear Model}

As introduced in Chapter 1, the detection of activated voxels is carried out by means of statistical tests. Linear statistics (e.g. T-tests, ANOVA) can be performed in a GLM.

A Linear Model explains the response variable $y$ of length / in terms of a linear combination of explanatory variables plus an error term:

$$
y=X \cdot \beta+\varepsilon
$$

In this GLM, $\boldsymbol{X}$ is a matrix that contains $j$ explanatory variables of length $I$. $\boldsymbol{\varepsilon}$ is the error term and $\beta$ is a vector with $j$ length that encodes the unknown variables.

Linear statistic tests can be included in a GLM. The simplest case of a linear regression could be stated as:

$$
Y=\mu+X \cdot \beta+\varepsilon
$$

Where the unknown parameters are: $\mu$, a constant term in the model, the regression slope $\beta$ and the error term $\varepsilon \sim N\left(0, \sigma^{2}\right)$.

A GLM could be stated that was identical to the linear regression:

$$
Y=X \cdot(\beta+\mu)+\varepsilon
$$

In equation (3) the difference with equation (2) is that there is a new variable that has been encoded into $\boldsymbol{X}$, which we set to 1 for all its values; furthermore, the notation has been changed to matrix notation (upper case bold indicates matrix while lower case bold indicates vector). Thus, the new column is a dummy variable that lets us state the linear regression as a GLM. In a similar 
fashion we can perform different T-tests and Factorial (i.e. ANOVA) designs as GLM models.

A SPM is the estimation of a combination of some parameters $\beta$ for each voxel. The usual graphical representation shows the estimation for each voxel coding the statistical values into colour values, as observable in Figure 12.
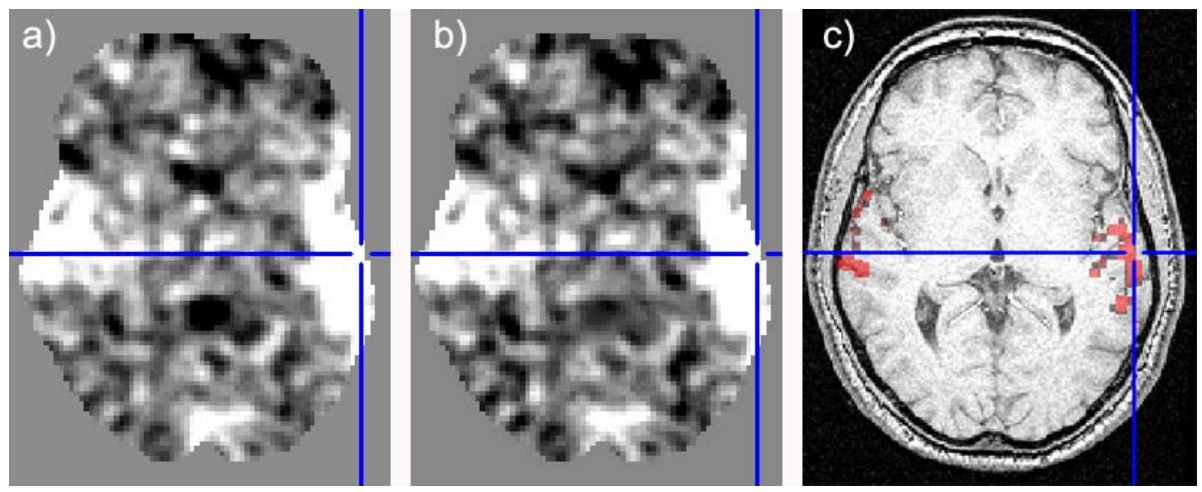

Figure 12. Results for a GLM design from real fMRI data, showing colour-coded parameters. Crosshairs mark the location of the highest value in a) a $\beta$ map, b) the corresponding $\beta$-divided-by-error map (SPM map) and c) the SPM corresponding to high values from b), overlaid on the subject's anatomical image.

In an fMRI study, $X$ and $Y$ are known. $Y$ represents the fMRI data. The values in $\boldsymbol{X}$ are the design matrix, usually represented colour-coded. As an example, we can have an fMRI study corresponding to a subject that has had two identical blocked-design fMRI sessions. The hemodynamic signal that we expect in one session would have a similar shape to the one seen in Figure 5 (Chapter 1). That haemodynamic signal corresponds to Figure 13,b), first column. In Figure $\underline{13, b)}$ two fMRI sessions have been encoded in the first two columns and the last two columns account for inter-session variability. The first rows of b) can be observed numerically in Figure 13, a). 


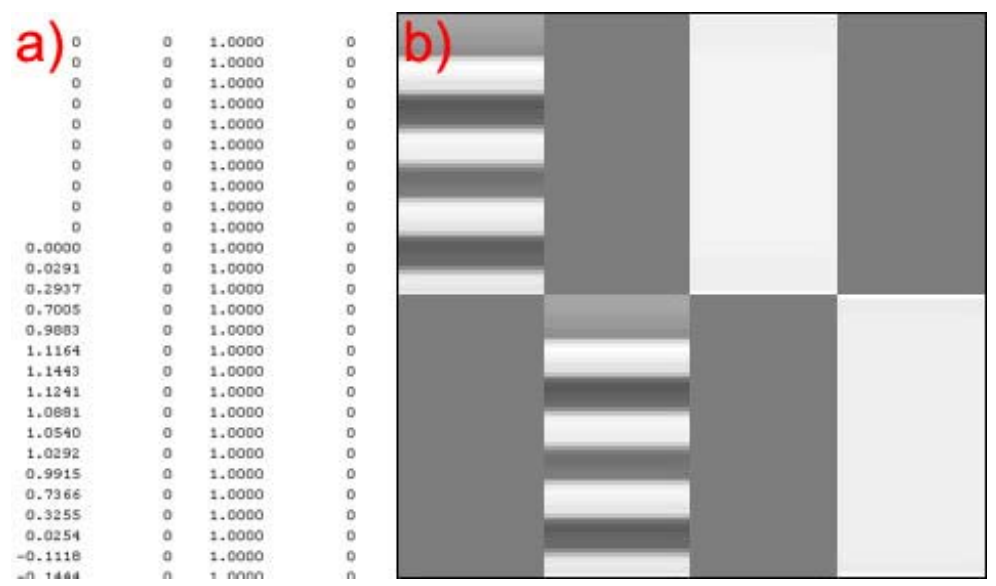

Figure 13. fMRI Design matrix. a) Design matrix values and b) The same design matrix, colour-coded (gray values). Values in $\boldsymbol{X}$ needed in a specific GLM are partly listed in a) and drawn in b).

After having defined $Y$ and $X$, the unknown variables ( $\beta$ parameters vector) have to be calculated. This can be done by estimating the parameters that best fit the data. The calculation of the $\beta$ parameter vector has traditionally been done by means of a least squares fit. Nowadays models introduce corrections for non-normality of the error terms (e.g. drift terms in the fMRI, serial correlation in the data) that enlarge the least squares estimation by including deviations from normality such as serial correlations or drift terms, implicitly in the GLM.

Finally, the SPM map is usually thresholded and therefore highest significant voxels are selected.

During the presented work, temporal characteristics of the BOLD signal were investigated (Buxton et al, 1998; Logothetis et al, 2001). Time to peak (time from the presentation of the stimulus to maximum hemodynamic change) is estimated to be around $4.7 \mathrm{~s}$ but the time to peak is variable among the normal population, the brain area that is involved in a task, age, etc. so a detailed study must be done to select the length of stimuli. 


\section{3.d. - Multiple comparison testing}

As the approach that has been followed in the presented work is a univariate approach (timecourse of each spatial voxel is analyzed independently), statistical values must be corrected so that multiplicity is taken into account. A variety of multiple comparison correction techniques have been proposed in the neuroimaging field. The most straight-forward is Bonferroni correction that corrects for multiple independent comparisons. The Bonferroni correction, in this context, states that if there are $\mathrm{n}$ voxels (therefore $\mathrm{n}$ statistical comparisons), the corrected $p$-value should be:

$$
\alpha_{B F}=\frac{\alpha}{n}
$$

Where $\alpha$ is the desired statistical threshold (p-value) and $\alpha_{B F}$ is the corrected statistical threshold. As an example, if we want a $p<0.05$ and there are 300.000 voxels, the corrected threshold would be: $\alpha_{B F}=\frac{0.05}{300.000} \approx 1.67 \mathrm{e}^{-7}$.

However, Bonferroni correction is too conservative, mainly for two reasons. Firstly, the method is valid when the multiple comparisons are independent. Secondly, the correction deals with the probability that no voxel is estimated as activated by chance, i.e. that no false positives exist (instead of dealing with e.g. the proportion of false positives). Therefore a proportion of activated voxels will be estimated as not activated (false negatives) (Perneger, 1998).

Both deficiencies in Bonferroni correction have been reviewed and new methods have been proposed for correcting for multiple comparison testing.

The Family Wise Error (FWE) correction by means of estimating the Euler Characteristic deals with the first problem of Bonferroni correction. fMRI statistical tests are not independent. Activation does not appear spatially isolated in fMRI data. Furthermore, data can be seen as a set of Gaussian Random Fields (Worsley et al, 1992). Therefore, we could see an fMRI volumetric image as a 
number of independent smooth clusters and the correction could be applied to the number of clusters instead of voxels. The independent smooth clusters that are present in an image are named Resolution Elements or 'RESELS' by Worsley (Worsley et al, 1992). If an image can be seen as a smooth random field, then the image will be composed of a number of such fields (see Chapter 14, Frackowiak et al, 2003). Figure 14 shows a random image and the same image that has been smoothed, leaving 128 resolution elements (b), from the original independent $128 \mathrm{x}$ 128 voxels (a).
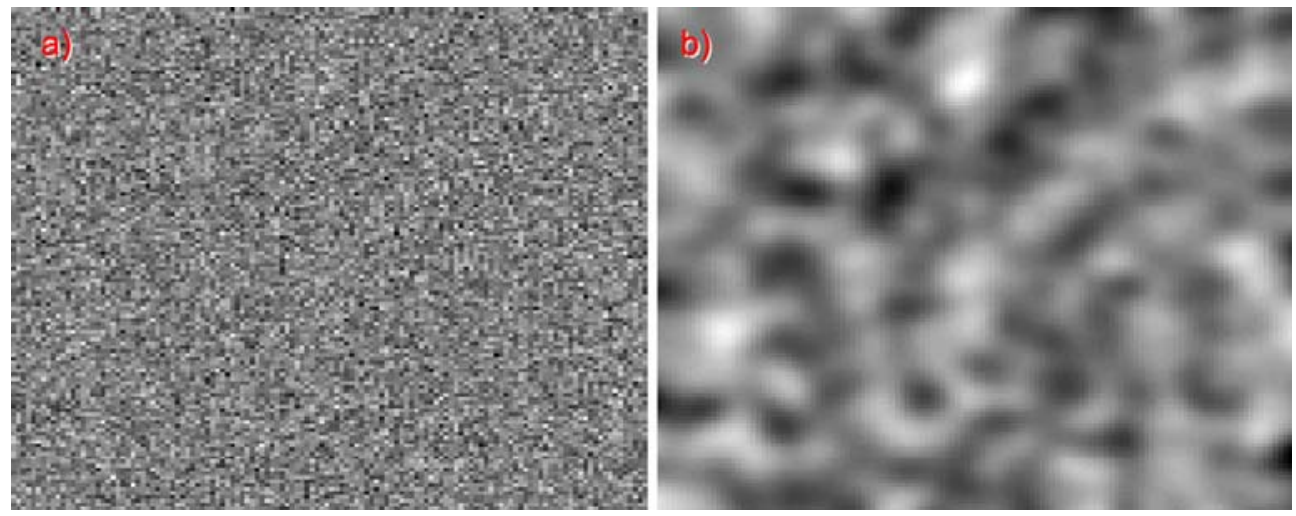

Figure 14. a) Uniformly random generated image with $128 \times 128$ pixels. b) Image from a), smoothed with a Gaussian kernel of FWHM 8 by 8 pixels.

$$
\alpha_{F W E}=\frac{\alpha}{n_{\text {RESELS }}}
$$

Equation (5) states the basic idea of FWE with the Euler Characteristic (for a deep insight on the FWE technique, see Nichols, Hayasaka, 2003). The number of resels in an image is estimated by means of the Euler Characteristic (Worsley et al, 1992).

A method that deals with the proportion of false positives instead of dealing with the chance of any false positive is False Discovery Rate (FDR, Benjamini, Hochberg, 1995). The FDR method was adapted to functional neuroimaging by Genovese in 2002 (Genovese et al, 2002). The concept remains the same as it was presented by Benjamini in 1995. In a typical fMRI experiment, thousands of 
tests are performed and a threshold must be chosen that rejects the null hypothesis (i.e. voxels that are estimated as active). Selecting a threshold that takes into account how many false positives are introduced per each voxel estimated as active is more intuitive than controlling the probability of making one or more false positive over all hypothesis tests. Setting a rate of false positives per true positives has other advantages over FWE methods. The main advantage is that, compared to the other known methods, the error rate that is introduced in our estimation of voxels above the threshold remains always the same. However, any single choice of threshold (as opposed to rate) across data sets will result in an error rate that is too high for some data and too low for others.

\section{3.e. - Implementation of the data analysis}

The fMRI data was an auditory paradigm, including two sessions. Both sessions included stimuli distributed in a block design. The data were realigned, co-registered and spatially normalized. Afterwards, a design matrix was generated that included the GLM model for detecting which voxels were activated. The blocks of stimulation were distributed in an ON-OFF paradigm, as observable in Figure 5 (Chapter 1). The ON level was achieved by words presented auditorily. The OFF level was achieved by silence. The subjects listened to the words passively, without emitting any judgment about the words. There were two sessions and stimuli were different in both sessions. The first session included neutral words as stimuli while the second session included high emotional words as stimuli. A design matrix that included both sessions was generated, taking into account the haemodynamic model (Chapter 11, Frackowiak et al, 2003), identical to the one represented in Figure 13.

The beta parameters from the experiment were extracted for each individual subject in a Restricted Maximum Likelihood scheme (ReML). Maximum Likelihood (ML) estimation is a method that fits linear models assuming independent, identically distributed data. However, this is not the case in fMRI data, as temporal correlations exist in the data due to the persistence of neuronal activation, cyclical events (such as aliased cardiac and respiratory cycles), or other characteristics or artifacts of the measurement process, amongst others (Locascio 
et al, 1997). ReML, as opposed to ML estimation, can produce unbiased estimates of variance and covariance components. The ReML estimates can then be used to whiten -removing the temporal correlation present in- the data giving $\mathrm{ML}$ estimators. Different methods have been proposed to whiten fMRI data. Locascio et al (1997) proposed to use a model based on the autoregressive moving average model. Zarahn et al (1997) observed $1 / f$ noise profiles in fMRI data and therefore used a $1 / f$ noise model to treat temporal correlations. More recently, Woolrich proposed the use of a Tukey taper, windowing the raw autocorrelation estimate, after high-pass filtering the data (Woolrich et al, 2001). In the current study, serial correlations were treated by incorporating an Autoregressive system (Box et al, 1994) with one autoregressive term set to 0.2. Error covariance constraints were extracted and incorporated into the estimation of the beta parameters.

A statistical parametric map was generated for each condition (i.e. a SPM for $\beta_{1}$ that modeled the brain activation when neutral auditory stimuli were presented and $\beta_{2}$ that modeled the brain activation when high emotional content words were presented as stimuli).

FDR multiple comparison testing correction was selected as appropriate for the presented study as it enables the detection of signals present in the data without the introduction of any activation detection if there is no signal in the data.

\section{3.f. - SPM software}

Most of the work was developed based on a learning process about the use of the Statistical Parametric Mapping (SPM) method for detecting brain activity from neuroimaging data (Frackowiak et al, 2003). A free software tool that is used to a great extent in the neuroimaging community, also named SPM (FIL, Wellcome Trust Centre for Neuroimaging, London, UK), has also been very important. This training has been useful to different funded projects that have been carried out by the research group. It has also been useful in the application of the method to clinical cases. In the learning process, a significant tool has been the SPM mail list, where different experts in the neuroimaging field, such as John Ashburner, Karl Friston, William Penny, Klaas E. Stephan and Keith Worsley discuss and explain different questions, from mainly technical to state-of-the-art ones. The SPM mail list is an obliged source of information for the scientific community that researches 
about functional neuroimaging, although the list is mainly focused on the SPM software package.

\section{3.g. - PhD candidate contributions}

The tasks carried out by the PhD candidate were:

- Collaboration in the development of the paradigm, especially taking into account time restrictions necessary for optimal BOLD signal detection.

- Collaboration in the estimation of the best parameters for the fMRI sequence.

- $\quad$ Initial assessment of brain activity detection from the fMRI data.

- $\quad$ Preparation of the fMRI data (also known as pre-processing). This included image quality assurance and movement assessment while in the fMRI scanner. The pre-processing also incorporated movement correction, co-registration to the subject's anatomical images and normalization to the standard brain template.

First level (subject-wise) and second-level (multi-subject) statistical analyses following the General Linear Model in a voxel-by-voxel (univariate) basis.

Generation of figures two and three in the article (Figures 15 and $\underline{16}$ at the end of this chapter).

- Collaboration in editing the manuscript, mainly the Methods and Results sections.

A fMRI data quality assessment toolbox was directed by the candidate and developed by the Leonardo Da Vinci student Antonios Antoniou. The toolbox has been published at the website: http://www.fil.ion.ucl.ac.uk/spm/ext. 


\section{Emotional auditory paradigm in neuroimaging: a base for the study of psychosis}

Julio Sanjuán, Juan J. Lull, Luis Martí-Bonmatí, Eduardo J. Aguilar, Marien Gadea, David Moratal-Pérez, José C González, Montserrat Robles.

Actas Esp Psiquiatr 2005. 33(6):383-9 
J. Sanjuán ${ }^{1}$

J. J. Lull ${ }^{2}$

L. Marti-Bonmati ${ }^{3}$

E. J. Aguilar ${ }^{4}$

M. Gadea ${ }^{5}$

D. Moratal-Pérez ${ }^{2}$

J. C. González ${ }^{1}$

M. Robles ${ }^{2}$

\section{Emotional auditory paradigm in neuroimaging: a base for the study of psychosis}

\begin{tabular}{|c|c|c|}
\hline${ }^{1}$ Medicine Department & ${ }^{3}$ Radiology Service & ${ }^{5}$ Psychobiology Area \\
\hline Psychiatry Unit & Hospital Universitario Dr. Peset & Psychology School \\
\hline Universidad de Valencia. Spain & Valencia. Spain & Universitad de València \\
\hline $\begin{array}{l}2 \text { Bioengineering, Electronic and } \\
\text { Telemedicine Group } \\
\text { Universidad Politécnica de Valencia. Spain }\end{array}$ & $\begin{array}{l}{ }^{4} \text { Psychiatry Service } \\
\text { Hospital Sagunto } \\
\text { Valencia. Spain }\end{array}$ & Valencia. Spain \\
\hline
\end{tabular}

Introduction. Since the arrival of neuroimaging numerous studies have tried to analyze the differences between emotional and non-emotional response. The majority of these studies use visual approach (faces) and begin with data in normal subjects. The present study introduces a new paradigm for the study of emotional response based on auditory approach and designed specifically for the study of psychoses.

Method. The most frequent words heard by psychotic patients with auditory hallucinations were analyzed. They were classified according to five categories which were compared with 13 other words with the same structure but with a neutral emotional valency. This paradigm was applied to see the cerebral activation with functional magnetic resonance imaging (fMRI) in 10 right handed healthy males.

Results. In the preliminary analysis a clear differentiation is observed depending on the type of stimulus applied (emotional or non-emotional), both in the intensity of activation (right and left temporal cortex) as in the activation of specific areas (right precentral and supramarginal gyrus) only with the emotional stimulus.

Conclusions. The present paradigm allows the observation of a differentiation in the cerebral activation to emotional auditory stimulus and could be of utility in the study of psychotic patients.

Key words:

fMRI. Auditory paradigm. Emotional processing. Words.

Actas Esp Psiquiatr 2005;33(0):00-00

\section{Paradigma auditivo emocional en neuroimagen: una base para el estudio de la psicosis}

Introducción. Desde la llegada de la neuroimagen numerosos estudios han tratado de analizar las diferen-

$$
\begin{aligned}
& \text { Correspondence: } \\
& \text { Julio Sanjuán } \\
& \text { Unidad Psiquiatria } \\
& \text { Departamento de Medicina } \\
& \text { Universidad de Valencia } \\
& \text { Av. Blasco Ibáñez, } 15 \\
& \text { 46010 Valencia. Spain } \\
& \text { E-mail: julio.sanjuan@uv.es }
\end{aligned}
$$

cias en la respuesta emocional frente a la no emocional. La mayoría de estos estudios utilizan la modalidad visual (caras) y parten de los datos en sujetos normales. En el presente estudio se presenta un nuevo paradigma para el estudio de la respuesta emocional basado en la modalidad auditiva y diseñado específicamente para el estudio de la psicosis.

Método. Se analizaron las palabras más frecuentes que oían los pacientes psicóticos con alucinaciones auditivas, se clasificaron según cinco categorías y a partir de las mismas se diseñó un tren de 13 palabras emocionales, comparándose con 13 palabras con la misma complejidad sintáctica y con una valencia emocional neutral. Se aplicó este paradigma para ver la activación cerebral mediante resonancia magnética funcional (RMNf) en 10 varones sanos y diestros.

Resultados. En los análisis preliminares se observa una clara diferenciación según el estímulo sea emocional o no emocional, tanto en la intensidad de la activación (córtex temporal derecho e izquierdo) como en la activación de áreas específicas (precentral y supramarginal derecha).

Conclusiones. El paradigma presentado permite observar una diferenciación en la activación cerebral de la respuesta a estímulos auditivos emocionales y podría ser de utilidad en pacientes psicóticos.

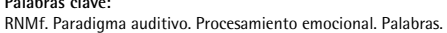

\section{INTRODUCTION}

Since the studies of Charles Darwin on the expression of emotions in man and animals ${ }^{1}$, many investigators have tried to analyze the biological bases of our emotional response, both from animal research ${ }^{2}$ and from the search for biological correlates to the different emotions ${ }^{3}$. Classic theories tried to relate the peripheral changes to stimuli of different emotional content to establish a psychophysiology of the emotions. The arrival of functional neuroimaging through magnetic resonance (fMRI) has opened a new and exciting perspective in this field. 
Functional neuroimaging studies can be divided into three sections: those that seek differences in the baseline condition of the subject without subjecting him/her to any stimuli, those that try to capture a specific and special moment of the brain functional activity (as, for example, pressing a button when the subject experiences hallucinations) and those others that try to measure brain functional activity while the subject undergoes a type of stimulus or task.

Thus, within functional neuroimaging, "paradigm" is called the stimulus or task of experimental design in which the subject is repeatedly subjected to two or more situations. During recent years, there have been multiple paradigms for the study of brain activity with very different complexity. These have been divided according to the stimulus modality (motor or sensorial) or the type of cognitive task to be studied. For the extraction of results, these situations normally include the performance of one or more specific functional tasks and the baseline rest situation ${ }^{4}$.

However, the analysis of the differences observed in brain activation according to whether the stimulus has an emotional content or is emotionally neutral is one of the areas that generates the greatest interest. In an extensive revision on the subject, Murphy ${ }^{5}$ analyzed 106 neuroimaging studies that had focused on analyzing the differences on brain activation observed between emotional paradigms versus non-emotional ones. Almost $90 \%$ (95 papers) of these studies have used the visual stimulus modality, mainly with presentation of emotional expressions in faces or of emotional content images ${ }^{6-9}$, the remaining experiments being distributed in auditory (11 studies), olfactory (5 studies), tactile (2 studies), gustatory (2 studies) stimuli and even electric shock and saline injection (1 study each).

The scarce number of studies with auditory modality is striking, above all if we admit the great importance of language and the word in awaking human emotions. The few studies that use an auditory paradigm with emotional response in normal subjects appear in table 1 . As can be observed in this table, some studies have sought to provoke different emotions with music ${ }^{10-12}$, or noise ${ }^{13-15}$ while others have used the presentation of words having different emotional meaning ${ }^{10-26}$. The paradigms used in each one of these studies have been very different and thus it is complicated to compare the results. Some studies have sought to analyze the capacity of identification of the emotional tone (prosody) ${ }^{16-19}$ while others have studied the differentiation in the activation pattern according to the type of emotion related with the word ${ }^{20-26}$. In keeping with Maddock $^{24}$, the variables that influence the fact that a word may arose an emotion is mainly associated with the emotional value given to it by the subject, to the frequency of its use and to the imagination it can evoke. The complexity (number of syllables) of the word is another factor to consider. These authors found activation in the upper part of the left cingulate cortex in a sample of eigth right handed subjects (six women and two men), without being able to observe differences between pleasant stimuli versus unpleasant ones.

All these investigations have great relevance for the study of psychiatric disorders. In very general terms, some authors have stated that all the psychiatric disorders are alterations in emotional activation ${ }^{27}$. Thus, the large amount of studies that use this type of paradigm in subjects with psychiatric diseases ${ }^{28}$ is hardly surprising.

Focusing on schizophrenia, many studies have demonstrated deficits in the emotional processing in recognition of faces ${ }^{29,30}$, and also some in affective prosody ${ }^{30}$. However, here there are also many methodological problems that make it difficult to compare the results and establish specificity, extension and nature of the deficits ${ }^{30}$. There have also been different functional neuroimaging studies that have gone deeper into the study of these deficits, both in face recognition ${ }^{31-35}$ as by pleasant and unpleasant visual stimu$\mathrm{li}^{36}$. We have not found any neuroimaging study that uses the auditory modality in the literature. This is particularly striking given that the perceptive disorders these subjects suffer are mostly within this sensorial modality.

This study presents a paradigm based on the audition of words for the study of the differences in brain activation when the content is emotional versus non-emotional. These differences are analyzed with functional neuroimaging (fMRI) and will be validated in healthy control subjects. Our final objective is for this functional neuroimaging model with emotional auditory stimulus to serve to deepen the knowledge of the underlying conditions in patients with schizophrenic psychosis who have auditory hallucinations.

\section{MATERIAL AND METHOD}

\section{Elaboration of $\mathrm{fMRI}$ paradigm}

\section{Selection of words having emotional content for psychosis}

So that the selection of the words with emotional content to be specific for psychosis, 82 patients with schizophrenia according to DSM-IV criteria and who had suffered auditory hallucinations according to their clinical record were chosen. All the patients were administered the Spanish version of the PSYRATS scale ${ }^{37}$ for hallucinations and the hallucination content were recorded on a tape recording machine. These recordings were transcribed and the most frequently appearing words were analyzed. Hallucinations that were based on complex sentences or had a neutral content were ruled out, selecting words that had meaning by themselves. A total of 65 words were chosen and were grouped according to content in five categories:

- Negative content with imperative tone (for example, get out!, kill him!)

- Insults (for example: good for nothing!, jerk!) 


\begin{tabular}{|c|c|c|c|c|}
\hline Table 1 & \multicolumn{4}{|c|}{$\begin{array}{l}\text { Studies that have used the auditory sensorial modality to analyze the emotional versus } \\
\text { non-emotional response in normal subjects }\end{array}$} \\
\hline \multicolumn{2}{|c|}{ Author and year (ref.) } & Technique & $\mathrm{N}$ & Paradigm \\
\hline \multicolumn{5}{|c|}{ Music } \\
\hline Baker et a & & PET & 10 & Different types of music \\
\hline Blood and & $2001^{11}$ & fRMI & 10 & Pleasant music \\
\hline Blood et a & & PET & 10 & Pleasant music/unpleasant music \\
\hline \multicolumn{5}{|c|}{ Noises } \\
\hline Frey et al. & & PET & 8 & Pleasant sounds/unpleasant sounds \\
\hline Hugdahl e & $95^{14}$ & PET & 5 & Noise/unpleasant tone \\
\hline Royer et a & & PET & 12 & Opinion of pleasant stimuli, including auditory \\
\hline \multicolumn{5}{|c|}{ Words } \\
\hline Imaizumi & $97^{16}$ & PET & 6 & Identification of the emotion spoken with \\
\hline Morris et & & PET & 6 & Vocalization of emotions \\
\hline Buchanan & $000^{18}$ & fRMI & 10 & Voices with different emotional intonation \\
\hline George et & & PET & 13 & Prosody of the words \\
\hline Schirmer & $04^{20}$ & RMf & 24 & $\begin{array}{l}\text { They heard a series of verbs in which emotional valency was established, } \\
\text { the stimulus varied one series with emotional tone and } \\
\text { other without it }\end{array}$ \\
\hline Philips et & & fRMI & 6 & Verbal expression of fear \\
\hline Royet et a & & PET & 12 & Opinion of pleasant stimuli, including auditory \\
\hline Isenberg e & & PET & 6 & Neutral and emotional words \\
\hline Maddock & $97^{23}$ & RMf & 7 & Words having emotional and neutral content \\
\hline Maddock & $03^{24}$ & RMf & 8 & $\begin{array}{l}\text { Words with emotional and non-emotional content, analyzing the valency } \\
\text { of them. They heard only text, words in sequential form }\end{array}$ \\
\hline Tracy et a & & fRMI & 15 & $\begin{array}{l}\text { Emotions, non-emotional words, text plus emotional words, text plus } \\
\text { non-emotional words }\end{array}$ \\
\hline Goel and & $2001^{26}$ & fRMI & 14 & Jokes with humor and without humor \\
\hline
\end{tabular}

- Imperative tone (for example: do it!, listen!)

- Exclamations related with emotional conditions (for example: fuck!, shit!)

- Positive content (for example: good!, wonderful!).

Attending to the frequency presented and given that the stimulus pattern for the fMRI experiment should last 20 seconds for each block, a total of 13 words were chosen and were distributed in the following way: 4 negative content imperative words (get out, kill him, hurl yourself down, you will die), 3 insults (whole, good for nothing, jerk), 2 with imperative tone (listen, do it), 2 exclamations related with emotions (fuck, shit), and 2 having positive content (wonderful, good).

\section{Selection of words with neutral content and pairing} with those of emotional content

The data published by S. Algarabel in which the indexes of psycholinguistic interest of 1,917 Spanish words are explained was used ${ }^{38}$. This author analyzes different types of indexes of these 1,917 words. These indexes are grouped into two large categories: objective indexes: they refer to the number of letters, number of syllables, written frequency, number of meanings in the Royal Academy dictionary and those called subjective indexes: imaginary, meaningfulness, number of attributes, concreteness, categorizability, familiarity, pleasantness. The first indexes are obtained from the mentioned sources, the subjective ones were obtained from a Valencian and Alicantinian sample of a total of 2,000 subjects who had to evaluate the words on a 1 to 7 scale. The item that interests us most here is that called pleasantness. The subjects should respond to what degree this word aroused feelings of complacence or unpleasant feelings, ranging from 1 , very unpleasant, to 7 , very pleasant.

The neutral words chosen had to have a syntactic complexity (number of syllables) similar to that of the emotional words, a similar use frequency and neutral or slightly positive score on the pleasantness scale between 3 and 4 . We also added that they should never be words that could refer to the subject if they were not common names of objects and simple concepts. The 13 words chosen were: table, 
liter, case, lamp, wheel, terrace, shoe, mountain, datum, park, meter, novel, drawing. The mean pleasantness index of the neutral words was 3.8. The total number of syllables was the same as in the emotions 33. Joining words whose association had a meaning was avoided.

To make the recording, we used a specialized center, hiring an actor to pronounce the words. The neutral words in neutral tone and the emotional ones with an emotional tone, but with the same intensity of voice ( $65 \mathrm{bs}$ ).

During the fMRI experiment, four blocks with 20 second long stimuli mixed with four more blocks with 20 seconds of rest were presented to the subject. The order of both acquisitions (emotional and neutral) was random to avoid introducing biases (adaptation, tiredness, saturation surprise) due to the order of the auditory stimulus without and with emotional content. The subjects were warned before the test that they would hear these types of words, simply asking them to pay attention to them.

\section{Functional magnetic resonance imaging technique}

\section{Design}

The neuroimaging test consisted in the acquisition of fMRI images by Blood Oxygenation Level-Dependent (BOLD) contrast, using the previously mentioned paradigm, and structured brain images having high spatial resolution to superimpose the activation maps on them.

The subjects were subjected to the block stimulation paradigm, exchanging rest states with auditory stimulation states. Figure 1 shows the distribution in time of the stimulation blocks.

This scheme of blocks was repeated in two sessions for each subject. The first acquisition was the auditory stimulation paradigm with emotional content (sequence groups of 13 words with high emotional content), the second acquisition being without emotional content (groups of 13 words with very low emotional content). Each session consisted in 80 acquisitions of tridimensional data (3D) of the total brain volume, assigning 10 consecutive acquisitions to each activation state (rest and auditory stimulation). finally, a structural volumetric sequence of the anatomical content of the brain was obtained in rest state.

\section{Acquisition of MRI images}

Data acquisition of magnetic resonance superconductor 1.5 teslas (Philips Intera 1.5, Holland) was performed by a clinical team. The patients were given headphones connected by air tubes with an audio CD reproducer. Using these headphones, the patients were isolated from the noise inherent to the fMRI experiment while they received the auditory sti-
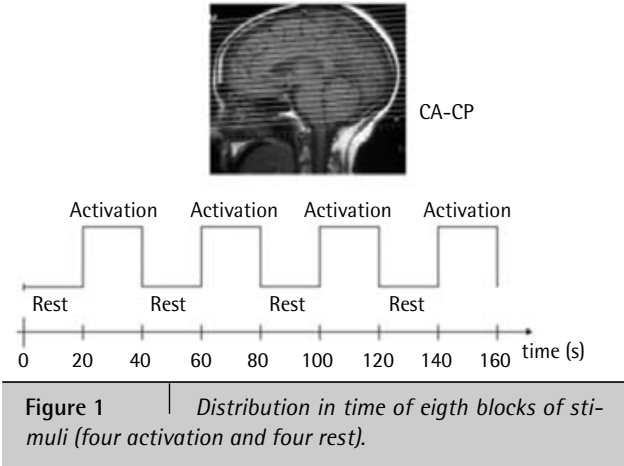

mulus. The auditory stimulus test that they were going to hear was previously described to all the subjects. The studies were acquired with the standard quadrature head coil.

For the acquisition of the functional images, the following sequence was used: $\mathrm{T}^{*}$ weighted $\mathrm{EPI}$ dynamic sequence of fMRI (multicut 2D sequence; TR: 2,000 ms; TE: $50 \mathrm{~ms} ; 5 \mathrm{~mm}$ cut thickness without separation between cuts, acquisition matrix: $96 \times 128$; field of vision (FOV): $220 \mathrm{~mm}$; excitation angle: $65^{\circ}$ ). Voxel size was $3.27 \times 1.72 \mathrm{~mm}$. Sequence was acquired with spectral suppression of fat to minimize artifacts by chemical displacement. Each one of these dynamics had 24 contiguous cuts with a parallel orientation to the anterior commissure-posterior commissure line, with a coverage of all the intracranial CNS.

To study the functional activation, a total of 80 dynamics were acquired ( 2 seconds long each one) with a global duration of the sequence of 160 seconds. Every 10 ones of these dynamics formed a block. During the experiment, four blocks in rest state and four during activation were alternately studied.

The anatomical structural image necessary for topographic localization of the activation areas was obtained with the T1 weighted gradient echo sequence (volumetric 3D acquisition; TR: $7 \mathrm{~ms}$; TE: $1.88 \mathrm{~ms}$; cut thickness: $1.2 \mathrm{~mm}$ without separation between cuts; acquisition matrix of $256 \times 256$; FOV: 220 $\mathrm{mm}$ ). With this sequence, all the intracranial nervous system is acquired with 96 cuts. The voxel size was $0.86 \times 0.86 \mathrm{~mm}$. Duration time of this sequence was 280 seconds.

\section{Data analysis}

Based on the images obtained from the structural MRI and $\mathrm{fMRI}$, an initial preprocessing was performed to improve the images in order to adequately perform the statistical analysis pixel to pixel.

The fMRI and structural images were coregistered in such a way that the anatomical areas coincided in both image mo- 
dalities. In the fMRI images, a realignment was also done with correction of subvoxel movement to eliminate the effects associated with the involuntary movement of the head during the fMRI study. Realignment of the dynamics was performed according to a reference volume. After, the images were transformed to a standard space by minimization of the quadratic error that represents the difference between the template image (MNI350, Montreal Neurological Institute) and study image. This transformation was done beginning with the structural image of each subject. The intensity of the normalized images was softened based on a tridimensional Gaussian nucleus to optimize the signal to noise ratio ${ }^{39}$, the data approaching a convenient normalized distribution for the statistical tests that were subsequently performed.

The statistical analysis consisted in the study of a single subject and the intersubject comparison (extraction of data and differences on the activation in the two paradigm groups). The analysis of the parametric maps was done with t-tests studies of a sample based on 10 healthy subjects (analysis of groups of subjects, by an Random Effects Analyses, voxel by voxel, to obtain the characteristics of common activation, according to the MLG through the SPM2 (Statistical Parametric Mapping, Wellcome Department of Imaging Neuroscience) ${ }^{40}$.

The results were filtered with the False Discovery Rate technique with a corrected $p<0.1$ and minimum threshold of a group of five voxels (groups less than five voxels were eliminated).

The activation areas were defined by Automatic Area Labeling ${ }^{41}$. Using the Wfu_pickatlas software ${ }^{42}$ mask images were obtained of each one of the regions. These were filtered for the parametric images of the test for stimuli with emotional content and the test for stimuli without emotional content, finally counting the number of active voxels.

\section{RESULTS}

Activation maps for each non-emotional/emotional paradigm were obtained from the images of the group of 10 control subjects (figs. 2 and 3). It can be observed in figure 2 how the activation is mainly located in the superior temporal gyrus when stimuli with words without emotional content are used. When the auditory stimulus is done using words with high emotional content, areas of activation were obtained in similar regions, but with a greater extension than in the case of the non-emotional paradigm, mainly in the middle and superior temporal gyrus. The meaning for the active areas in these regions reached very significant values (corrected $p<0.001$ ).

When auditory stimuli were used with emotional content, a statistically significant supplementary activation was seen in the right post-central area and in the right supramarginal gyrus that did not occur with stimuli without emotional content.

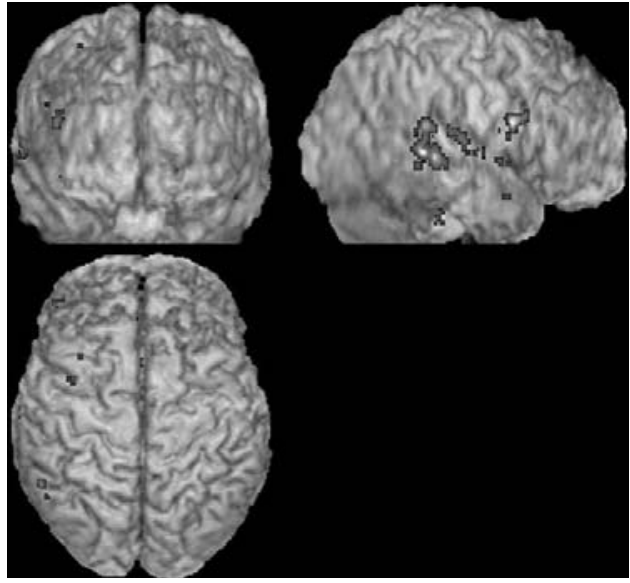

Figure $2 \quad$ Auditory activation in 10 controls with stimulation of words without emotional content.

\section{DISCUSSION}

This present article aims to present a new paradigm of emotional versus non-emotional auditory stimulation for the study of emotional reactivity in psychotic patients, showing preliminary data of $\mathrm{fMRI}$ in normal subjects. As we mentioned in the introduction, the studies reviewed on the subject have very different methodologies and thus the results are

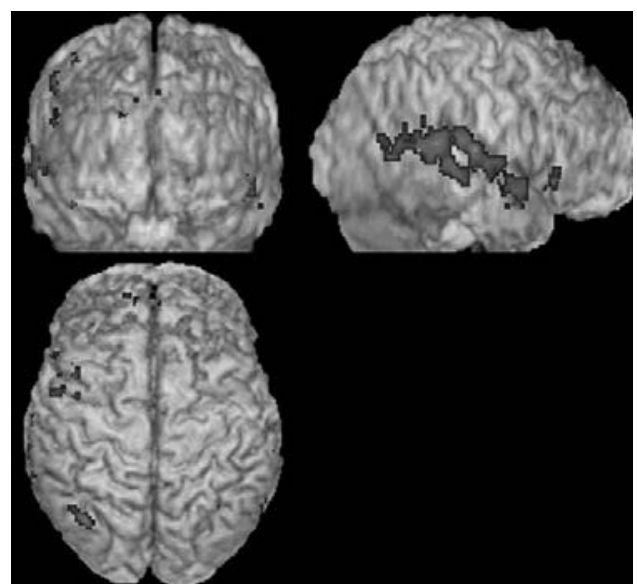

Figure 3 Auditory activation in 10 controls with stimulatin of words with emotional content. 
not comparable. The paradigm that we present herein has important differences in relationship with those previously used by other authors. The selection method of the neutral words (non-emotional) was similar to that performed by other authors, especially to that used by Maddock et al. ${ }^{24}$, although the subjects were not asked to evaluate the neutrality of these words, but rather they were selected from the data of the general population. However, the emotional words were selected based on the conversation of psychotic patients with auditory hallucinations and not from the general population nor from the previous signaling of the valency of pleasantness given by the study subjects. This determined that the words chosen had very specific characteristics. Thus, compared with other studies, the emotional words of our paradigm have the following differential characteristics: $a$ ) they have a syntactic meaning by themselves; $b$ ) all refer to the person; c) they express, without possibility of ambivalency, a strong affective content that is reinforced by a clear emotional tone (prosody), and $d$ ) as in most of the studies, there is no differentiation between whether the content of the words is pleasant or unpleasant; it is aimed to analyze the emotional filter that may be done by the psychotic patient and this may be altered both in positive and negative words.

We believe that this paradigm that is especially designed to analyze the emotional response of the psychotic patients may maximize the possible differences in emotional processing of language.

The initial results in normal subjects show a clear differentiation in cerebral activation between emotional and non-emotional words. The large increase in the activation of the temporal areas, an increase that is more marked in the right hemisphere, calls our attention in the first place. It must also be stressed that the areas of the right hemisphere as the supramarginal gyrus and the post-central area are only activated with emotional stimuli. Although these results are only preliminary and require greater analysis, they are coherent with the results of other authors $18,23,25$ and indicate that the paradigm described may be useful for the discrimination of the circuits involved in the emotional response to auditory stimuli.

If, as Maddock ${ }^{24}$ states, memory may play a very important role in the activation of these circuits, it can be expected that when psychotic subjects with auditory hallucinations listen to words that have a relationship with their hallucinations, this differentiation would be more greater. We hope to verify the certainty or non-certainty of this hypothesis in future studies.

\section{ACKNOWLEDGEMENTS}

This study was performed thanks to the structured interdisciplinary collaboration with the support of the Association for the Development and Investigation in Magnetic Resonance. This study has been financed by the Temática IM3
Network (Medical, Molecular and Multimodal Image) and by research grants from the Jansen-Cilag and Sanofi-Sinthelabo laboratories.

\section{REFERENCES}

1. Darwin C. La expresión de las emociones en los animales y en el hombre. Madrid: Alianza Editorial, 1998.

2. Ekman $P$ y Davidson RJ. The nature of Emotion. Fundamental Questions. Oxford: Oxford University Press, 1994.

3. Le Doux J. The emotional Brain. New York: Weindenfeld and Nicolson; 1998.

4. Santa Marta $C$, Desco M. Resonancia magnética funcional. En: Arango C, Crespo Facorro B, Bernardo Arroyo M, editores. Neuroimagen en psiquiatría. Madrid: Ars Medica, 2003.

5. Murphy FC, Nimmo-Smith I, Lawrence AD. Functional neuroanatomy of emotions: a meta-analysis. Cogn Affect Behav Neurosci 2003;3:207-33.

6. Breiter HC, Etcoff NL, Whalen PJ, Kennedy WA, Rauch SL, Buckner $\mathrm{RL}$, et al. Response and habituation of the human amygdala during visual processing of facial expression. Neuron 1996;17:875-87.

7. Morris JS, Frith CD, Perrett I, Rowland D, Young AW, Calder AJ, et al. A differential neural response in the human amygdala to fearful and happy facial expressions. Nature 1996;383:812-5.

8. Phillips ML, Medford N, Young AW, Williams L, Williams SC, Bullmore ET, et al. Time courses of left and right amygdalar responses to fearful facial expressions. Hum Brain Mapp 2001; 12:193-202.

9. Whalen PJ, Shin LM, Mclnerney SC, Fischer $\mathrm{H}$, Wright $\mathrm{Cl}$, Rauch SL. A functional MRI study of human amygdala responses to facial expressions of fear versus anger. Emotion 2001;1:70-83.

10. Baker SC, Frith CD, Dolan RJ. The interaction between mood and cognitive function studied with PET. Psychol Med 1997;27: 565-78.

11. Blood AJ, Zatorre RJ. Intensely pleasurable responses to music correlate with activity in brain regions implicated in reward and emotion. Proc Nat Acad Sci USA 2001;98:11818-23.

12. Blood AJ, Zatorre RJ, Bermudez P, Evans A. C. Emotional responses to pleasant and unpleasant music correlate with activity in paralimbic brain regions. Nat Neurosci 1999;2:382-7.

13. Frey $S$, Kostopoulos $P$, Petrides $M$. Orbitofrontal involvement in the processing of unpleasant auditory information. Eur J Neurosci 2000;12:3709-12.

14. Hugdahl K, Berardi A, Thompson WL, Kosslyn SM, Macy R, Baker DP, et al. Brain mechanisms in human classical conditioning: a PET blood flow study. Neuroreport 1995;6:1723-8.

15. Royet JP, Zald D, Versace R, Costes N, Lavenne, F, Koenig O, et al. Emotional responses to pleasant and unpleasant olfactory, visual, and auditory stimuli: a positron emission tomography study. J Neurosci 2000;20:7752-9.

16. Imaizumi S, Mori K, Kiritani S, Kawashima R, Sugiura M, Fukuda, H, et al. Vocal identification of speaker and emotion activates different brain regions. Neuroreport 1997;8:2809-12.

17. Morris JS, Buchel C, Dolan RJ. Parallel neural responses in amygdala subregions and sensory cortex during implicit fear conditioning. Neuroimage 2001;13:1044-52.

18. Buchanan TW, Lutz K, Mirzazade S, Specht K, Shah NJ, Zilles K, et al. Recognition of emotional prosody and verbal components 
of spoken language: an fMRI study. Brain Res Cogn Brain Res 2000;9:227-38.

19. George MS, Parekh PI, Rosinsky N, Ketter TA, Kimbrell TA, Heilman KM, et al. Understanding emotional prosody activates right hemisphere regions. Arch Neurol 1996;53:665-70.

20. Schirmer A, Zysset S, Kotz SA, Yves von Cramon, D. Gender. Differences in the activation of inferior frontal cortex during emotional speech perception. Neuroimage 2004 21:1114-23.

21. Phillips ML, Young AW, Scott SK, Calder AJ, Andrew C, Giampietro $V$, et al. Neural responses to facial and vocal expressions of fear and disgust. Proc R Soc Lond B Biol Sci 1998;265:1809-17.

22. Isenberg $N$, Silbersweig $D$, Engelien $A$, Emmerich $S$, Malavade $K$, Beattie $B$, et al. Linguistic threat activates the human amygdala. Proc Natl Acad Sci USA 1999;96:10456-9.

23. Maddock RJ, Buonocore MH. Activation of left posterior cingulate gyrus by the auditory presentation of threat-related words: an fMRI study. Psychiatry Res 1997;75:1-14.

24. Maddock RJ, Garrett AS, Buonocore MH. Posterior cingulate cortex activation by emotional words: fMRI evidence from a valence decision task. Hum Brain Mapp 2003;18:30-41.

25. Tracy J, Flanders A, Madi S, Natale P, Delvecchio N, Pyrros A, et al. The brain's response to incidental intruded words during focal text processing. Neuroimage 2003;18:117-26.

26. Goel V Dolan RJ. The functional anatomy of humor: segregating cognitive and affective components. Nat Neurosci 2001;4:237-8.

27. Panksepp J. Affective neuroscience. Oxford: Oxford University Press, 1998.

28. Phillips ML. Understanding the neurobiology of emotion perception: implications for psychiatry. Br J Psychiatry 2003;182:190-2.

29. Mandal MK, Pandey R, Prasad AB. Facial expressions of emotions and schizophrenia: a review. Schizophr Bull 1998;24:399-412.

30. Edwards J, Jackson HJ, Pattison PE. Emotion recognition via facial expression and affective prosody in schizophrenia: a methodological review. Clin Psychol Rev 2002;22:789-832.

31. Schneider F, Weiss U, Kessler C, Salloum JB, Posse, S, Grodd W, et al. Differential amygdala activation in schizophrenia during sadness. Schizophr Res 1998;34:133-42.
32. Phillips ML, Williams L, Senior C, Bullmore ET, Brammer MJ, Andrew $C$, et al. A differential neural response to threatening and non-threatening negative facial expressions in paranoid and non-paranoid schizophrenics. Psychiatry Res 1999;92:11-31.

33. Kosaka $\mathrm{H}$, Omori M, Murata $T$, Lidaka T, Yamada $H$, Okada T, et al. Differential amygdala response during facial recognition in patients with schizophrenia: an fMRI study. Schizophr Res 2002; 57(1):87-95.

34. Gur RE, McGrath C, Chan RM, Schroeder L, Turner T, Turetsky BI, et al. An fMRI study of facial emotion processing in patients with schizophrenia. Am J Psychiatry 2002;159:1992-9.

35. Kohler CG, Turner T, Bilker WB, Brensinger CM, Siegel SJ, Kanes J, et al. Facial emotion recognition in schizophrenia: intensity effects and error pattern. Am J Psychiatry 2003;160:1768-74.

36. Paradiso $\mathrm{S}$, Andreasen NC, Crespo-Facorro B, O'Leary DS, Watkins GL, Boles Ponto LL, et al. Emotions in unmedicated patients with schizophrenia during evaluation with positron emission tomography. Am J Psychiatry 2003;160:1775-83.

37. González JC, Sanjuan J, Canete C, Echanove MJ, Leal, C. La evaluación de las alucinaciones auditivas: la escala PSYRATS. Actas Esp Psiquiatr 2003;31:10-7.

38. Algarabel S. Indices de interés psicolinguistico de 1.917 palabras castellanas. Cognitiva 1996;1:43-88.

39. Turner R, Howseman A, Rees GE, Josephs O, Friston KJ. Functional magnetic resonance imaging of the human brain: data acquisition and analysis. Exp Brain Res 1998;123:5-12.

40. Tzourio-Mazoyer N, Landeau B, Papathanassiou D, Crivello F, Etard O, Delcroix N, et al. Automated anatomical labelling of activations in SPM using a macroscopic anatomical parcellation of the MNI MRI single subject brain. Neuroimage 2002;15: 273-89.

41. Maldjian JA, Laurienti PJ, Burdette JB, Kraft RA. An Automated Method for Neuroanatomic and Cytoarchitectonic Atlas-based Interrogation of fMRI Data Sets. Neurolmage 2003;19:1233-9.

42. Worsley KJ, Marrett S, Neelin P, Vandal AC, Friston KJ, Evans AC A unified statistical approach for determining significant signals in images of cerebral activation Human Brain Mapping 1996; 4:58-73. 
Figures from the article are also reproduced in color in Figure $\mathbf{1 5}$ and Figure 16.

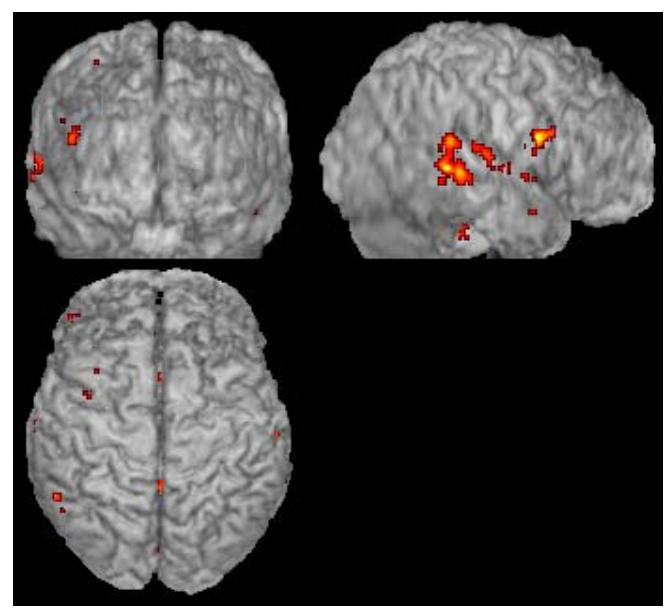

Figure 15. Auditory activation in 10 controls with stimulation of words without emotional content.

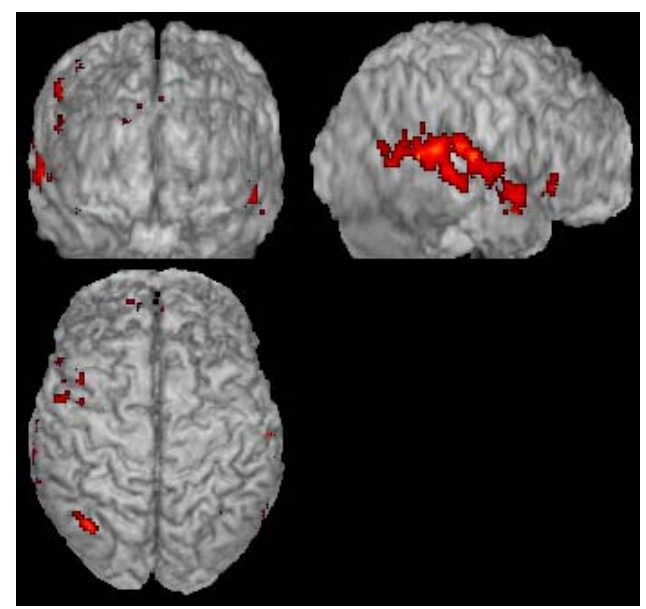

Figure 16. Auditory activation in 10 controls with stimulation of words with emotional content. 


\section{3.h. - Addendum}

As the manuscript is the main part of this chapter, some errors that have been found are transcripted here. The manuscript was not modified, as it is the one that has been already published. The addenda have been sent to the journal. Changes are presented in blue colour.

p.2-3. For example is replaced by e.g. Some words were not correctly translated:

A total of 65 words were chosen and were grouped according to content in five categories:

- Negative content with imperative tone (e.g. get out!, kill him!)

- Insults (e.g. jerk!, queer!)

- Imperative tone (e.g. do it!, listen!)

- Exclamations related with emotional conditions (for example: fuck!, shit!)

- Of positive content (e.g. good!, wonderful!).

Attending to the frequency presented and given that the stimulus pattern for the fMRI experiment should last 20 seconds for each block, a total of 13 words were chosen and were distributed in the following way: 4 negative content imperative words (get out, kill him, hurl yourself down, you will die), 3 insults (bitch, jerk, queer), 2 with imperative tone (listen, do it), 2 exclamations related with emotions (fuck, shit), and 2 having positive content (wonderful, good).

p.4 The sentence about data acquisition equipment is wrong. It should state:

Data acquisition was performed on a magnetic resonance superconductor $1.5 \mathrm{~T}$ scanner (Philips Intera 1.5, The Netherlands).

p.4 The word "cuts" appeared instead of "slices".

For the acquisition of the functional images, the following sequence was used: T2* weighted EPI dynamic sequence of fMRI (multislice 2D sequence; TR: 2,000 ms; TE: $50 \mathrm{~ms}$; $5 \mathrm{~mm}$ slice thickness without separation between slices; acquisition matrix: $96 \times 128$; field of view (FOV): $220 \mathrm{~mm}$; flip angle: $65^{\circ}$ ). Voxel size was $3.27 \times 1.72 \mathrm{~mm}$. Sequence was acquired with spectral fat suppression to minimize artefacts by chemical displacement. Each one of these dynamics had 24 contiguous slices with a parallel orientation to the anterior commissure-posterior commissure line, with a coverage of all the intracranial CNS.

In the same page: 
The anatomical structural image necessary for topographic localization of the activation areas was obtained with the T1 weighted gradient echo sequence (volumetric 3D acquisition; TR: 7 $\mathrm{ms}$; TE: $1.88 \mathrm{~ms}$; slice thickness: $1.2 \mathrm{~mm}$ without separation between slices; acquisition matrix of $256 \times 256$; FOV: $220 \mathrm{~mm}$ ). With this sequence, all the intracranial nervous system is acquired with 96 slices. The voxel size was $0.86 \times 0.86 \mathrm{~mm}$. Duration time of this sequence was 280 seconds.

p.5. Kernel was wrongly stated as nucleus. Furthermore, MLG was written instead of GLM:

The intensity of the normalized images was smoothed based on a tridimensional Gaussian kernel to optimize the signal to noise ratio $^{39}$, approximating the data to a convenient normalized distribution for the statistical tests that were subsequently performed.

The statistical analysis consisted in a single-subject analysis and an intersubject comparison (extraction of data and differences on the activation in the two paradigma groups). The analysis of the parametric maps was done with $t$-tests studies of a sample based on 10 healthy subjects (analysis of groups of subjects, by an Random Effects Analyses, voxel by voxel, to obtain the characteristics of common activation, according to the GLM through the SPM2 (Statistical Parametric Mapping, Wellcome Department of Imaging Neuroscience) $)^{40}$.

p.36 References 40, 41 and 42 in the manuscript should have the following numbering:

Maldjian et al, 2003 (40)

Worsley et al, 1996 (41)

Tzourio-Mazoyer et al, 2002 (42). 


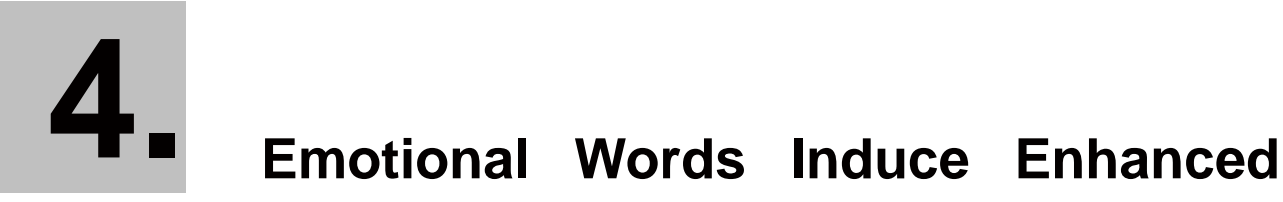 Brain Activity in Schizophrenic Patients with Auditory Hallucinations}

"Affection is responsible for nine-tenths of whatever solid and durable happiness there is in our lives" - C. S. Lewis (1898 - 1963).

The fMRI paradigm for neutral and emotional word processing (Sanjuán et al, 2005), applied to schizophrenic patients that have auditory hallucinations is presented in the following manuscript. The positive symptom (the definition of positive and negative symptoms has been presented in chapter 1) of auditory (verbal) hallucinations was selected to create a very homogeneous sample of patients with chronic auditory hallucinations. Figure 3 in the manuscript shows the large extent and amplitude in the activation that was obtained by means of the paradigm in the schizophrenic patients. The structure of this chapter is composed of an introduction to the auditory paradigm and its expected implications in the research applied to schizophrenia, and the published manuscript at last.

\section{4.a. - Auditory paradigm}

The auditory emotional speech processing paradigm was slightly outlined in Chapter 3. The development of the paradigm is described in the present chapter. The chosen paradigm was developed in the framework of the Schizophrenia Research Project (see Chapter 1) in 2003 and it was tested in volunteers and it was optimized. The final paradigm is presented in the manuscript included in this chapter.

The main aim of the Project was to detect the areas of the brain that were most influenced by auditory semantic emotional processing and to detect differences in the activation of those areas between schizophrenic and healthy 
control subjects. Words were presented auditorily. Many paradigms have studied visually presented words instead.

As the stimulation was presented auditorily and the administered stimuli were words, the brain areas that can be of interest in the study are presented below. These areas are related to audition and to language processing. Firstly the main audition area is presented and then the language interpretation area is introduced.

The Primary Auditory Cortex is located in the posteromedial part of the Heschl's gyrus (Liegeois-Chauvel et al, 1991). The Heschl's gyrus is an omegashaped small gyrus (see Figure 17, a) that is located in the Superior Temporal Gyrus (STG).
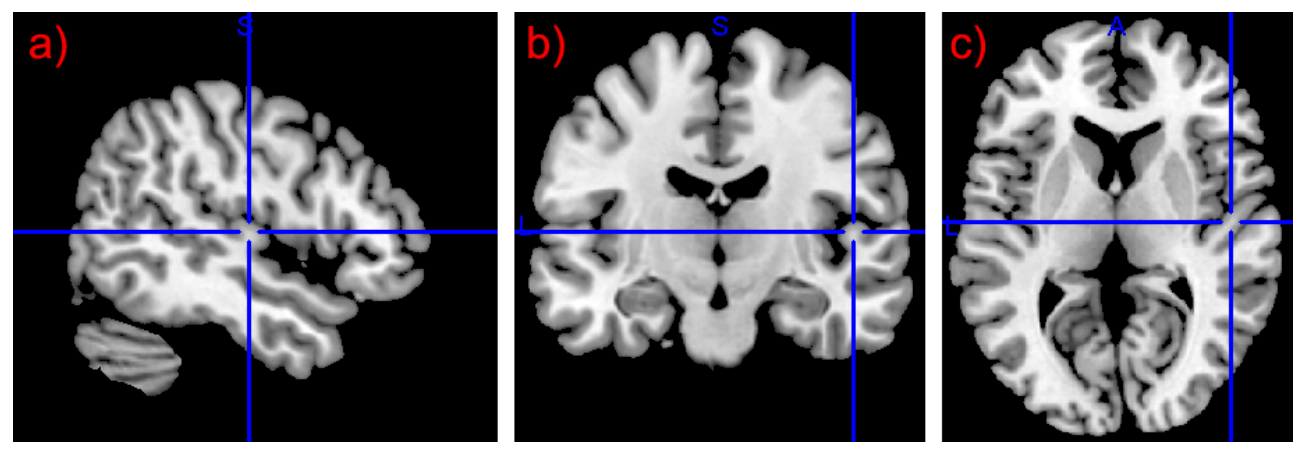

Figure 17. (Right) Heschl's gyrus identification (blue crosshairs) on an anatomical high-quality MR image, in a) Sagittal, b) Coronal and c) Axial views. The gyrus presents an omega-shape in the Sagittal view.

Primary auditory functions such as the response to pure tones and to acoustic frequency patterns map onto the medial two-thirds of the Heschl's gyrus (Morosan et al, 2001; Lütkenhöner et al, 2003). When stimuli have semantic content, then they are further processed by the Wernicke Area (Geschwind, 1979), specifically in the Planum Temporale. The network of activation then differentiates depending on the content of the stimuli.

It has been demonstrated that the kind of auditory stimulation that is applied to a subject modulates the laterality of activation in the temporal lobe. 
Human brain has a predisposition to process speech sounds in the left auditory cortex while music sounds tend to be processed in the right one (the left / right dominance depending on the function is known as functional lateralization; difference in shape between two homologous areas is known as structural lateralization). Different illnesses such as schizophrenia or specific language impairment have a different-from-normal lateralization in the temporal lobe, found both in structural and functional studies. Differences in hemispheric lateralization of function in the temporal lobe in professional musicians have also been found. These differences in lateralization indicate that both auditory processing impairments and auditory processing training generate a change in language function lateralization (for a review, see Tervaniemi and Hugdahl, 2003). The study of the activation of the brain areas that respond to auditory speech processing in healthy and schizophrenic subjects could therefore generate information about the different functionality between the healthy and the schizophrenic brains.

Audition function has been deeply explored in schizophrenia (for a review, see Shenton et al, 2001). The structure of the STG in schizophrenic subjects has also been investigated, and correlations have been found between thought disorder scores and grey matter reduction in the left posterior STG (e.g. Menon et al, 1994; Shenton et al, 1992). STG has recently been found to be altered in childhood-onset schizophrenia, demonstrating an impairment of language in non medicated subjects (Taylor et al, 2004). Gray matter deficits in STG in schizophrenic subjects have recently been found in schizophrenic patients with auditory hallucinations (Garcia-Marti et al, 2007). Furthermore, correlations between severity of auditory hallucinations and gray matter reductions in left inferior frontal gyrus and also in right postcentral gyrus were observed in that study. 


\section{4.b. - Emotion and schizophrenia}

It has been proposed that medical treatments do not remove symptoms of schizophrenia, such as auditory hallucinations, but they rather regulate the salience given to emotional stimuli by psychotic patients (Kapur, 2003). This regulation of salience is achieved through medicines that regulate dopamine, a neurotransmitter related to several brain functions that plays a role in Parkinson disease and psychotic illnesses, amongst others (Schultz, 2007). The emotional response has been studied in schizophrenia with neuroimaging methods. A recent study (Plaze et al, 2006) found that severity of hallucinations negatively correlated with activation in the left temporal superior region when listening to neutral sentences. The finding supports the hypothesis that auditory hallucinations compete with normal external speech for processing sites within the temporal cortex in schizophrenia.

Fakra and colleagues (2008) and Williams and colleagues (2007) explored emotional response in schizophrenia by presenting facial emotional stimuli. The first research group detected a failure in the activation of regions of the limbic system implicated in the automatic processing of emotions in schizophrenic patients when the subjects had to match emotional faces (Fakra et al, 2008). The study by Williams and colleagues found that schizophrenia patients displayed abnormally increased phasic arousal (measured by means of skin conductance response), with concomitant reductions in fMRI-measured activation in emotionspecific regions and Medial Prefrontal cortex (MPFC) (Williams et al, 2007). Lee and colleagues (2006) also found different activation in schizophrenic subjects in the MPFC in a social cognition paradigm. Most of the fMRI studies about schizophrenia performed to date involved experimental designs traditionally used in neuropsychology (Niznikiewicz et al, 2003). The majority of studies that used sensory stimulation did so through the recognition of facial emotions (Murphy et al., 2003). However, relatively few of them have used the auditory modality. Emotional response with regard to the neural basis of auditory hallucinations needs further attention (Allen et al, 2008). 


\section{4.c. - Emotional words. An emotional semantic auditory paradigm}

A paradigm that replicated the experience of emotions in schizophrenic subjects was developed. With this aim, emotional and neutral stimuli were selected that would be administered auditorily. Firstly, the psychiatry group obtained the most usual words a cohort of more than two hundred schizophrenic subjects usually heard. Then they selected the words in terms of emotional salience (Algarabel, 1996) and word complexity (only two and three syllable words were accepted). Thirteen high emotional words were finally chosen from the words schizophrenic patients heard, and thirteen neutral words were matched to the emotional ones, from the list generated by Algarabel. The words were recorded by a Spanish-dubbing professional. The two blocks of words were randomized separately in four different blocks of twenty seconds each. The distribution of words in one of the two sequences can be observed in Figure 18, where intensity of sound shows the distribution of words along time.

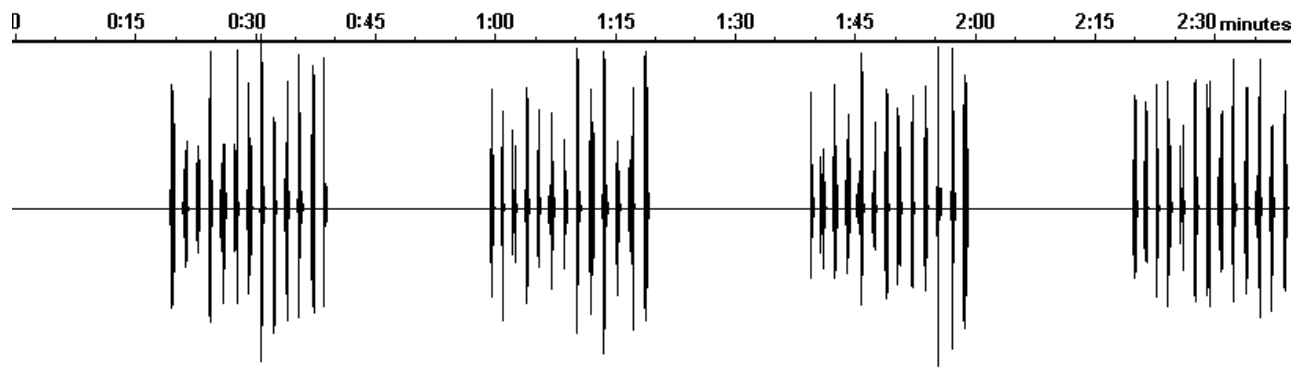

Figure 18. Temporal distribution and sound intensity of the words presented in one sequence (emotional sequence is displayed).

\section{4.d. - Data analysis}

In addition to the data analysis presented in the manuscript, alternative methods were tested by the $\mathrm{PhD}$ candidate. Independent Component Analysis method (ICA) has been tested on the schizophrenic population fMRI data with help of S. Coello (Coello et al, 2004). 
The discriminating power between schizophrenic subjects and healthy control subjects' fMRI maps was also explored (Navarro et al, 2006). With this aim, the SPM for each subject was used as an input to an analysis of separability between groups. The SPM were separated by means of Principal Component Analysis (PCA) and Fisher's Linear Discriminant (FLD). The dataset comprised 15 right handed male schizophrenic patients with chronic auditory hallucinations (with DSM-IV schizophrenia) and 15 paired healthy control subjects.

A Region of Interest (ROI) was drawn selecting only the temporal lobes in the spatially normalized data. An ROI was drawn by means of MRIcro, an opensource useful tool by Christian Rorden (Rorden, Brett, 2000). The ROI was applied as a mask to the SPMs. Each masked SPM had 149.532 voxels. If each voxel was assumed to be an independent variable, the dimensionality became too high for timely comparisons with FLD; therefore a dimensionality reduction approach was used. PCA was used on a matrix $\boldsymbol{X}$ where rows were the number of subjects ( $n$ ) and columns were the activation map voxels $(m)$. A matrix $\boldsymbol{D}$ was constructed whose rows were subjects and whose columns were projections onto a selected number of principal components (PC). Selection was done by discarding the components with the smallest eigenvalues.

An alternative robust PCA variation algorithm for the case where $\mathrm{n}$ is much less than $\mathrm{m}$ was used, as in (Ford et al, 2001). FLD analysis was performed in order to classify the subjects.

A leave-one-out (LOO) approach was carried out to test the FLD classifier.

Finally, for each LOO trial, the distance between the test subject projection and the training projections (separately for patients and controls, finding the minimal distance in each case) was used to predict whether a subject was a control or a patient.

With the PCA and FLD method we found that discrimination between schizophrenic patients and control subjects by means of fMRI maps (70\% of 
subjects correctly classified) was possible by means of selecting the appropiate voxels, reducing the data dimensionality by PCA and using the FLD classifier.

Optimal number of principal components to be taken to the FLD was explored experimentally. With this aim, FLD was tested once with each possible number of principal components (components were selected including the first one, then the two first components... until the ten first components). With one principal component (the most informative one), percentage of correctly classified subjects was around $35 \%$, less than a random classifier would perform. When adding more components, the percentage of correctly classified subjects varied until the eight most informative components were selected, giving a $70 \%$ of subjects correctly classified, as stated in the above paragraph. When more components were introduced the percentage of subjects correctly classified decreased.

Areas with highest discriminant power are shown in Figure 19. These areas were selected as follows: First, voxels that have been most important in the separation of components in the data, performed by PCA, were selected. When selecting a number of components (eight in this case), the voxels that contain the most part of the information that constitute the components can be extracted. This is obtained by multiplying the component mixture by the data. Then a threshold was applied, with a 0.008 arbitrary threshold. Finally, clusters with fifty voxels or more were thresholded into the map shown in Figure 19. 


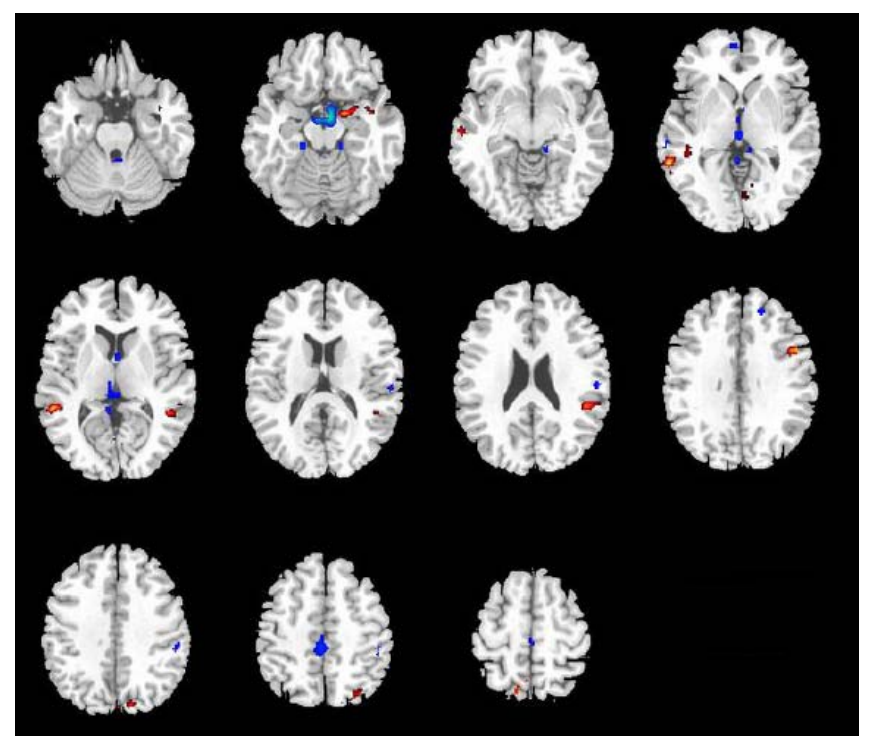

Figure 19. Areas that discriminate best between chronic hallucinatory patients and healthy subjects, by means of the PCA-FLD approach, are shown in red and blue in axial slices. Red clusters show areas higher than 0.008 while blue clusters show areas lower than -0.008 .

\section{4.e. - Process automation}

Finally, in addition to alternative analysis methods, as the number of subjects included in the study was by far higher than the number in the study presented in the previous chapter, automation was introduced in the processing of the fMRI data. An automation tool for preprocessing and statistical analysis was created and published on the Internet by the PhD candidate: http://www.fil.ion.ucl.ac.uk/spm/ext/\#spm2batchJJ).

fMRI data acquisition-quality assessment techniques were introduced (i.e. movement threshold assessment, detection of artefacts), by means of Matlab scripts. Even though maximum movement to be allowed in a subject during an fMRI session depends on the task to be evaluated, it is advisable to select a threshold, as movement correction algorithms reduce the movement but they can not take all movement out of the images. Movement that is correlated to the stimulation paradigm will introduce changes in the signal intensity that may be 
detected as brain activation. Head motion introduces image shifts and field inhomogeneity variations which cause local changes in geometric distortions. Those distortions appear near junctions between air and tissue, especially prominent in the frontal sinuses and in the ear canals. Rigid body movement correction will not be able to remove such artefacts, as there exist movement-byinhomogeneity interactions (methods have been developed for correcting for such artefacts, mainly by "unwarping" the EPI images: see Andersson et al, 2001). Furthermore, movement is a source of false positives in the fMRI statistical analyses (Desmond, Atlas, 2000). Matlab code was generated by the PhD candidate that tested for difference in movement between slices higher than half of the voxel size. Mean and peak movement through each session was also assessed, by means of the reports that movement correction algorithms generate. fMRI data that surpassed the maximum allowed movement were discarded from the study. Automated labelling was also introduced for the classification of activation areas in the brain by means of the AAL software (Tzourio-Mazoyer et al, 2002): Anatomical variability between subjects is high; therefore an automated labelling based on the $\mathrm{MNI}$ template helped in the identification of the activation areas. 


\section{4.f. - PhD candidate contributions}

The tasks carried out by the $\mathrm{PhD}$ candidate in the elaboration of the manuscript were:

- Collaboration in the fMRI data acquisition.

- Pre-processing of the fMRI data. Quality assessment for every subject's fMRI data.

- First level (subject-wise) and second-level (multisubject) statistical analyses following the General Linear Model in a voxel-by-voxel basis.

- Generation of figures two and three in the article and Table 1 in the text.

- Collaboration in the editing of the manuscript, mainly in the Methods and Results sections.

\section{Emotional words induce enhanced brain activity in schizophrenic patients with auditory hallucinations}

Julio Sanjuán, Juan J. Lull, Eduardo J. Aguilar, Luis Martí-Bonmatí, David MoratalPérez, José C. González, Montserrat Robles, Matcheri S. Keshavan.

Psychiatry Res 2007. 154(1):21-9 


\title{
Emotional words induce enhanced brain activity in schizophrenic patients with auditory hallucinations
}

\author{
Julio Sanjuan $^{\mathrm{a}, *}$, Juan J. Lull ${ }^{\mathrm{b}}$, Eduardo J. Aguilar ${ }^{\mathrm{c}}$, Luis Martí-Bonmatí ${ }^{\mathrm{d}}$, \\ David Moratal $^{\mathrm{b}}$, José C. Gonzalez ${ }^{\mathrm{c}}$, Montserrat Robles ${ }^{\mathrm{b}}$, Matcheri S. Keshavan ${ }^{\mathrm{e}}$

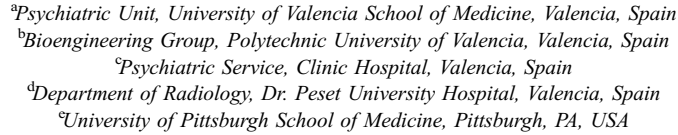

Received 24 March 2005; received in revised form 25 October 2005; accepted 21 April 2006

\begin{abstract}
Neuroimaging studies of emotional response in schizophrenia have mainly used visual (faces) paradigms and shown globally reduced brain activity. None of these studies have used an auditory paradigm. Our principal aim is to evaluate the emotional response of patients with schizophrenia to neutral and emotional words. An auditory emotional paradigm based on the most frequent words heard by psychotic patients with auditory hallucinations was designed. This paradigm was applied to evaluate cerebral activation with functional magnetic resonance imaging (fMRI) in 11 patients with schizophrenia with persistent hallucinations and 10 healthy subjects. We found a clear enhanced activity of the frontal lobe, temporal cortex, insula, cingulate, and amygdala (mainly right side) in patients when hearing emotional words in comparison with controls. Our findings are consistent with other studies suggesting a relevant role for emotional response in the pathogenesis and treatment of auditory hallucinations.

(C) 2006 Elsevier Ireland Ltd. All rights reserved.
\end{abstract}

Keywords: Schizophrenia; Functional MRI; Salience; Emotional paradigm; Auditory hallucinations

\section{Introduction}

Auditory hallucinations $(\mathrm{AH})$ are nuclear symptoms in psychoses. Recently, the whole range of functional imaging techniques has been used to evaluate the functional anatomy of the hallucinatory experience. Several areas have been implicated including the auditory cortex in functional magnetic resonance imaging (fMRI)

* Corresponding author. Unidad de Psiquiatría, Facultad de Medicina, Universidad de Valencia, 46010 Valencia, Spain. Tel.: +34 963983379; fax: +34 963864767.

E-mail address: julio.sanjuan@uv.es (J. Sanjuan). studies (Woodruff et al., 1995), Broca's area in a single proton emission computed tomography (SPECT) study (McGuire et al., 1993), and subcortical structures in a positron emission tomography (PET) study (Silbersweig et al., 1995). Neurobehavioral covariables such as the response to outer auditory speech (Woodruff et al., 1997), the monitoring of the inner verbal image (McGuire et al., 1996) and the discrimination of inner-outer space (Hunter et al., 2003) have also been evaluated through functional imaging studies.

Although the underlying biological mechanisms remain unclear, $\mathrm{AH}$ are thought to be related with the activation of cerebral areas involved in normal

0925-4927/\$ - see front matter (C) 2006 Elsevier Ireland Ltd. All rights reserved. doi:10.1016/j.pscychresns.2006.04.011 
processing of auditory stimuli. However, some studies also suggest a broader activation of cortical and subcortical areas (Woodruff, 2004). Several neurocognitive models have been proposed to explain auditory verbal hallucinations in schizophrenia: abnormal auditory imagery, dysfunction in self-monitoring, or abnormalities in storing and retrieving of memories (Seal et al., 2004).

Emotional response has recently been implicated in the pathogenesis of AH (Kapur, 2003). Moreover, the emotional factor is crucial in cognitive therapy of patients with AH (Freeman and Garety, 2003).

A wide range of neuroimaging paradigms have been used to study emotional response in normal subjects. They have included different sensory modalities and cognitive tasks. Along with classical works in this field (Ekman and Davidson, 1994), most of the studies have used the visual sensory modality through the recognition of facial emotions (Murphy et al., 2003). However, relatively few of them (Maddock and Buonocore, 1997; Isenberg et al., 1999) have used the auditory modality, despite the importance of language in human emotions.

Some studies have shown deficits in patients with schizophrenia in regard to emotional processing in facerecognition tasks, and also with affective prosody (Mandal et al., 1998; Edwards et al., 2002). However, several methodological problems make it difficult to compare results and to identify the specificity, extension and nature of these deficits (Edwards et al., 2002). Some fMRI studies have used paradigms based on face recognition (Schneider et al., 1998; Phillips et al., 1999; Kosaka et al., 2002; Gur et al., 2002); emotionally aversive and non-aversive pictures, including faces (Taylor et al., 2002); and pleasurable or non-pleasurable olfactory (Crespo-Facorro et al., 2001) or visual stimuli (Paradiso et al., 2003). All these studies have generally shown a widespread decrease of brain activation in patients with schizophrenia compared with controls.

As far as we know, no emotion-induction auditory paradigm has been used in a neuroimaging study in patients with schizophrenia, despite the fact that $\mathrm{AH}$ are present in $70-80 \%$ of such patients (Slade and Bentall, 1988). In this fMRI study, we compared brain activation of patients with $\mathrm{AH}$ and healthy controls using a new auditory emotional paradigm especially designed for psychotic patients with AH. The principal aim is to evaluate the response to neutral and emotional words. In global terms, although patients usually experience fear and perplexity towards $\mathrm{AH}$, some comment on them as a pleasurable experience (Sanjuan et al., 2004); further, the familiarity and memory of the stimulus could be important in emotional response. For these reasons, we used a paradigm derived from voices and included some positive-pleasant words.

We predicted an increased activation of limbic brain regions in both the chronic hallucinators and the controls, when exposed to emotional words compared with when they were presented with neutral words. We also expected a different pattern of activation between patients and controls, reflecting underlying mechanisms that could play a role in the emotional response to $\mathrm{AH}$ and, therefore, in their pathogenesis itself.

\section{Materials and methods}

\subsection{Subjects}

A group of 22 male psychotic chronic hallucinators were selected out of a sample of 106 patients with $\mathrm{AH}$. All subjects gave written informed consent to participate in the research. The study was approved by the local ethics committee. The characteristics of this sample are described elsewhere (Sanjuan et al., 2004). All of the patients met the following selection criteria for persistent hallucinations:

(a) Voices were not modified in any way by treatment over the course of a year.

(b) Voices were present at least once a day in the last year.

(c) At least two antipsychotic drugs had been tried, at doses equivalent to $600 \mathrm{mg}$ /day of chlorpromazine, in the last year.

For this study, and in order to get a homogeneous group, only patients who heard voices during data acquisition at the end of the fMRI were included $(n=14)$. Three patients were excluded because of gross movement during fMRI data acquisition. Healthy controls were matched by age, gender (all males), laterality (all right-handed) and educational level to the patients. Subjects with a psychiatric history or presence of perceptual abnormalities were not considered as controls. No individual in either group suffered from hearing loss.

The final sample included 11 patients with DSM-IV schizophrenia (American Psychiatric Association, 1994) and 10 healthy controls from a similar ethnic group and educational level. Patients' educational levels were as follows: Illiterate $=1(9.1 \%)$, Primary $=6$ $(54.5 \%)$, Secondary $=3(27.3 \%)$, University $=1$ (9.1\%). Only one patient was married $(9.1 \%)$, another one was divorced $(9.1 \%)$, and nine were unmarried $(81.8 \%)$. Their ages ranged from 21 to 51 years (mean 38.3, S.D. 7.2), 
while ages at which patients began to hear $\mathrm{AH}$ ranged from 15 to 43 years (mean 23.0, S.D. 10.0). The mean duration of illness was 14.7 years (S.D. $=8.1$ ). All patients were under antipsychotic treatment time of evaluation: 6 $(55.5 \%)$ under second-generation antipsychotics, and 5 (44.5\%) under combined treatment (first- and secondgeneration antipsychotics).

All patients were clinically assessed with the Global Assessment Scale (GAS) (Endicott et al., 1976), 24item Brief Psychiatric Rating Scale (BPRS) (Overall and Gorham, 1962), Positive and Negative Syndrome Scale (PANSS) (Kay et al., 1987), and Psychotic Symptom Rating Scale (PSYRATS) for AH (Haddock et al., 1999) over the last $24 \mathrm{~h}$. The PSYRATS scale was administered just before data acquisition. The BPRS mean score was 55.2 (range 41-67, S.D. 7.4), the PANSS mean score was 70.8 (range 53-94, S.D. 9.9), the PSYRATS mean score was 28.6 (range 20-34, S.D. 4.4), and the GAS mean score was 38.5 (range 20-45, S.D. 8.5).

At the end of the trial, every patient was asked to score the frequency of voices during MRI, the resemblance of the voices to his own voices, and the level of anxiety. Control subjects were also asked the last question.

\subsection{Selection of emotional and neutral words}

An emotional response paradigm was designed to replicate those emotions related to hallucinatory experiences. Eighty-two patients with schizophrenia meeting DSM-IV criteria with $\mathrm{AH}$ were selected in order to choose words of emotional content specific to their psychoses. All patients were administered the PSYRATS and their discourses about the content of $\mathrm{AH}$ were recorded on tape. The recordings underwent transcription. Qualitative data were analyzed using the methodology proposed by Miles and Huberman (1994). Hallucinations based on complex phrases or with neutral content were ruled out. A total of 65 words were chosen based on their frequency, including only those possessing meaning by themselves. They were classified according to the qualitative analysis of their content in five categories: of negative content and imperative tone, insults, of imperative tone, and exclamations related to emotional states and of positive content.

Given that the stimuli pattern for the fMRI experiment lasts $20 \mathrm{~s}$ for each block, a total number of 13 words were selected according to their frequency in the recording, and then grouped as follows: four imperative words of negative content, three insults, two words with imperative tone, two exclamations related to emotions, and two words of positive content. For the selection of neutral words, we used data published by Algarabel (1996) in which the rate of psychological interest of 1917 Spanish words was described. Subjective rates were obtained from a group of 2000 subjects (from Valencia and Alicante, Spain) who evaluated words on a scale from 1 to 7 . The most relevant item for this study was "pleasantness". Subjects had to answer to which degree the word triggered pleasant or unpleasant feelings, on a scale in which $1=$ very unpleasant and $7=$ very pleasant. The pleasantness average rate of neutral words was 3.8. The pleasantness average rate of emotional selected words was 1.4 for words of negative content, 1.2 for insults, 1.5 for words with imperative tone, 2.1 for exclamations related to emotions, and 5.8 for words of positive content. Emotional and neutral words' valences were significantly different as shown by a paired $t$-test $(t=-3.09, d f=12$, $P=0.009)$. Finally, the total number of syllables $(n=33)$ coincided with the number of syllables in the emotional words $(n=33)$.

For the recording procedure, a professional actor from a specialized center was hired to pronounce the words. He pronounced neutral words using a neutral tone and emotional words using an emotional tone but maintaining voice intensity constant $(65 \mathrm{~dB})$.

\subsection{Image acquisition}

The fMR images were obtained by means of BOLD (Blood Oxygenation Level Dependent) (Ogawa et al., 1992) contrast, applying the stimulation paradigm described before. Subjects were binaurally stimulated in two different sessions. Fig. 1 represents the distribution of the blocks for both sessions in time. The activation blocks in the first session consisted of 13 Spanish words containing high emotional content. The second session had activation blocks containing 13 words having neutral or low emotional content.

Four blocks of stimuli, $20 \mathrm{~s}$ each, interleaved with another four blocks of rest of $20 \mathrm{~s}$ each, were presented to patients and controls (Fig. 1). The acquisition order (emotional and neutral) was randomized to avoid biases (habituation, fatigue, saturation and surprise). Subjects were informed before the test about the two types of words they were going to listen to, and were asked to focus their attention on them. A MR 1.5-Tesla. (Philips Medical Systems, Holland) was used for data acquisition. Patients had earphones adjusted to their heads. These earphones were connected by a pair of air tubes to an external audio $\mathrm{CD}$ player.

Images were performed applying a dynamic Echo Planar Imaging T2* weighted sequence $(\mathrm{TR}=2000 \mathrm{~ms}$, $\mathrm{TE}=50 \mathrm{~ms}, 5 \mathrm{~mm}$ slice thickness with no inter-slice gap, acquisition matrix $=96 \times 128$, field of view $=220 \mathrm{~mm}$ 

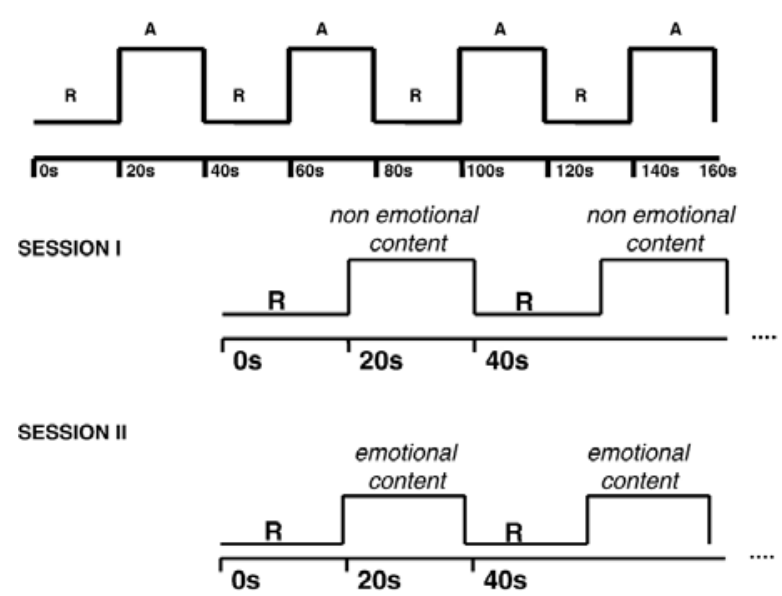

Fig. 1. Stimulation paradigm.

and flip angle $=65^{\circ}$ ). The voxel size was $3.27 \mathrm{~mm} \times$ $1.72 \mathrm{~mm}$. The sequence was obtained with spectral suppression. Each dynamic acquisition consisted of 24 contiguous slices parallel to the anterior-posterior commissural plane, covering the whole brain.

Additional high-resolution volumetric images were acquired to have an anatomical template of the whole brain. The anatomical high-resolution MR images, necessary for the topographical localization of the activation areas, were obtained with a Gradient Echo T1- weighted sequence (3D volumetric acquisition, $\mathrm{TR}=7 \mathrm{~ms}$, $\mathrm{TE}=1.88 \mathrm{~ms}$, slice thickness $1.25 \mathrm{~mm}$ without inter-slice gap, $256 \times 256$ acquisition matrix, $\mathrm{FOV}=220 \mathrm{~mm}$ ). A total of 96 slices with a voxel size of $0.86 \mathrm{~mm} \times 0.86 \mathrm{~mm}$ were obtained.

\subsection{Data analysis}

Processing was carried out with the SPM2 (Statistical Parametric Mapping Functional Imaging Laboratory, London) (Friston et al., 1995). Automatic labeling was applied to the following group maps: patients that were presented high-emotional content, patients presented neutral content control subjects presented high-emotional content, and control subjects presented neutral content.

MR images were initially processed to allow voxelbased statistical analyses. Functional images were realigned with subvoxel movement correction. Images were co-registered for every subject so that structural and functional images were situated in exactly the same virtual space. Images were then transformed into standard space, minimizing the least square error that represents the difference between the template image (MNI150, Montreal Neurological Institute) and the subject's image.

Image intensity was smoothed by means of a Gaussian three-dimensional 6-mm kernel, approaching the data to a normal distribution necessary for later statistical tests. Statistical analysis was performed first on each individual subject and also through comparison between subjects (extraction of information and differences in activation between subject groups). The voxel-based parametric maps analysis was performed with one sample $t$-tests from the final sample of 10 control subjects and 11 patients (Random Effects Analyses applied to each group comparison), extracting common features, following the General Linear Model ( $d f=9$ for controls, $d f=10$ for patients; $t$-test values, see Table 1).

The False Discovery Rate technique was applied, using the whole brain to address the problem of multiple comparisons, retaining voxels surviving with a value of $P<0.05$ and a minimum cluster of five voxels.

Areas of activation were delimited with the atlas proposed by Schmahmann et al. (1999). This atlas is included in the software Automatic Area Labeling (Tzourio-Mazoyer et al., 2002), which extracts a table 
Table 1

Areas of activation with emotional words, BOLD signal, in hallucinating patients with schizophrenia and controls*

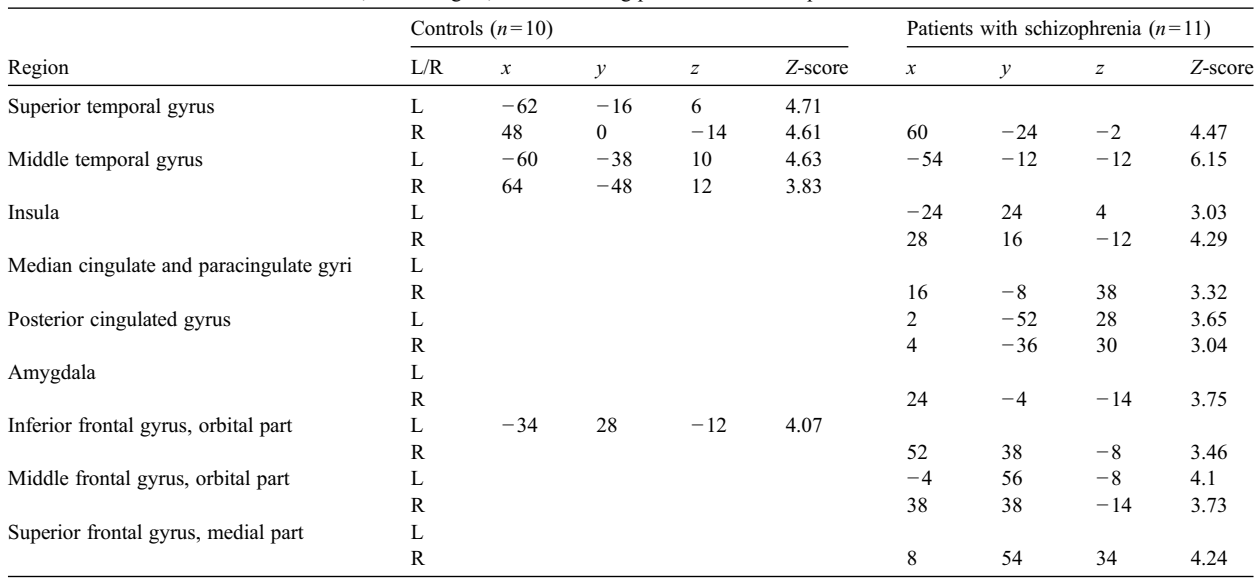

${ }^{*}$ Coordinates are MNI.

with all local maxima of activation and the areas they correspond to in the labeled atlas. If the voxel estimated as active was not in any of the areas labeled, it was assigned to the nearest labeled area. Voxels farther than $4 \mathrm{~mm}$ of a labeled area were discarded.
Maps of significant differences in BOLD signal in all schizophrenic patients and controls between emotional content stimuli and baseline, and also between nonemotional content stimuli and baseline, were then calculated (Figs. 2 and 3). Corrected values of $P<0.05$

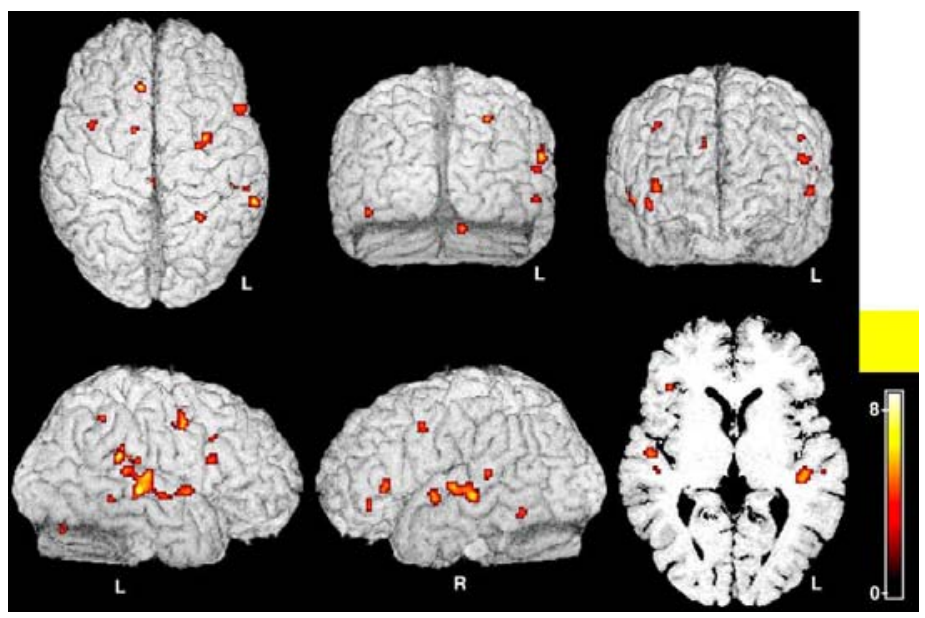

Fig. 2. Activation maps in chronic schizophrenic patients with AH, under non-emotional content stimuli. Areas with functional response are mainly the middle right cingulum, left superior and middle temporal gyri and left precentral area (after $P$ value correction, no suprathreshold voxels were found. Therefore, for illustrational purposes, a $P<0.001$ uncorrected was applied, with minimum voxel size per cluster 5). 


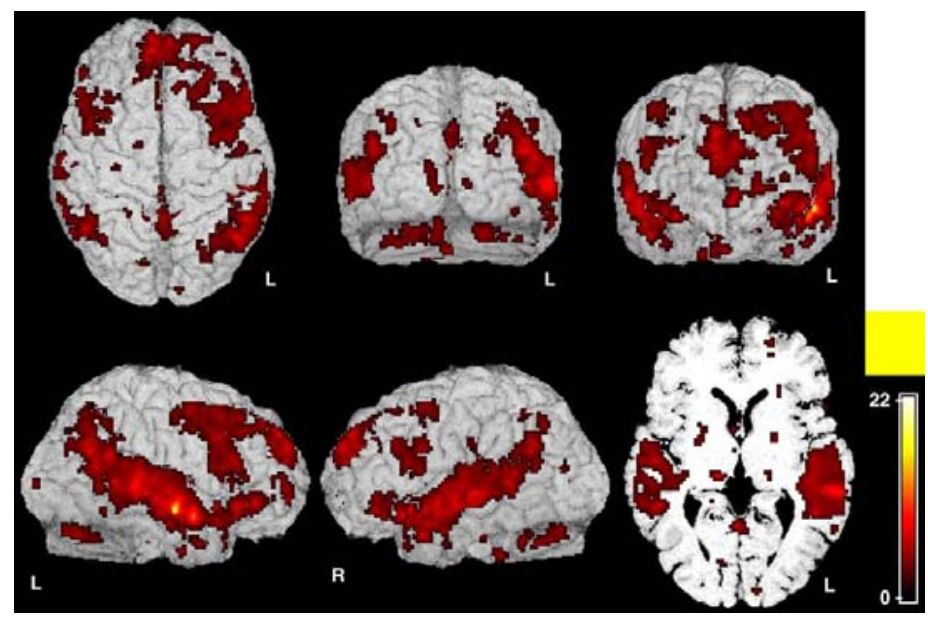

Fig. 3. Activation maps in schizophrenia patients with chronic auditory hallucinations under emotional auditory content. Note the larger activation clusters in the left middle and right superior temporal lobes, right insula and thalamus, and middle and superior frontal lobes $($ Table 1$)(P<0.05$, FDRcorrected, minimum voxel size per cluster 5).

were applied for controls (emotional and non-emotional paradigms) and patients (emotional paradigm).

\section{Results}

In regard to the questions asked immediately after MR data acquisition, results were as follows:

\section{a) Patients}

- Frequency of voices during the MR experiment: none $=0$, some $=4(36.4 \%)$, often $=4(36.4 \%)$, almost continuously $=2(18.2 \%)$, all the time $=1$ $(9.1 \%)$.

- Resemblance of voices to their own voices: none $=0$, a little resemblance $=5(45.5 \%)$, some resemblance $=2(18.2 \%)$, a lot of resemblance $=4$ $(36.4 \%)$, identical $=0$.

- Level of anxiety: none $=1(9.1 \%)$, very little $=2$ $(18.2 \%)$, slight anxiety $=4(36.4 \%)$, moderate anxiety $=3(27.3 \%)$, severe anxiety $=1(9.1 \%)$.

b) Controls

- Level of anxiety: none $=4(40 \%)$, very little $=3$ $(30 \%)$, slight anxiety $=2(20 \%)$, moderate anxiety $=1(10 \%)$, severe anxiety $=0$.

Neutral words against rest baseline patients' group map did not show any activation with the threshold applied $(P<0.05)$ when non-emotional words were presented. After application of a less stringent $P$ value $(P<0.001)$ in order to see activation trends, main areas involved were left middle and left superior temporal gyrus, middle cingulate gyrus and left inferior frontal gyrus at the orbital part (Fig. 2). When patients were presented high emotional content stimuli, activation survived the $P<0.05$ corrected threshold and was greater both in extent and in estimated activation power. Middle left temporal gyrus was the main involved area. Other areas included right superior temporal gyrus, left and right insula, right median cingulate, right and left posterior cingulate, right amygdala, right and left middle frontal cortex at the orbital part, right inferior frontal cortex at the orbital area, and superior medial cortex (Table 1, Fig. 3).

Activation maps in control subjects did not show striking differences between emotional and non-emotional paradigms. In both conditions, the main areas involved were right and left superior temporal gyri, and right and left temporal gyri, while left inferior frontal gyrus at the orbital part of left insula was activated only with non-emotional paradigm (Table 1).

\section{Discussion}

The main result of our study is the clearly enhanced activity of the orbitofrontal cortex, temporal cortex, insula, cingulate, and amygdala (mainly right side) in 
patients when hearing emotional words in comparison with controls. Our finding that the left middle temporal gyrus was the main involved area when patients were presented high emotional content stimuli is concordant with the hypothesis of a reversal of function in schizophrenia in respect of prosody, which is usually considered to rely on the right hemisphere in healthy subjects (Woodruff, 2004). However, activation of the right superior temporal gyrus is probably more related to hallucinations and is concordant with previous reports (Woodruff et al., 1995).

The activations of the insula and orbitofrontal cortex were not surprising since these areas are key regions for the emotional response (Woodruff, 2004) and have also been related to hallucinations (Woodruff et al., 1995; Shergill et al., 2004). Contrary to our predictions, right middle cingulate, and right and left posterior cingulate were activated, but not the anterior cingulate. The activation of the posterior cingulate by threat-related words has been hypothesized to engage episodic memory processes in healthy volunteers (Maddock and Buonocore, 1997). On the other hand, right amygdala's activation has frequently been reported associated with $\mathrm{AH}$ and could also be explained as an evocation of the adverse emotional response to these phenomena (Woodruff, 2004).

Recently, several articles have tried to clarify the neural circuits responsible for the processing of personal salience (significance of stimuli to the individual). Bechara et al. (2003) proposed a neurobiological model of endogenous salience by differentiating primary (intrinsic) and secondary inducer processing, although both can overlap. Phan et al. (2004) have supported and extended their model in healthy subjects. Our results are partially concordant with their conclusions. According to these authors, the insula would be responsible for converging both primary and secondary inducer processing. This would be congruent with the relevance of the insula in our schizophrenic patients, since voices heard by subjects probably behaved as primary and secondary inducers in patients and just as primary inducers in controls. It may be that activations in the posterior cingulate and the amygdala reflect the retrieval of emotional verbal memory during an experience similar to the patients' hallucinations. In any case, the selfrelatedness of stimuli is probably crucial in emotional processing in patients with schizophrenia. In our paradigm, all the emotional words were self-related and had a strong emotional content.

Most previous studies using emotional paradigms in patients with schizophrenia show a decrease of activation in comparison with controls (Schneider et al., 1998; Phillips et al., 1999; Crespo-Facorro et al., 2001; Gur et al., 2002; Kosaka et al., 2002; Paradiso et al., 2003). Our results based on an auditory emotional paradigm showed the opposite. There could be two main reasons for these differences: differences in the stimuli and in the samples.

Differences in the stimuli: Most previous studies have used a visual modality with paradigms of emotional faces (Phillips et al., 1999; Gur et al., 2002; Kosaka et al., 2002) or pleasant and unpleasant pictures (Taylor et al., 2002; Paradiso et al., 2003). Visual stimuli are more likely to be influenced by attention bias, which is frequent in patients with schizophrenia (Salgado-Pineda et al., 2004). Moreover, auditory emotional stimuli could be more appropriate to activate emotional response in schizophrenia because of the symptom profile. This could be especially true since in our study the emotional words are taken from the most frequent emotional voices.

Taylor et al. (2002) found that patients had greater activation of the medial prefrontal cortex when viewing pictures from the International Affective Picture System. Reduced or enhanced activity could be related to the emotional intensity of the stimuli. In other words, there could be a trend to have polar responses: little activation with normal social cognition tasks (Russell et al., 2000) and too much activation if the intensity or selfrelatedness of the emotional stimuli is strong enough.

Differences in the samples: All the patients in our study suffered chronic and persistent AH. All had a high score on positive symptoms. Our patients' BPRS scores $($ mean $=56)$ are much higher than in other neuroimaging emotional studies such as those of Phillips et al. (1999) $($ mean $=23)$ and Taylor et al. (2002) (mean=39).

Previous data in other studies are concordant with Bleuler's cardinal feature of schizophrenia, blunted affect (Lane, 2003), showing a decrease in brain activation or anhedonia (Crespo-Facorro et al., 2001). However, this decrease in brain activation cannot explain positive symptoms. The functional enhanced activation of our patients could be directly related to the emotional response to positive symptoms. Our findings suggest the importance of emotional response in the development and treatment of psychotic symptoms (Birchwood et al., 2000; Garety et al., 2001; Freeman and Garety, 2003). They may explain the observation of worsening psychoses in high expressed emotion families in schizophrenia (Falloon, 1988).

Our data are of interest in light of Kapur's recent proposal on the possible mechanism of action of antipsychotics (Kapur, 2003). Kapur postulated that in psychosis a dysregulated dopamine transmission leads 
to an aberrant assignment of salience to external objects or internal representation. Antipsychotics are efficacious in psychosis because they "dampen salience" of the subjective experience of delusion and hallucinations. It is noticeable that all the patients of our sample have persistent $\mathrm{AH}$ in spite of having been treated with antipsychotics for a long period. It could be possible that patients stabilized with antipsychotics would not have had this enhanced activation effect.

It was of interest that when neutral stimuli were applied, no clear activation was found. A reason for this could be that patients were being continuously activated by their own voices. If we reduce the threshold of significance, an activation of the left temporal lobe appears. This attenuation of response to neutral auditory stimuli had already been described in the study of David et al. (1996).

A limitation of our study is that these results are generalizable to schizophrenic patients with persistent auditory hallucinations and not to schizophrenia or psychosis as a whole group. It will be important to replicate this study in less severely ill patients and those who have never experienced hallucinations. Schizophrenic patients with a clinical history of hallucinations also need to be investigated using these paradigms before and after stabilization of hallucinations.

In conclusion, our findings suggest an enhanced activation of the limbic and frontal brain areas in persistently hallucinatory patients using a specific selfrelated auditory emotional paradigm. These findings may help us in our understanding of the dysfunction of the emotional response in schizophrenia.

\section{References}

Algarabel, S., 1996. Indices de interés psicolinguístico de 1917 palabras castellanas. Cognitiva 1, 43-88.

American Psychiatric Association, 1994. Diagnostic and Statistical Manual of Mental Disorders, 4th edition. APA, Washington, DC.

Bechara, A., Damasio, H., Damasio, A.R., 2003. Role of the amygdala in decision-making. Annals of the New York Academy of Sciences 985, 356-369.

Birchwood, M., Meaden, A., Trower, P., Gilbert, P., Plaistow, J., 2000. The power and omnipotence of voices: subordination and entrapment by voices and significant others. Psychological Medicine 30, 337-344.

Crespo-Facorro, B., Paradiso, S., Andreasen, N.C., O'Leary, D.S., Watkins, G.L., Ponto, L.L., Hichwa, R.D., 2001. Neural mechanisms of anhedonia in schizophrenia: a PET study of response to unpleasant and pleasant odors. JAMA 286, 427-435.

David, A.S., Woodruff, P.W., Howard, R., Mellers, J.D., Brammer, M., Bullmore, E., Wright, I., Andrew, C., Williams, S.C., 1996. Auditory hallucinations inhibit exogenous activation of auditory association cortex. NeuroReport 7, 932-936.
Edwards, J., Jackson, H.J., Pattison, P.E., 2002. Emotion recognition via facial expression and affective prosody in schizophrenia: a methodological review. Clinical Psychology Review 22, 789-832.

Ekman, P., Davidson, R.J., 1994. The Nature of Emotion. Fundamental Questions. Oxford University Press, Oxford.

Endicott, J., Spitzer, R.L., Fleiss, J.L., Cohen, J., 1976. The Global Assessment Scale: a procedure for measuring overall severity of psychiatric disturbance. Archives of General Psychiatry 33, 766-771.

Falloon, I.R., 1988. Expressed emotion: current status. Psychological Medicine 18, 269-274.

Freeman, D., Garety, P.A., 2003. Connecting neurosis and psychosis: the direct influence of emotion on delusions and hallucinations. Behaviour Research and Therapy 41, 923-947.

Friston, K.J., Holmes, A.P., Worsley, K.J., Poline, J.P., Frith, C.D., Frackowiak, R.S.J., 1995. Statistical parametric maps in functional imaging: a general linear approach. Human Brain Mapping 2, 189-210.

Garety, P.A., Kuipers, E., Fowler, D., Freeman, D., Bebbington, P.E., 2001. A cognitive model of the positive symptoms of psychosis. Psychological Medicine 31, 189-195.

Gur, R.E., McGrath, C., Chan, R.M., Schroeder, L., Turner, T., Turetsky, B.I., Kohler, C., Alsop, D., Maldjian, J., Ragland, D., Gur, R.C., 2002. An fMRI study of facial emotion processing in patients with schizophrenia. American Journal of Psychiatry 159, 1992-1999.

Haddock, G., McCarron, J., Tarrier, N., Faragher, E.B., 1999. Scales to measure dimensions of hallucinations and delusions: the Psychotic Symptom Rating Scales (PSYRATS). Psychological Medicine 29, 879-888.

Hunter, M.D., Griffiths, T.D., Farrow, T.F., Zheng, Y., Wilkinson, I.D., Hedge, N., Woods, W., Spence, S.A., Woodruff, P.W., 2003. A neural basis for the perception of voices in external auditory space. Brain 126, 161-169.

Isenberg, N., Silbersweig, D., Engelien, A., Emmerich, S., Malavade, K., Beattie, B., Leon, A.C., Stern, E., 1999. Linguistic threat activates the human amygdala. Proceedings of the National Academy of Sciences of the United States of America 96, 10456-10459.

Kapur, S., 2003. Psychosis as a state of aberrant salience: a framework linking biology, phenomenology, and pharmacology in schizophrenia. American Journal of Psychiatry 160, 13-23.

Kay, S.R., Fiszbein, A., Opler, L.A., 1987. The Positive and Negative Syndrome Scale (PANSS) for schizophrenia. Schizophrenia Bulletin 13, 261-276.

Kosaka, H., Omori, M., Murata, T., Iidaka, T., Yamada, H., Okada, T., Takahashi, T., Sadato, N., Itoh, H., Yonekura, Y., Wada, Y., 2002. Differential amygdala response during facial recognition in patients with schizophrenia: an fMRI study. Schizophrenia Research 57, 87-95.

Lane, R.D., 2003. The neural substrates of affect impairment in schizophrenia. American Journal of Psychiatry 160, 1723-1725.

Maddock, R.J., Buonocore, M.H., 1997. Activation of left posterior cingulate gyrus by the auditory presentation of threat-related words: an fMRI study. Psychiatry Research: Neuroimaging 75, $1-14$.

Mandal, M.K., Pandey, R., Prasad, A.B., 1998. Facial expressions of emotions and schizophrenia: a review. Schizophrenia Bulletin 24, 399-412.

McGuire, P.K., Shah, G.M., Murray, R.M., 1993. Increased blood flow in Broca's area during auditory hallucinations in schizophrenia. Lancet 342, 703-706. 
McGuire, P., Silbersweig, D.A., Wright, I., Murray, R.M., Frackowiak, R.S., Frith, C.D., 1996. The neural correlates of inner speech and auditory verbal imagery in schizophrenia. Relationship to auditory verbal hallucinations. British Journal of Psychiatry 169, 148-159.

Miles, M.B., Huberman, A.M., 1994. Qualitative Data Analysis. Sake Publications, Thousand Oaks.

Murphy, F.C., Nimmo-Smith, I., Lawrence, A.D., 2003. Functional neuroanatomy of emotions: a meta-analysis. Cognitive, Affective and Behavioral Neuroscience 3, 207-233.

Ogawa, S., Tank, D.W., Menon, R., Ellermann, J.M., Kim, S.G., Merkle, H., Ugurbil, K., 1992. Intrinsic signal changes accompanying sensory stimulation: functional brain mapping with magnetic resonance imaging. Proceedings of the National Academy of Sciences of the United States of America 89, $5951-5955$.

Overall, J.E., Gorham, D.R., 1962. The Brief Psychiatric Rating Scale. Psychological Reports 10, 799-812.

Paradiso, S., Andreasen, N.C., Crespo-Facorro, B., O'Leary, D.S., Watkins, G.L., Boles Ponto, L.L., Hichwa, R.D., 2003. Emotions in unmedicated patients with schizophrenia during evaluation with positron emission tomography. American Journal of Psychiatry 160, 1775-1783.

Phan, K.L., Taylor, S.F., Welsh, R.C., Ho, S.H., Britton, J.C., Liberzon, I., 2004. Neural correlates of individual ratings of emotional salience: a trial-related fMRI study. NeuroImage 21, $768-780$.

Phillips, M.L., Williams, L., Senior, C., Bullmore, E.T., Brammer, M. J., Andrew, C., Williams, S.C., David, A.S., 1999. A differential neural response to threatening and non-threatening negative facial expressions in paranoid and non-paranoid schizophrenics. Psychiatry Research: Nueroimaging 92, 11-31.

Russell, T.A., Rubia, K., Bullmore, E.T., Soni, W., Suckling, J., Brammer, M.J., Simmons, A., Williams, S.C., Sharma, T., 2000. Exploring the social brain in schizophrenia: left prefrontal reduced activity during mental state attribution. American Journal of Psychiatry $157,2040-2042$.

Salgado-Pineda, P., Junque, C., Vendrell, P., Baeza, I., Bargallo, N., Falcon, C., Bernardo, M., 2004. Decreased cerebral activation during CPT performance: structural and functional deficits in schizophrenic patients. NeuroImage 21, 840-847.

Sanjuan, J., Gonzalez, J.C., Aguilar, E.J., Leal, C., van Os, J., 2004. Pleasurable auditory hallucinations. Acta Psychiatrica Scandinavica $110,273-278$.
Schmahmann, J.D., Doyon, J., McDonald, D., Holmes, C., Lavoie, K., Hurwitz, A.S., Kabani, N., Toga, A., Evans, A., Petrides, M., 1999. Three-dimensional MRI atlas of the human cerebellum in proportional stereotaxic space. NeuroImage 10, 233-260.

Schneider, F., Weiss, U., Kessler, C., Salloum, J.B., Posse, S., Grodd, W., Müller-Gartner, H.W., 1998. Differential amygdala activation in schizophrenia during sadness. Schizophrenia Research 34, $133-142$.

Seal, M.L., Aleman, A., McGuire, P., 2004. Compelling imagery, unanticipated and deceptive memory: neurocognitive models of auditory verbal hallucinations in schizophrenia. Cognitive Neuropsychiatry 9, 43-72.

Shergill, S.S., Brammer, M.J., Amaro, E., Williams, S.C., Murray, R. M., McGuire, P.K., 2004. Temporal course of auditory hallucinations. British Journal of Psychiatry 185, 516-517.

Silbersweig, D., Stern, E., Frith, C., Cahill, C., Holmes, A., Grootoonk, S., Seaward, J., McKenna, P., Chua, S.E., Schnorr, L.A., 1995. Functional neuroanatomy of hallucinations in schizophrenia. Nature 378, 176-179.

Slade, P., Bentall, R., 1988. Sensory Deception: A Scientific Analysis of Hallucination. Croom Helm, London.

Taylor, S.F., Liberzon, I., Decker, L.R., Koeppe, R.A., 2002. A functional anatomic study of emotion in schizophrenia. Schizophrenia Research 58, 159-172.

Tzourio-Mazoyer, N., Landeau, B., Papathanassiou, D., Crivello, F., Etard, O., Delcroix, N., Mazoyer, B., Joliot, M., 2002. Automated anatomical labelling of activations in spm using a macroscopic anatomical parcellation of the MNI MRI single subject brain. NeuroImage 15, 273-289.

Woodruff, P.W., 2004. Auditory hallucinations: insights and questions from neuroimaging. Cognitive Neuropsychiatry 9, 73-91.

Woodruff, P.W., Brammer, M., Mellers, J., Wright, I., Bullmore, E., Williams, S.C., 1995. Auditory hallucinations and perception of external speech. Lancet 346, 1035.

Woodruff, P.W., Wright, I.C., Bullmore, E.T., Brammer, M., Howard, R.J., Williams, S.C., Shapleske, J., Rossell, S., David, A.S., McGuire, P.K., Murray, R.M., 1997. Auditory hallucinations and the temporal cortical response to speech in schizophrenia: a functional magnetic resonance imaging study. American Journal of Psychiatry $154,1676-1682$. 


\section{4.g. - Addendum}

There are two errors that have been detected in the manuscript (pages are referred to the manuscript numbering).

p.21 The definition of SPECT was wrong. It should be:

SPECT - Single Photon Emission Computed Tomography

p.52 The word Holland appeared instead of 'The Netherlands'.

A MR 1.5 Tesla (Philips Medical Systems, The Netherlands) 


\section{4.h. - Supplementary results}

A direct comparison between control and schizophrenic groups was performed, selecting emotional processing. In the subject level, a contrast was generated that tested the subtraction between emotional condition and neutral conditions. This contrast was then submitted to a two sample t-test that compared the two study groups testing controls subjects > schizophrenic patients. Another two sample t-test compared schizophrenic patients > controls subjects. No voxels survived a 0.05 FDR multiple comparison correction in any of the two statistical maps. Therefore, a $\mathrm{p}<0.001$ uncorrected threshold was set and results are considered as exploratory.

Table 1. Two sample t-test between control subjects and schizophrenic patients, emotional processing. Control subjects > schizophrenic patients; emotional condition $>$ neutral condition; $p<0.001$ uncorrected.

\begin{tabular}{|c|c|c|c|c|c|c|}
\hline$x$ & y & $\mathbf{z}$ & Area & Hemisphere & $\mathbf{T}$ & $\mathbf{p}$ \\
\hline 22 & -18 & -4 & Thalamus & $\mathrm{R}$ & 4,31 & $8,35 e-06$ \\
\hline-34 & 50 & -2 & Frontal Middle Orbital & $\mathrm{L}$ & 4,08 & $2,25 \mathrm{e}-05$ \\
\hline-40 & -42 & 8 & Temporal Superior & $\mathrm{L}$ & 4,01 & $3,00 \mathrm{e}-05$ \\
\hline-30 & 18 & 4 & Insula & $\mathrm{L}$ & 3,98 & $3,38 e-05$ \\
\hline 46 & 4 & -28 & Temporal Middle & $\mathrm{R}$ & 3,93 & $4,16 \mathrm{e}-05$ \\
\hline 10 & -50 & 22 & Precuneus & $\mathrm{R}$ & 3,91 & $4,54 \mathrm{e}-05$ \\
\hline 22 & 20 & 32 & Frontal Superior & $\mathrm{R}$ & 3,83 & $6,39 \mathrm{e}-05$ \\
\hline 4 & -46 & -24 & Vermis_1_2 & $\mathrm{R}$ & 3,72 & $9,77 \mathrm{e}-05$ \\
\hline 10 & -46 & -38 & Cerebelum_9 & $\mathrm{R}$ & 3,69 & 0,0001 \\
\hline 44 & -32 & 16 & Temporal Superior & $\mathrm{R}$ & 3,67 & 0,0001 \\
\hline-22 & -32 & -6 & Hippocampus & $\mathrm{L}$ & 3,65 & 0,0001 \\
\hline-56 & -60 & 34 & Angular & $\mathrm{L}$ & 3,59 & 0,0002 \\
\hline 52 & -50 & 2 & Temporal Middle & $\mathrm{R}$ & 3,57 & 0,0002 \\
\hline 6 & -38 & 4 & Lingual & $\mathrm{R}$ & 3,46 & 0,0003 \\
\hline 16 & -24 & 4 & Thalamus & $\mathrm{R}$ & 3,43 & 0,0003 \\
\hline 44 & 28 & 18 & Frontal_Inf_Tri & $\mathrm{R}$ & 3,32 & 0,0005 \\
\hline 14 & -4 & -18 & Amygdala & $\mathrm{R}$ & 3,3 & 0,0005 \\
\hline 34 & 20 & -12 & Insula & $\mathrm{R}$ & 3,28 & 0,0005 \\
\hline
\end{tabular}


Table 2. Two sample t-test: Control subjects < schizophrenic patients; emotional condition $>$ neutral condition; $\mathrm{p}<0.001$ uncorrected.

\begin{tabular}{llllcll}
$\mathbf{x}$ & $\mathbf{y}$ & $\mathbf{z}$ & Area & Hemisphere & $\mathbf{T}$ & $\mathbf{p}$ \\
25 & -68 & 14 & Calcarine & $\mathrm{R}$ & 3.43 & 0.0001 \\
\hline
\end{tabular}


MR Analysis of the Coincidence between Functional and Morphological Abnormalities in Schizophrenic Patients with Chronic Auditory Hallucinations

"Coincidences are spiritual puns" - G.K. Chesterton (1874-1936).

A new method for extracting information on the areas of the brain with damage, both functional and anatomical, in the subjects with schizophrenia, has been developed. The method combines information from functional and structural brain imaging by mixing the mostly active regions nearby the ones with biggest gray matter decrease. Eight regions were selected with this method as very probably related to schizophrenia illness: left and right middle temporal and superior temporal gyri, left posterior and right anterior cingular gyri, left inferior frontal gyrus, and middle occipital gyrus.

The principal results of this work have been published in Radiology. The published manuscript is the core of this chapter. In the first part of the chapter, the methodology that has been used to determine the brain areas where there is a coincidence between gray matter reduction (in the schizophrenic group compared to the healthy group) and a difference in brain activation in response to emotional semantic auditory stimuli between the same groups. Afterwards, in the second part of the chapter, an alternative approach to the search of coincidences between VBM and fMRI is explained. The final part consists of the manuscript.

\section{5.a. - Multimodal image analysis}

In the Schizophrenia Research Project, data from different MR techniques have been acquired, explored and published. By one side, fMRI differences between schizophrenic and healthy subjects have been found (Sanjuán et al, 
2007). By the other side, differences in brain structure have been explored by means of the Voxel Based Morphometry (VBM) method (Garcia-Marti et al, 2008). In general, there is a relative lack of available multimodal image analysis methodologies (Casanova et al, 2007). Lastly, some methods have been proposed that deal with multimodal image analysis, such as Biological Parametric Mapping (Casanova et al, 2007), a tool that generally integrates different modalities into the GLM approach (as an example implementation, fMRI and VBM modalities are integrated in a GLM in the manuscript, in a study about dyslexia); pattern recognition techniques, such as the ones proposed by Fan et al (2007) in a study of brain abnormality by combining fMRI and structural MR data; and algorithms to display damaged areas observed in coinciding functional, anatomical and metabolic images (Sabbah et al, 2002).

Our approach was similar to the work by Sabbah and colleagues (2002). Even though one possible explanation for common changes in function and structure in a specific area is that one is a consequence of the other one (i.e. structural changes change function, so function is abnormal), another possible explanation is inter-related changes in function and structure in the specific area. In the presented manuscript, this last approach is the one that has been investigated. Statistical group maps generated in the study of function (by means of fMRI) and structure (by means of VBM) have been collapsed in one summary map that has been called "coincidence map". Differences in emotion semantic processing (as presented in Chapter 4) function between schizophrenic subjects and healthy control subjects were first encoded in a SPM (fMRI SPM) that showed the t values from a two-sample t test. Gray matter deficits in schizophrenic patients compared to healthy subjects were also introduced in a SPM (VBM SPM), with the higher the $t$ values the higher the decrease in gray matter in patients compared to control subjects. The VBM SPM was produced by means of the optimized VBM method (Good et al, 2001; Ashburner, Friston, 2000).

The FMRI SPM and the VBM SPM were then smoothed and a coincidence image was generated that accounted for both changes in function and in structure in schizophrenic patients compared to healthy subjects. The coincidence map was calculated by a multiplication of both SPM maps, so differences that were common 
in both modalities would clearly appear while differences that only appeared in one modality would not be present in the final map. With this process, six areas of interest were found to have decreased gray matter density and impaired functioning in schizophrenia.

\section{5.b. - Other multimodality analysis experiments}

Reduced structural density and $\mathrm{fMRI}$ aberrant activation was also studied by means of joint fMRI and VBM-based ROI methods (Lull et al, 2006). Twenty four chronic male schizophrenia patients and controls (12 patients, 12 controls), group matched for age, were explored by means of $\mathrm{FMRI}$ and anatomical MRI (acquisition sequences as described in Chapters 3 and 4). fMRI analyses (SPM2) were restricted to 11 schizophrenic patients who reported hallucinations during the fMRI scan and 10 healthy controls. Random effects analysis of individual contrast images (emotional $>$ neutral) revealed clusters of greater activation $(p<.05, F D R)$ in schizophrenic patients in temporal lobe structures including the middle temporal gyrus (MTG; 50,-48, 2), hippocampus (26, -32, 4), and fusiform gyrus (-22,-38,-12). These areas can be observed in Figure 20 , in axial slices. To investigate structural integrity of the hyper-activated area of the MTG, a spherical region of interest (ROI) was created around the corresponding to the largest-estimated activation voxel (radius $=15 \mathrm{~mm}$ ), as observable in Figure 21.

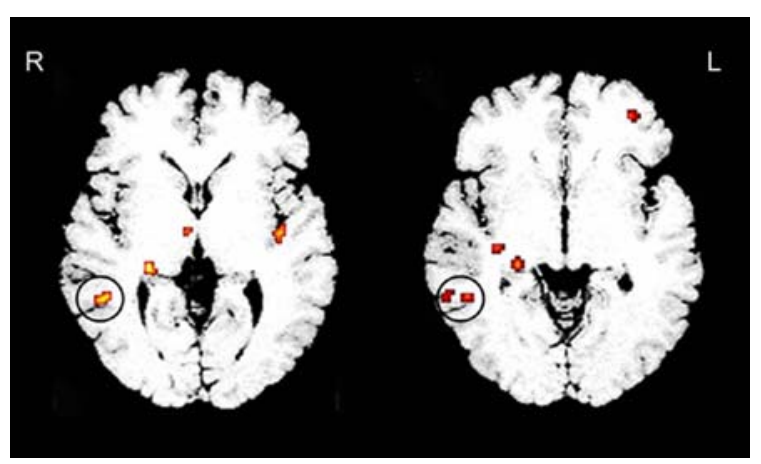

Figure 20. Middle Temporal Gyrus maximum in the fMRI SPM (contrast: estimated activation in schizophrenic subjects $>$ healthy subjects; emotional $>$ neutral). 


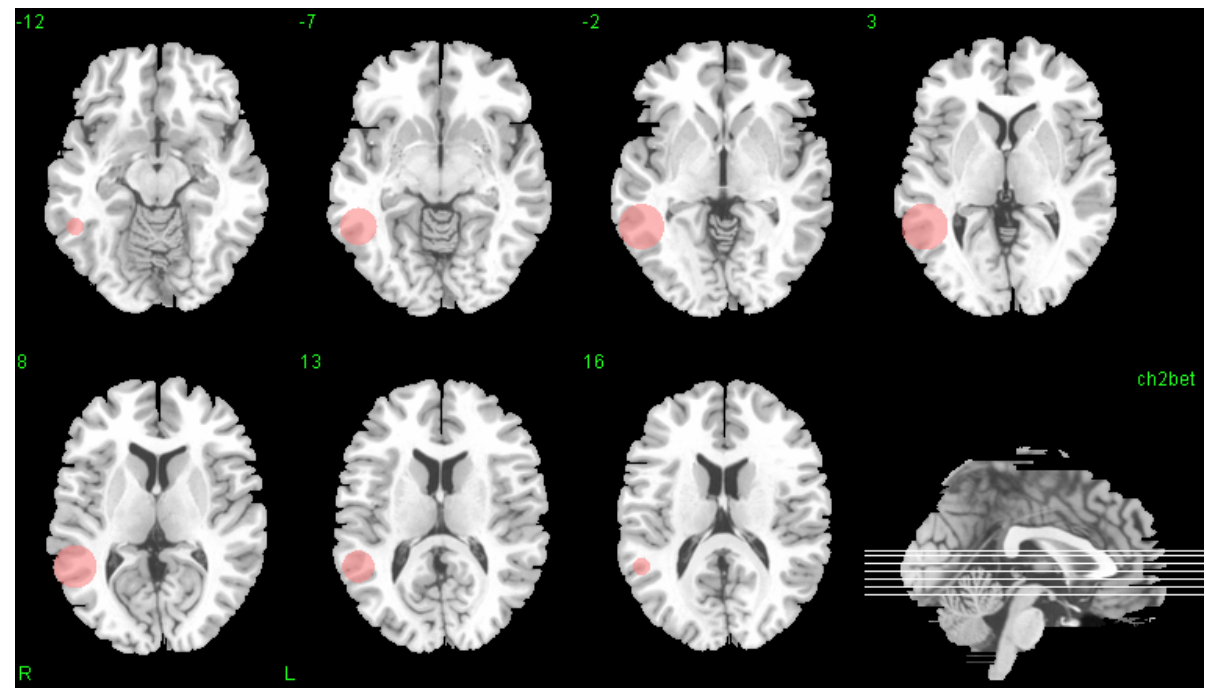

Figure 21. ROI generated around the maximum estimated activation in the MTG. $\mathrm{ROI}$ is shown in pale red on axial slices.

Each subjects' segmented gray matter image (following normalization and segmentation using VBM optimized protocol) was masked using the generated ROI, and cumulative gray matter concentration values (within the ROI) were submitted to an ANOVA revealing significant reductions in schizophrenic subjects $(F(1,34)=4.55, p<.05)$. These results suggested that $\mathrm{fMRI}$ hyper activation may be strongly associated with the presence of structural alterations.

The MTG was also detected in the results presented in the manuscript as an area with both changes in gray matter density and in activation between groups. The temporal lobe is a region that is very important in schizophrenia, with both subcortical and neocortical areas consistently reported as altered in their structure (Shenton et al, 2001). Many areas have been found to act abnormally in the schizophrenic brain, depending on the stimulation type that was applied and on the selection criteria of schizophrenic subjects. The observation of widespread activation changes is consistent with the existence of a subtle change in the synaptic organization of the brain in schizophrenia (Harrison, 2005). A thorough study of the interrelation between temporal lobe structure changes and functional activation differences is proposed as a follow-up of this study. 


\section{5.c. - PhD candidate contributions}

The tasks carried out by the $\mathrm{PhD}$ candidate in the elaboration of the manuscript, as stated in the text at p.75, were:

Collaboration in fMRI data acquisition.

Pre-processing of the fMRI data. Quality assessment for every subject's fMRI data.

- $\quad$ Collaboration in literature research.

- $\quad$ First level (subject-wise) and second-level (multisubject) statistical analyses following the General Linear Model in a voxelby-voxel basis to the fMRI data.

- $\quad$ Generation of figures two and three in the article and Table 1 in the text.

Collaboration in the editing of the manuscript.

\section{Chronic Auditory Hallucinations in Schizophrenic Patients:}

\section{MR Analysis of the Coincidence between Functional and Morphologic Abnormalities}

Luis Martí-Bonmatí, Juan J. LulI, Gracián García-Martí, Eduardo J. Aguilar, David Moratal-Pérez, Cecilio Poyatos, Montserrat Robles, Julio Sanjuán.

Radiology 2007. 244(2):549-56. 


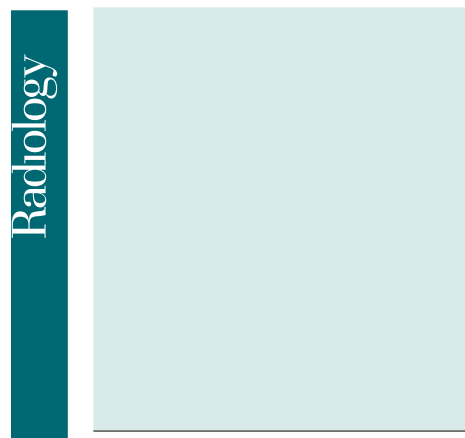

Luis Martí-Bonmatí, MD Juan José Lull, MD Gracián García-Martí, MD

Eduardo J. Aguilar, MD David Moratal-Pérez, MD Cecilio Poyatos, MD Montserrat Robles, MD Julio Sanjuán, MD
Chronic Auditory Hallucinations in Schizophrenic Patients: MR Analysis of the Coincidence between Functional and Morphologic Abnormalities $^{1}$

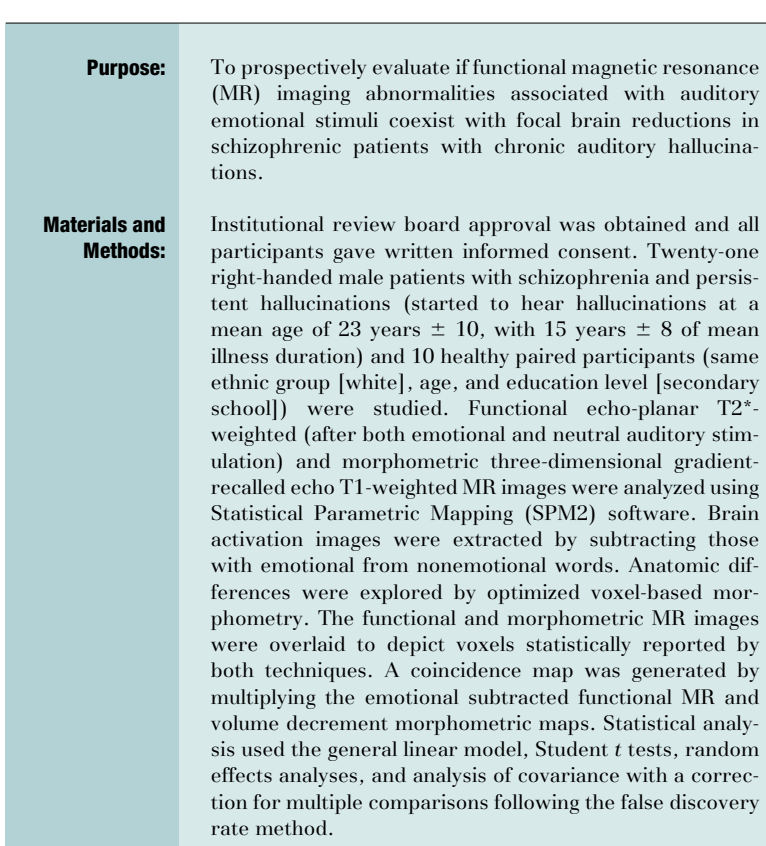

Results:

${ }^{1}$ From the Department of Radiology, Dr Peset University Hospital, Avda Gaspar Aguilar 90, 46017 Valencia, Spain (L.M., C.P.); BET Group, Polytechnic University of Valencia, Valencia, Spain (J.J.L., G.G., D.M., M.R.); and Psychiatric Unit, University of Valencia School of Medicine, Valencia, Spain (E.J.A., J.S.). Received April 26, 2006; revision requested June 23; revision received August 7

accepted September 7; final version accepted December 4. Supported by Spanish grant FIS P.I. 2005, PI 052332 and IM3 (Spanish National Network, Instituto de Salud Carlos III, G03/185). Address correspondence to L.M. (e-mail: Luis.Marti@uv.es).

- RSNA, 2007

Radiology: Volume 244: Number 2-August 2007 
chizophrenia is a heterogeneous disorder affecting almost $1 \%$ of the world's population (1). Currently, there is no magnetic resonance (MR) imaging finding specific to or strongly suggestive of schizophrenia.

Understanding the neural network hypothetically responsible for the pathogenesis of schizophrenia requires a precise determination of the extent and distribution of abnormalities in brain anatomy and function (2). Functional imaging has been informative for understanding individual symptoms: Negative symptoms correlate with decreased activity in the dorsolateral prefrontal cortex $(3,4)$, and auditory hallucinations correlate with an increased blood flow in the Broca area in the left hemisphere (5).

The use of brain activation maps to detect brain areas with a different functional response is heavily dependent on the stimulus itself. Although a wide range of paradigms, including different senses (mainly visual but also olfactory) with distinctive features for each paradigm, has been used to study the emotional response with functional MR imaging, most of the studies in psychoses have used the visual sensory modality through the recognition of facial emotions (6-9). However, language impairment is one of the "core" phenomenological characteristics of patients with

\section{Advances in Knowledge}

- Functional MR imaging abnormalities associated with auditory emotional stimuli coexist with focal brain density reductions in schizophrenic patients experiencing chronic auditory hallucinations.

- Large coinciding brain clusters were found in the left and right middle temporal and superior temporal gyri; smaller coinciding clusters were found in the left posterior and right anterior cingular gyri, left inferior frontal gyrus, and middle occipital gyrus; these areas are related to the abnormal neural network involved in the auditory emotional dysfunction seen in these patients. schizophrenia, emphasizing the depth of the evaluation, so auditory stimuli with and without emotional charge seem more adequate in this context. Furthermore, schizophrenic patients have a reduction in size in different cortical areas, as demonstrated with the morphologic analysis of T1-weighted MR images (10).

Although many brain regions have been implicated in schizophrenia by means of MR, no single region has been consistently reported as abnormal to date. Differences between samples (clinical heterogeneity) and in methods among studies (sequences, paradigm, and postprocessing heterogeneity) may explain the lack of consistency in the results obtained in this field. Developing a clear understanding of the pathologic details of specific schizophrenic phenotypes is one of the greatest challenges in psychiatry. The purpose of our study was to prospectively evaluate if MR functional abnormalities associated with auditory emotional stimuli coexist with focal brain reductions in schizophrenic patients with chronic auditory hallucinations.

\section{Materials and Methods}

\section{Patient and Control Participant}

\section{Characteristics}

All patients and control participants gave written informed consent to participate in the research, which was approved by the local ethics committee. The ability of psychiatric patients to provide informed consent to participate in clinical research applies more to patients with conceptual disorganization and poor attention than to those experiencing mainly hallucinations (11). All patients in our series were experiencing hallucinations; however, all participants could read and understand the consent form, none were admitted to the hospital or underwent outpatient commitment at the time, and all were legally competent.

A group of 21 right-handed male patients with schizophrenia and persistent hallucinations (according to the Diagnostic and Statistical Manual of Mental Disorders, fourth edition) were studied.
Right-handed men were selected to avoid bias due to differences in brain structure and because schizophrenia affects men and women differently. Their ages ranged from 21 to 51 years (mean \pm standard deviation, 39 years \pm 10); all had a secondary school education level. These patients were selected from a sample of 160 patients with auditory hallucinations confirmed by their psychiatrist and clinical assessment (12).

All 21 patients in our series met the following selection criteria for persistent hallucinations: persistence of hallucinations despite pharmacologic treatment was present in all patients. Two antipsychotic drugs were tried at doses equivalent to at least $600 \mathrm{mg} / \mathrm{d}$ of chlorpromazine in the last year, but the voices heard were not modified in any way by treatment and were present at least once per day in the past year. All patients were being treated with antipsychotic medications at time of evaluation, 16 with second-generation and five with combined first- and second-generation antipsychotic drugs. The duration of treatment was 14.3 years \pm 6.9 . All patients heard voices during the functional MR data acquisition.

Schizophrenic patients began to hear hallucinations at a mean age of 23 years \pm 10 (range, $15-43$ ). The mean duration of illness was 15 years \pm 8 . All patients were clinically assessed by two

\section{Published online \\ 10.1148/radiol.2442060727}

Radiology 2007; 244:549-556

\section{Abbreviations:}

$3 \mathrm{D}=$ three-dimensional VBM $=$ voxel-based morphometry

\section{Author contributions:}

Guarantors of integrity of entire study, L.M., M.R., J.S. study concepts/study design or data acquisition or data analysis/interpretation, all authors; manuscript drafting or manuscript revision for important intellectual content, all authors; approval of final version of submitted manuscript, all authors; literature research, L.M., J.J.L., G.G., M.R., J.S.; clinical studies, L.M., J.J.L., E.J.A., C.P., J.S.; experimental studies, L.M., J.J.L., G.G., D.M., J.S.; statistical analysis, L.M., J.J.L., G.G.; and manuscript editing, L.M., J.J.L., G.G., C.P., M.R., J.S.

Authors stated no financial relationship to disclose. 
of the authors (E.J.A. and J.S., each with more than 16 years experience in clinical psychiatry) by consensus. Scores from both the Positive and Negative Syndrome Scale (13) and Psychotic Symptom Rating Scales were obtained during the 24-hour period before MR examination (14).

Ten healthy control participants were selected by matching the schizophrenic patients with respect to ethnic group (white), age (35 years \pm 7 for controls vs 39 years \pm 10 for patients), and education level (secondary school). Control participants were also righthanded men. None of them had a personal or family history of mental disorders or perceptual abnormalities, as assessed with a brief mental health questionnaire. No individual in either group had from hearing loss.

\section{MR Acquisition}

Both functional and morphologic MR images were acquired with a $1.5-\mathrm{T}$ scanner (Intera; Philips Medical Systems, Best, the Netherlands) with a quadrature volume head coil. The imaging plane was transverse and oriented parallel to the inferior limit of the rostrum and the genu of the corpus callosum.

The functional MR images were obtained by means of blood oxygen level dependent contrast material-enhancement. An emotional auditory stimulation-response paradigm was designed to replicate those emotions related to the patient's hallucinatory experiences (15). Words were grouped into two classes, one with highly emotional and the other with neutral content, and recorded on a compact disc. The same set of emotional and neutral Spanish words was used for each patient. Given that the stimuli pattern of functional MR lasts 20 seconds for each block, the most relevant 13 words were selected. The emotional words were defined according to the words most frequently heard by psychotic patients with auditory hallucinations. A total of 65 words were chosen based on their frequency and meaning. The words were classified according to the qualitative analysis of their content in five categories: negative content and imperative tone, insult, imperative tone, exclamation related to emotional state, and positive content.
Neutral words were selected from a Spanish emotional valence database (15). At the end of the trial every participant was asked to score their level of anxiety. Emotional and neutral words were signif icantly different in both groups as shown by using a paired Student $t$ test $(P=$ .001 )

Patients had earphones adjusted to their heads and connected by a pair of air tubes to an external audio compact disc player. All participants underwent two functional MR studies, one session with the high-emotion and the other with the neutral-emotion content words. Neutral and emotional content session were randomly presented to avoid habituation confounding effects. For each session, the voice stimuli consisted of four periods of activation alternated with four periods of rest with a block design. Both sessions were separated by no less than 40 seconds.

A dynamic echo-planar $\mathrm{T}^{*}$-weighted functional MR sequence (repetition time msec/echo time msec, 2000/50; section thickness, $5 \mathrm{~mm}$, with no intersection gap; matrix, $96 \times 128$; field of view, 220 $\mathrm{mm}$; flip angle, $65^{\circ}$ ) was obtained in each session. The pixel size was $3.27 \times 1.72 \times$ $5 \mathrm{~mm}$, with a voxel volume of $28.12 \mathrm{~mm}^{3}$. Each dynamic acquisition consisted of 24 contiguous sections covering the whole brain. The emotional and neutral functional MR session order was randomized for both patients and controls

For the morphologic analysis, a high spatial resolution three-dimensional (3D) spoiled gradient-echo T1-weighted MR sequence (96 partitions, 7/1.9; section thickness, $1.25 \mathrm{~mm}$, with no intersection gap; matrix, $256 \times 256$; field of view, 220 $\mathrm{mm}$; flip angle, $8^{\circ}$ ) was acquired after the functional MR examinations. These anatomic images of the whole brain had voxel size of $0.86 \times 0.86 \times 1.25 \mathrm{~mm}$ giving a voxel volume of $0.9245 \mathrm{~mm}^{3}$.

During the acquisition, patients were under direct observation by psychiatrists and interviewed about their experiences immediately after the MR procedure.

\section{Postprocessing Analysis}

Whole-brain postprocessing analysis, including functional MR, voxel-based morphometry (VBM), and maps of coin cidence, was performed jointly by three of the authors (J.J.L., G.G., and L.M. more than 5 years experience in MR postprocessing each).

Functional MR methods.-MR images were realigned with a subvoxel movement correction to avoid spurious signals (16). Functional and morphologic images were coregistered and transformed into a standard space named MNI152 (Montreal Neurological Institute, Montreal, Canada). Voxels were resampled to $2 \times 2 \times 2 \mathrm{~mm}$ and spatially smoothed with a $3 \mathrm{D}$ Gaussian $6 \times 6 \times 6$-mm kernel filter to increase the signal-to-noise ratio, reduce the anatomic variability between participants, and approximate the voxel distribution to the normal distribution (17).

SPM2 (Statistical Parametric Mapping, Functional Imaging Laboratory, London, England) analysis was performed for each patient and control participant and by comparing groups. In the individual analysis, a design matrix was defined for each participant. Both an ideal hemodynamic response function and the mean value of each func tional MR session were included in the design matrix.

Images of subtraction between emotional and nonemotional content word (both against the rest task) were then extracted for every schizophrenic patient and control subject. These images were considered to be maps of emotional activation associated with the auditory response. A paired sample $t$ test map was calculated by using the control participants and patients to test the differences in activation between emotional and nonemotional sessions. Tests were performed by means of the general linear model (16), applying a random effects analysis that accounts for within- and between-subject differences. Common features were extracted by using the general linear model over the subtraction of contrasts.

VBM method.-SPM2 was used to perform the structural image processing and the comparison analyses. Statistically significant anatomical differences in gray matter volume among patients and control volunteers were explored 
by means of optimized VBM (18), which involves using specific gray matter templates to warp the gray matter segmented maps. Although volume loss is a more familiar term to radiologists, we will use gray matter density because neuropathlogic studies in schizophrenia have found not neuronal loss or gliosis, but smaller neurons, dendrites with a lower density of spines, and less extensive arborization (19).

Custom templates were created to avoid the presence of errors due to differences in the contrast of the images and to specific nonuniformities of each MR acquisition and demographic differences in the sample. The process in volved the translation of each image into the same Talairach stereotactic space, applying a 12-parameter affine transformation by using the standard MNI 152 (Montreal Neurological Institute) template as a reference. The normalized images were averaged and smoothed by using a 3D $8 \mathrm{~mm}^{3}$ Gaussian kernel to obtain the custom template used in the next step. In addition, a priori gray matter, white matter, and cerebrospinal fluid probabilistic maps were obtained at this stage by averaging and smoothing the segmentation output of normalized images.

The VBM process started with the normalization of the T1-weighted images (with affine functions) to the selfgenerated template, obtaining a set of images in the same stereotaxic space, and applying a trilinear interpolation with a final voxel size of $1 \times 1 \times 1 \mathrm{~mm}$. These images were segmented into gray matter, white matter, and cerebrospinal fluid. In addition, a cleaning process was performed, removing nonbrain tissue such as scalp, skull, and dural venous sinus (20). Estimation parameters from nonlinear spatial normalization (21) between segmented images and a priori probabilistic maps were created and employed to reconstruct a normalized version in MNI space of the original T1-weighted images. Finally, warped T1 images were segmented by obtaining gray matter maps, which were smoothed by using a $12 \times 12 \times 12 \mathrm{~mm} 3 \mathrm{D}$ Gaussian filter.

A parametric map was created to help show the differences between schizophrenic patients and control subjects for the gray matter in the whole brain.

Functional MR and VBM: Maps of coincidence.-Activation clusters identified on the functional MR parametric map with an uncorrected $P$ of less than 005 uncorrected were selected with a small extent threshold $(\kappa>5)$. Differences in structural images were given a threshold of $P$ less than .005 corrected for multiple comparisons and a large extent threshold ( $\kappa>200)$. Both threshold maps were overlaid to depict the common differences found by using both techniques.

The coincidence map was then generated voxel-by-voxel by multiplying the emotional subtracted functional MR images by the gray matter concentration differences. Both contrasts are defined as positive since both represent $t$ values. The sign of the product was maintained. The coincidence analysis showed high activity in low gray matter density areas: the higher the activity, the higher the $t$ value; the lower the density, the higher the $t$ value. Therefore, the higher activation area and concentration decrease allowed more highlighted area to appear. Clusters of coincidence were grouped into large ( $\geq 100$ voxels) and small ( $<100$ voxels) clusters; larger clusters are probably more relevant to the phenomenologic analysis.

To allow the direct overlay of the different data sets, both functional and morphometric MR images were normalized to the same space by means of the MNI template and coordinates, which are also in the Talairach space. VBM volumes were normalized to the MNI space by creating a self-generated template to avoid segmentation artifacts.

Functional MR and VBM need different assumptions to minimize important missing findings. Functional MR imaging techniques indirectly measure brain activity. Both individual emotional and neutral versus rest responses are comparisons measuring emotional and neutral content words related to activation. To detect group differences between emotional and neutral words, a new comparison was generated by test- ing emotional as larger than the neutral comparison. VBM technique does not test for individual intrasubject variations but for between-subject variations instead. Our approach to functional, morphometric, and coincidence multiparametric comparison analysis was designed to select only those differentiated areas between patients and control subjects under the particular paradigm.

\section{Statistical Analysis}

Both functional MR and VBM statistical measurements were made under the general linear model framework and $t$ tests were carried out to acquire the difference maps. Additionally, random effects analyses were applied to obtain functional maps. The statistical model included a group condition (patient vs control) and a covariate of interest (age). Since age is known to be a cause of change in brain structure $(20,22)$, a regression study was performed to evaluate the relationship between this effect and the amount of gray matter.

A regression study showed linear correlation between age and brain tissue volume. Therefore, age was modeled in order to minimize its effect on the results maps.

Statistical parametric maps were acquired by performing independent $t$ tests for each voxel across groups, using SPM2 one-tailed comparisons to measure the gray matter difference (patients $<$ controls) and minimize the age effect in probabilistic maps by using analysis of covariance. A $P$ of less than .005 was used to establish a significance threshold and a correction for multiple comparisons by using the false discovery rate method (23). Additionally, a cluster filtering $(\kappa)$ was applied only for reporting clusters with 200 or more voxels.

Anatomic areas that showed changes among study groups were labeled with the Talairach coordinates and Broadman areas by using Automated Area Labeling software (Cyceron; Centre d'Imagerie Cerebrale et de Recherche en Neurosciences, Paris, France) (24). Coordinates for identifying each area were determined by using the maximum $t$ value in the corresponding area. 


\section{Results}

\section{Clinical Evaluation}

The Positive and Negative Syndrome Scale mean score was 71 (range, 53-94; standard deviation $[\mathrm{SD}], 10)$ while the Psychic Symptom Rating Scale mean score was 30 (range, 24-36; SD, 4), reflecting a homogeneous sample of schizophrenic treatment-resistant patients with persistent auditory hallucinations.

\section{MR Evaluation}

MR image analysis revealed that the most relevant areas with an increase in the auditory-triggered emotional activation were (Talairach coordinates $\mathrm{x}, \mathrm{y}$, and $\mathrm{z}$, in millimeters) the right temporal middle $(52,2,-18 ; t=7.59)$, left temporal middle $(-60,-48,6 ; t=6.03)$, right superior temporal and Heschl (54, $-18,8 ; t=5.35)$, right superomedial frontal $(2,50,34 ; t=5.36)$, right angular $(44,-66,28 ; t=5.63)$, right posterior cingulum $(10,-36,28 ; t=5.44)$, left middle cingulum $(-8,-14,44 ; t=$ $5.31)$, and right thalamus $(4,-12,4$; $t=4.89$ ).

The morphometric analysis showed a main local density reduction in the left insula $(-42,16,-9 ; t=7.19)$, right lingual $(13,-45,-2 ; t=7.02)$, left postcentral $(-60,-11,24 ; t=6.82)$, right precuneus $(12,-50,5 ; t=6.52)$, right insula $(47,15,-6 ; t=6.52)$, right superomedial frontal $(10,59,-1 ; t=$ $6.38)$, left lingual $(-11,-52,1 ; t=$ $6.29)$, and left middle temporal $(-62$, $-62,-3 ; t=6.17$ )

\section{Coincidence Map Evaluation}

The coincidence analysis selected different regions (Table) and showed that the regions had either large or small clusters of both emotional activation and volume loss (Figure). As shown, larger clusters were found in the left and right middle temporal and left and right superior temporal gyri. Smaller clusters were found in the left posterior and right anterior cingular gyri, left inferior opercular frontal gyrus, and right middle occipital gyrus.

\section{Discussion}

A recent meta-analysis of 15 VBM studies found that the left superior temporal gyrus and the left medial temporal lobe are the key regions of structural difference between patients with schizophrenia and control subjects (10). The human voice contains in its acoustic structure information on the speaker's emotional state which is easily perceived with remarkable accuracy. The neural basis of the perception of speaker-related vocal features was found bilaterally in the upper bank of the superior temporal sulcus (25)

The coexistence of functional and morphologic abnormalities behaves as a filter selecting areas that could be specifically related to the schizophrenic phenotype under study. Our data confirm the left middle temporal gyrus as the most important structural and functional area implicated in the pathogenesis of auditory hallucinations.

Auditory hallucinations activate the left and right superior and middle temporal gyri. Structures activated during the perception of external voices are also activated during auditory hallucinations with the additional activation found in areas responsible for the processing of emotion (26). A clear overactivation of the temporal cortex, frontal lobe, insula, cingulate, and amygdala was found in patients when hearing emotional words compared with control subjects. However, when studying the effect of the auditory-triggered emotion on schizophrenic patients with chronic auditory hallucinations, an overactivation of both middle temporal, right superior temporal, and Heschl gyri; left frontal superomedial and right angular; cingulum; and thalamus is depicted. This overactivation of the limbic and frontal brain areas in persistent hallucinatory patients using a specific auditory emotional paradigm may partially reflect the dysfunction of the emotional response to auditory stimuli in schizophrenia. Some of these activated areas may reflect a true hemodynamic abnormality related to functional changes, while others may express the adoption of a modified strategy to perform adequately by using different cognitive skills and engaging different brain regions. The coexistence of gray matter deficits with activated areas may differentiate a true abnormality from an adopted strategy.

Voxel-based gray matter reduction implies a decrease in the optical density of a region. Although no neural loss has been found in patients with schizophrenia, a decreased cortical volume due to smaller neurons and dendrites with a lower density of spines and less extensive arborization is observed in pathologic studies (19). Postmortem studies in patients with schizophrenia have shown a substantial frontal gray matter volume reduction when compared with 


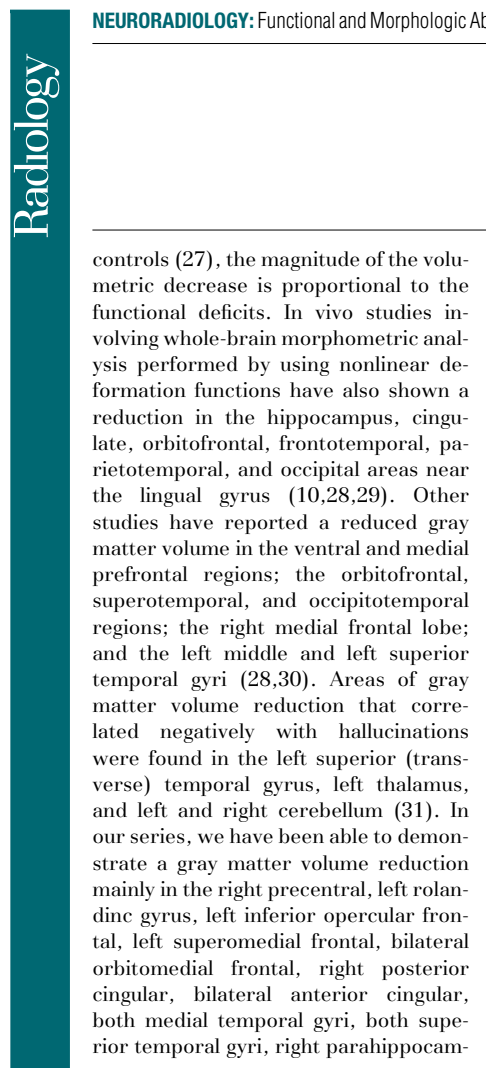

pus, right insula, and right precuneum regions.

The ventromedial prefrontal cortex and the amygdala are involved in the normal processing of emotional signals. The temporolimbic and fronto-orbital network have also been implicated in regulating emotion, and the medial limbic system, specifically, has been related to the emotion-processing deficits of schizophrenia. The lingual gyrus at the inferior occipital region was observed to be reduced in these patients (28). Although the reduced volumes in the temporal regions were more marked on the left side (28), we observed a quasi-symmetrical pattern of neuronal reduction. Our different depiction of abnormalities may be related to differences in the phenomenology of schizophrenia studied, the homogeneous series evaluated, the whole-brain morphometric approach, and the appropriate analysis of the confounders (ie, age, gender, handedness and education level).

The VBM approach provides details regarding the specific points of maximal density change. However, many of the morphologic structural abnormalities are relatively subtle and have pronounced interindividual variability, making their use as diagnostic markers of schizophrenia less sensitive.

A neurophysiologic interaction among psychopathology (auditory hallucinations), brain function (increased hemodynamic response in the temporal lobe) and structure (gray matter deficits) has been previously hypothesized (26). It is generally accepted that areas of decreased perfusion parallel decreases in gray matter concentration. An example of this is the age-related brain reduction most probably associated with a decrease in blood flow and metabolism in those areas (32). However, our findings show that in schizophrenic patients abnormal activation may be found in specific areas of maximal neural density decrement. These areas of coincidence where the same voxels have hemodynamic functional changes associated with the emotional auditory-triggered response and focal decreased volume could possibly express a compensation phenomenon in which regions with decreased volume need a larger hemodynamic dysfunctional response to a defined paradigm.

The different areas found in the results of the coincidence maps have rela-

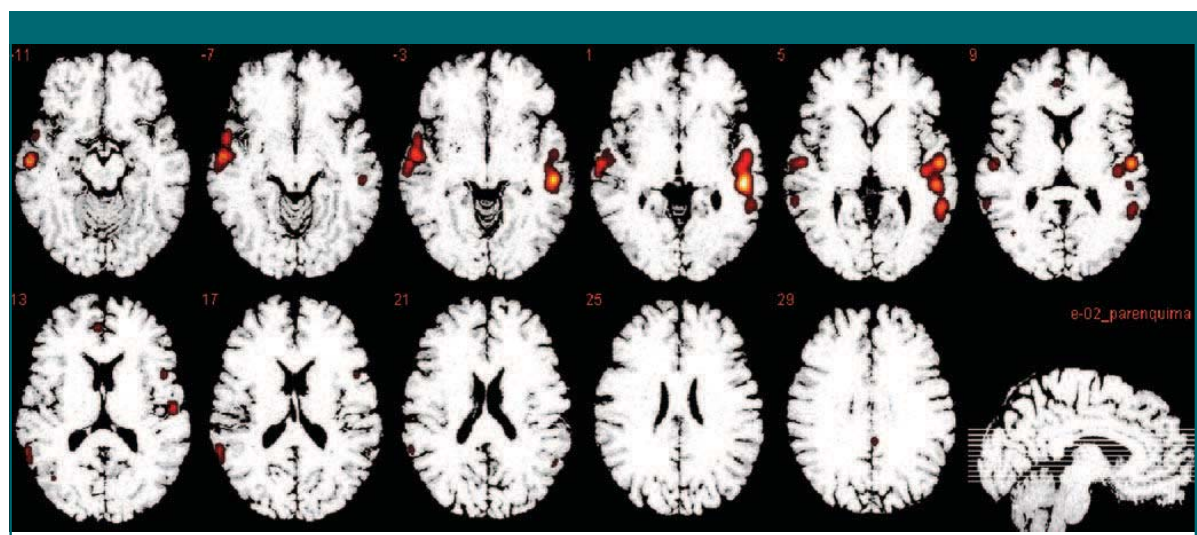

Functional MR coincidence maps. Highlighted areas indicate increased activation associated with emotional auditory stimuli and decreased gray matter volume. Yellow areas represent greater values for functional and morphometric statistical source images. Selected clusters can appear in white matter by means of partial volume effects after spatial smoothing. 
tionships with both emotion and schizophrenia, and are probably part of the auditory hallucination phenotype. The bilateral middle temporal gyri are also being involved in emotion (33). The right superior temporal gyrus is mainly involved with hearing, emotion, and changes in the aural sensory environment $(34,35)$. The posterior cingular gyrus is involved with the association of recognized words (36), the threat of emotional words, and unpleasant words (37). The anterior cingular gyrus is involved with attention, while the opercular frontal gyrus has traditionally been affected in schizophrenic patients (orbital prefrontal cortex) and involved with decision-making (38).

The study had study limitations. As all patients had auditory hallucinations at MR, a subtraction strategy was developed to depict only those voxels activated during the emotional stimuli but not from the neutral voices. This way, emotion-related auditory activation areas can be better delineated.

The voxel size of the functional and morphometric MR images was approximately 30 times larger than the functional voxel $\left(28.12\right.$ vs $\left.0.9245 \mathrm{~mm}^{3}\right)$. Since partial volume effect may exist, it is possible that one functional MR voxel will not be activated while part of it is depicted during the morphometric analysis. This means that our approach is conservative, depicting only the coincidence voxels at the price of losing small contributions, making the results easily reproducible. Changes in the volume and activation of these brain areas can result from the medication, not just the symptoms, in schizophrenic patients. However, smaller volumes of gray matter in similar regions as those observed in this study have also been found in neuroleptic-naive patients and in firstepisode schizophrenic patients, thus giving more relevance to the disease than to medication $(9,29)$.

As schizophrenia affects men and women differently, the selected population consisted only of men to avoid this bias in the analysis of the temporolimbic structures (39). Also, factors of ethnicity, gender, hand dominance, age, and education level were minimized. We recognize that the results obtained from a defined homogeneous sample, only including schizophrenic patients with chronic resistant auditory hallucinations, can not be generalized to schizophrenia as a whole. The clinical heterogeneity of schizophrenia is beyond the scope of this paper.

Also, we have used a 1.5-T imager with high $(>99 \%)$ mean specificity in our study, and we recognize that field magnets with higher specificity will probably produce better results by increasing the signal-to-noise and reproducibility in the functional MR study. This also allows a decrease in the voxel size (40). These improvements deserve further evaluation.

In summary, coincidence analysis grouping functional MR abnormalities with focal brain density reductions in schizophrenic patients with chronic auditory hallucinations are largely depicted at the middle, superior temporal, and cingular gyri. The possibility that coincidence maps could be used for follow-up of these patients after treatment should be evaluated.

\section{References}

1. Buchanan RW, Carpenter WT Jr. Schizophrenia and other psychotic disorders. In Sadock BJ, Sadock VA, eds. Kaplan \& Sadock's comprehensive textbook of psychiatry. 8th ed. Philadelphia, Pa: Lippincott Williams \& Wilkins, 2005; 1329-1558.

2. Honey GD, Sharma T, Suckling J, et al. The functional neuroanatomy of schizophrenic subsyndromes. Psychol Med 2003;33:10071018.

3. Weinberger DR, Berman KF. Prefrontal function in schizophrenia: confounds and controversies. Philos Trans R Soc Lond B Biol Sci 1996;351:1495-1503.

4. Semkovska M, Bedard MA, Stip E. Hypofrontality and negative symptoms in schizophrenia: synthesis of anatomic and neuropsychological knowledge and ecological perspectives [in French]. Encephale 2001;27 405-415.

5. McGuire PK, Shah GM, Murray RM. Increased blood flow in Broca's area during auditory hallucinations in schizophrenia. Lancet 1993;342:703-706.

6. Schneider F, Weiss U, Kessler C, et al. Differential amygdala activation in schizophre- nia during sadness. Schizophr Res 1998;34 133-142.

7. Phillips ML, Williams L, Senior C, et al. A differential neural response to threatening and non-threatening negative facial expressions in paranoid and non-paranoid schizophrenics. Psychiatry Res 1999;92:11-31.

8. Kosaka H, Omori M, Murata T, et al. Differential amygdala response during facial recognition in patients with schizophrenia: an fMRI study. Schizophr Res 2002;57:87-95.

9. Gur RE, McGrath C, Chan RM, et al. An fMRI study of facial emotion processing in patients with schizophrenia. Am J Psychiatry 2002;159:1992-1999.

10. Honea R, Crow TJ, Passingham D, Mackay CE. Regional deficits in brain volume in schizophrenia: a meta-analysis of voxelbased morphometry studies. Am J Psychiatry 2005; 162:2233-2245.

11. Howe V, Foister K, Jenkins K, Skene L, Copolov D, Keks N. Competence to give informed consent in acute psychosis is associated with symptoms rather than diagnosis. Schizophr Res 2005;77:211-214.

12. Gonzalez JC, Aguilar EJ, Berenguer V, Leal C, Sanjuan J. Persistent auditory hallucinations. Psychopathology 2006;39:120-125.

13. Kay SR, Fiszbein A, Opler LA. The Positive and Negative Syndrome Scale (PANSS) for schizophrenia. Schizophr Bull 1987;13:261276.

14. Haddock G, McCarron J, Tarrier N, Faragher EB. Scales to measure dimensions of hallucinations and delusions: the psychotic symptom rating scales (PSYRATS). Psychol Med 1999;29:879-888.

15. Sanjuan J, Lull JJ, Martí-Bonmatí L, et al. Emotional auditory paradigm in neuroimaging: a base for the study of psychosis. Actas Esp Psiquiatr 2005;33:383-389.

16. Friston KJ, Holmes AP, Worsley KJ, Poline JP, Frith CD, Frackowiak RS. Statistical parametric maps in functional imaging: a general linear approach. Hum Brain Mapp $1995 ; 2: 189-210$.

17. Worsley KJ, Liao CH, Aston J, et al. A general statistical analysis for fMRI data. Neuroimage 2002;15:1-15.

18. Ashburner J, Friston KJ. Voxel-based morphometry: the methods. Neuroimage 2000;11:805-821.

19. Harrison PJ. The neuropathology of schizophrenia. A critical review of the data and their interpretation. Brain 1999;122:593624 .

20. Good CD, Johnsrude IS, Ashburner J, Henson RN, Friston KJ, Frackowiak RS. A voxel- 
based morphometric study of aging in 465 normal adult human brains. Neuroimage 2001;14:21-36.

21. Ashburner J, Friston KJ. Nonlinear spatial normalization using basis functions. Hum Brain Mapp 1999;7:254-266.

22. Coffey CE, Lucke JF, Saxton JA, et al. Sex differences in brain aging: a quantitative magnetic resonance imaging study. Arch Neurol 1998;55:169-179.

23. Genovese CR, Lazar NA, Nichols T. Thresh olding of statistical maps in functional neuroimaging using the false discovery rate. Neuroimage 2002;15:870-878

24. Tzourio-Mazoyer N, Landeau B, Papathanassiou D, et al. Automated anatomical labeling of activations in SPM using a macroscopic anatomical parcellation of the MNI MRI single subject brain. Neuroimage 2002;15:273289.

25. Belin P, Zatorre RJ, Lafaille P, Ahad P, Pike B. Voice-selective areas in human auditory cortex. Nature 2000;403:309-312.

26. Kircher TT, Thienel R. Functional brain im aging of symptoms and cognition in schizophrenia. Prog Brain Res 2005;150:299-308.

27. Selemon LD, Kleinman JE, Herman MM, Goldman-Rakic PS. Smaller frontal gray matter volume in postmortem schizophrenic brains. Am J Psychiatry 2002;159:19831991
28. Davatzikos C, Shen D, Gur RC, et al. Wholebrain morphometric study of schizophrenia revealing a spatially complex set of focal abnormalities. Arch Gen Psychiatry 2005;62: 1218-1227.

29. Job DE, Whalley HC, McConnell S, Glabus M, Johnstone EC, Lawrie SM. Structural gray matter differences between first-episode schizophrenics and normal controls using voxel-based morphometry. Neuroimage $2002 ; 17: 880-889$.

30. Gaser C, Nenadic I, Volz HP, Buchel C, Sauer H. Neuroanatomy of "hearing voices": a frontotemporal brain structural abnormality associated with auditory hallucinations in schizophrenia. Cereb Cortex 2004;14:91-

31. Neckelmann G, Specht K, Lund A, et al. MP morphometry analysis of grey matter volume reduction in schizophrenia: association with hallucinations. Int J Neurosci 2006;116:923.

32. Van Laere KJ, Dierckx RA. Brain perfusion SPECT: age- and sex-related effects correlated with voxel-based morphometric findings in healthy adults. Radiology $2001 ; 221$ $810-817$

33. Phillips ML, Bullmore ET, Howard R, et al. Investigation of facial recognition memory and happy and sad facial expression perception: an fMRI study. Psychiatry Res perception: an fM
34. Aalto S, Naatanen P, Wallius E, et al. Neuroanatomical substrata of amusement and sadness: a PET activation study using film stimuli. NeuroReport 2002;13:67-73.

35. Downar J, Crawley AP, Mikulis DJ, Davis KD. A multimodal cortical network for the detection of changes in the sensory environment. Nat Neurosci 2000;3:277-283.

36. Maddock RJ, Buonocore MH. Activation of left posterior cingulate gyrus by the auditory presentation of threat-related words: an fMRI study. Psychiatry Res 1997;75:1-14.

37. Hunkin NM, Mayes AR, Gregory LJ, et al. Novelty-related activation within the medial temporal lobes. Neuropsychologia 2002;40: 1456-1464.

38. Rogers RD, Owen AM, Middleton HC, et al. Choosing between small, likely rewards and large, unlikely rewards activates inferior and orbital prefrontal cortex. J Neurosci 1999; 19:9029-9038.

39. Gur RE, Turetsky BI, Cowell PE, et al. Temporolimbic volume reductions in schizophreiia. Arch Gen Psychiatry 2000;57:769-775.

40. Zou KH, Greve DN, Wang M, et al. Reproducibility of functional MR imaging: preliminary results of prospective multi-institutional study performed by Biomedical Informatics Research Network. Radiology 2005;237: 781-789. 


\section{5.d. - Comment to the manuscript and addendum}

The objective of the article was the integration of two different viewpoints about schizophrenia. The integration of neuroimaging data was considered as valuable. In the case of the neuroimaging data from schizophrenic patients and healthy control subjects, some brain areas were detected as a start to study their function and structure in schizophrenic patients. However, in the applied methodology to obtain coincidence areas, some potential problems have not been taken into account. As presented in the manuscript, fMRI data and structural data were spatially normalized prior to the group comparison statistics performed on each type of data.

There are different approaches to spatial normalization, as brains differ largely in structure from subject to subject, even in the case of healthy subjects with similar characteristics (such as age, gender or total brain volume). Differences also exist at the microscopic level (Devlin and Poldrack, 2007). Spatial normalization applied in the study that is presented along the thesis included affine and nonlinear transformations of the images (Ashburner and Friston, 1999). Other authors propose to directly observe each subject's anatomy and manually observe correspondences between brain structures (Devlin and Poldrack, 2007); Dale (Dale et al, 1999) proposed to observe the cortical surface instead of observing the brain volume, by means of automated procedures; Van Essen also proposed to work on the subjects' cortical surface data instead of cortical volume data (Essen Van et al, 2001).

Automatic spatial normalization involves the possibility of incorrect registration between subjects' anatomy, that could lead to the extraction of wrong conclusions from the analysed data (in the case of VBM, see Bookstein, 2000). Results derived from spatially normalized data should be concisely reviewed and scrutiny of the data from each subject's activation or volume characteristics should be carried out (Ashburner, Friston, 2001). 
Results in this chapter could be biased because of mis-registration between subjects' anatomy, that could have led to systematic errors in both techniques. The objective of detecting areas of interest, with possible mis-function and volume differences in schizophrenic patients, is still considered of interest by the PhD candidate. The detected areas have been a start point for further analyses.

The following sentences were detected that contained mistakes in the manuscript (page numbers correspond to the manuscript):

p.552. There was a mistake in the Gaussian kernel size, that stated it was $8 \mathrm{~mm}^{3}$. It should be:

The normalized images were averaged and smoothed by using a 3D $8 \times 8 \times 8 \mathrm{~mm}$ Gaussian kernel to obtain the custom template used in the next step.

p.555 - The sentence that reads "The voxel size of the functional and ..." could be re-stated with the word volumes. It was also somewhat confusing.

Acquired voxel volumes were different in functional data $\left(28.12 \mathrm{~mm}^{3}\right)$, compared to morphometric data $\left(0.9425 \mathrm{~mm}^{3}\right)$. Voxel volumes that had been acquired by means of fMRI were approximately 30 times bigger than anatomical data acquired for the VBM procedure. 


\section{6. fMRI activation localization enhanced by Non Local Means filtering}

"Anyone who has never made a mistake has never tried anything new" Albert Einstein (1879-1955).

In this chapter, the NL-Means is introduced, in a manuscript that has already been sent for publication. In the article, the adaptation of a new filter named NL-Means to fMRI and its behaviour in the denoising of fMRI data are shown. Tests, both on synthetic and clinical data, are presented in the manuscript, demonstrating the improved localization power of the proposed method. The proposed method has been applied to the fMRI data with the auditory paradigm, in one of the subjects previously studied, as shown in the manuscript. The structure of this chapter introduces first the $\mathrm{PhD}$ candidate work in the manuscript. Afterwards the manuscript is presented; and finally supplementary results are shown that support new research guidelines.

The work presented in the manuscript consisted in adapting the NL-Means method to $\mathrm{fMRI}$ and testing the NL-Means filter on $\mathrm{fMRI}$ real and synthetic data. The adaptation of the NL-Means was generated in C code for Matlab, based on the implementation by J.V. Manjón for structural images (Manjón et al, in press). The implemented method was firstly optimized so the optimal filtering parameters were selected. This included the testing of many possible selections of noise level control and sizes of the search and the similarity windows. The optimization started with the parameters chosen by Manjón et al for structural MR images, in synthetic data. 
The NL-Means filtering method showed, both in clinical and real fMRI data, a very high performance in the denoising process. Thus, studies about inter-subject analysis with NL-Means denoised data will be introduced.

\section{6.a. - PhD candidate contributions}

The tasks carried out by the $\mathrm{PhD}$ candidate in the elaboration of the manuscript were:

- Writing of the manuscript, all sections. Figure generation.

- Literature research.

- Software phantom generation.

- Adaptation of the proposed filtering method to fMRI.

- Theoretical conception.

- Experimental tests on the clinical and the synthetic datasets.

- Editing and supervision of the manuscript, in all sections.

\section{fMRI activation localization enhanced by Non Local Means filtering}

Juan J. Lull, José V. Manjón, Luis Martí-Bonmatí, Gracián GarcíaMartí, Julio Sanjuán, Nuria Lull, María A de la Iglesia, Montserrat Robles. 


\section{fMRI activation localization enhanced by NL- means filtering}

Juan-J. Lull ${ }^{a, *}$, José V. Manjón ${ }^{a}$, Luis Martí-Bonmatíl ${ }^{b, c}$, Gracián García-Martí ${ }^{a, c}$, Julio Sanjuán ${ }^{\mathrm{d}}$, Nuria Lull ${ }^{\mathrm{a}, \mathrm{e}}$, Maria A de la Iglesia ${ }^{\mathrm{a}}$, Montserrat Robles ${ }^{\mathrm{a}}$

a Biomedical Informatics Group, IBIME. ITACA Institute. Universidad Politécnica de Valencia. Edificio 8G. Camino de Vera s/n. 46022 Valencia, Spain.

${ }^{b}$ Department of Radiology. Hospital Universitario Dr. Peset. Valencia, Spain.

${ }^{\mathrm{C}}$ Radiology Department, Hospital Quirón, Valencia, Spain.

'Psychiatric Unit, Faculty of Medicine, Clinical Hospital, University of Valencia, Spain.

${ }^{\text {e}} \mathrm{CEU}-U \mathrm{CH}$ Universidad Cardenal Herrera Valencia, Spain.

Short title: fMRI localization enhanced by NL-means filtering

${ }^{*}$ Corresponding author. Biomedical Informatics Group, IBIME. ITACA Institute. Universidad Politécnica de Valencia. Edificio 8G. Camino de Vera s/n. 46022 Valencia. Spain.

Telephone: +34 963877000 ext.75275

Fax: + 34963877279

E-mail address: jualulno@upv.es 


\section{ABSTRACT}

One of the main problems in Functional Magnetic Resonance Imaging (fMRI) is that the signal amplitude from activated voxels is similar to the amplitude of the random noise naturally present in the images. Weakly activated voxels may therefore pass statistical tests undetected. Typically, the Gaussian filtering method is applied to restore the fMRI signal as part of the pre-processing used before statistical analysis of the data. This filter increases the functional signal at the expense of a sub-optimal signal enhancement and a reduction in resolution and accuracy in spatial localization. Recently, advanced filtering methods have been proposed for reducing noise from the fMRI data. In the present work, a new method for restoring fMRI signals is presented which is able to reduce random noise without affecting significantly the original image resolution. The proposed method is based on the Non-Local Means (NL-Means) filter. This paper addresses the problem of accurate spatial localization of brain activated areas, comparing the Gaussian Filter, the Adaptive Bilateral Filter and the NL-Means Filter using both synthetic and real fMRI data.

NL-Means is a special neighbourhood filter that reduces the noise in each voxel by averaging similar voxels non-locally, i.e. any region in the image can help denoising one particular region, not only nearby regions.

The results showed that the NL-Means method enhanced the fMRI signal, by decreasing the noise level, without introducing noticeable blurring effects, as demonstrated by its better specificity and sensitivity in detecting activated voxels, especially of brain activation in areas of bordering sharp transitions, compared to both the Gaussian Filter and the Adaptive Bilateral Filter. Due to the excellent behaviour of the NL-means filter we suggest its use as part of the pre-processing in fMRI studies.

Key words

fMRI; Image Quality Enhancement; Brain; Gaussian Distribution; noise filtering. 


\section{INTRODUCTION}

The application of the fMRI technique is becoming wider everyday. Its ability to detect changes in function in the healthy and unhealthy brain makes this non-invasive technique suitable to study brain activity in-vivo. Recent studies show that fMRI mapping can successfully locate the functional areas of interest preoperatively aiding in tumour resection operations. fMRI enables a better identification of the sensorimotor cortex by means of $\mathrm{fMRI}$ than traditional identification of the motor areas in MR anatomical images (Pujol et al, 2008); fMRI has proved a reliable measure of the language lateralization when language is to be preserved in tumour surgery (Ruff et al, 2008); it provides a high coincidence with intraoperative cortical stimulation in localizing the motor centers $(95 \%$ coincidence in the exact location; 33 patients; see Majos et al, 2005). Successful fMRI mapping can be obtained routinely in most patients with cerebral tumors (Vlieger et al, 2004). Clinical validation of the application of fMRI to tumour resection is currently being conducted (Geerts et al, 2007; Petrella et al, 2006).

Although fMRI is already being successfully applied in many studies of brain function, such as brain tumour pre-operative explorations, there have lately appeared issues related to the exact spatial localization of the fMRI activation areas, related to spatial smoothing (Reimold et al, 2006; Walker et al, 2006; Wink $A M$ et al, 2004). Some localization issues are directly related to the use of Gaussian smoothing, a process that is often applied to increase the Signal to Noise Ratio (SNR), which is inherently low in fMRI. Reimold et al. (2006) demonstrated that, in addition to the loss of resolution that is inherent in spatial smoothing, it can also lead to counterintuitive artifacts, such as a displacement of the detected peak of activation, which could lead to a spatially narrowing in a way that a region may be mistakenly identified as an additional finding.

Many reasons make the accurate detection of spatial activation areas necessary: Delimitation of functional areas surrounding tumors and other brain malformations must be precisely delineated to aid in the operative planning. Increasing spatial resolution of fMRI data is a critical topic nowadays (Bandettini, 
2007). Even though a higher resolution is being achieved in acquisition, typically used filters, such as spatial smoothing, reduce the resolution present in the fMRI data.

Alternative methods to Gaussian smoothing have been proposed for fMRI. The earliest one was proposed by Descombes et al. (1998). Descombes proposed to restore the fMRI data by using spatiotemporal Markov Random Fields (MRF). The method was based on the interaction between neighboring voxels. This method had several drawbacks: Even though MRFs do not smooth the fMRI data as much as spatial Gaussian filtering, the influence from the neighbours is critical. Therefore, if there was no good connection from one voxel to its neighbours (e.g. connection by one only voxel), then the activation would not be detected in that voxel. Besides, the influence of MRF on further statistics is not well known yet. One last drawback of the method proposed by Descombes is that temporal Gaussian filtering is not used at present. Instead, temporal correlations are treated by means of autoregressive models (Purdon, Weisskoff, 1998).

More recently, Solé et al. (2001) proposed to separate active voxels from non-active voxels iteratively by diffusion averaging. The method however needed some clearly distinguishable activated voxels. If the signal was low compared to noise throughout the whole brain, then the method would not be successful. The method also expected a periodicity in the signal, which is not always present, and assumed that all voxels activated with the same temporal pattern.

Multivariate approaches have also been explored through Independent Components Analysis (McKeown et al, 1998), Canonical Correlation Analysis (Friman et al, 2001) and Wavelets (Desco et al, 2001). These techniques have not been extensively applied to $\mathrm{fMRI}$ because their impact on subsequent statistical analyses is usually unclear (Petersson et al, 1999).

A number of hierarchical Bayesian models have been proposed (Gössl et al, 2001; Penny et al, 2005; Woolrich et al, 2004; Flandin, Penny, 2007), that model the noise instead of removing it from the fMRI data. Modeling the noise is 
computational intensive so the aforementioned studies also deal with diverse ways of speeding up the estimations. Woolrich (2004) and Penny and Flandin (2005, 2007) proposed a Variational Bayes framework, that sped up the original algorithms hundreds of times. However, the selection of spatial a priori probability values still remains problematic.

Kim et al. (2005) also proposed a method, the RADSPM, that would estimate the SPM from the noisy fMRI, compute the diffusion coefficients in the SPM space, and then perform the diffusion in the structural information-removed fMRI data using the coefficients previously computed (Kim et al, 2005). These steps were iterated until convergence. The processing time was the weak point of RADSPM, as it needed to compute a new SPM per iteration of the method, and tens of iterations were usually needed.

A method which could overcome such problems is the Non-Local Means (NL-Means) filter, an algorithm that was introduced by Buades et al. in 2005 (Buades et al, 2005). This method is a special kind of Neighbourhood filter that reduces the noise in every voxel by averaging its value with the values of other similar voxels around the specific voxel. Different filters have been proposed based on the same principle, i.e. the SUSAN Filter (Smith and Brady, 1997) and the Bilateral Filter (Tomasi and Manduchi, 1998), amongst others.

The Bilateral filter, which has shown to respect anatomical edges, is especially suited for detecting fMRI activations near brain tumour lesions, minimizing the blurring of apparent brain activity across anatomical boundaries and into regions of non-activation (Walker et al, 2006). However, such a filter does not perform optimally when noise level becomes significant.

The NL-Means filter introduces two important differences compared to other Neighbourhood filters: First, the similarity measure between voxels has been made robust. Second, spatial distance between voxels is not restricted to be local (thus, the method is non-local). By one hand, the similarity between two voxels $x$ and $y$ in NL-means is measured by the grey level differences in a whole Gaussian 
neighborhood of $x$ and $y$. By the other hand, the spatial distance between voxels, that was restricted to be local in other filtering schemes, needs no more to be local, as the robust measure of similarity lets the algorithm find distant voxels with similar neighbourhoods to the one being filtered. For example, a couple of pixels $x$ and $y$ such that their intensity levels are equal, can have a very small or nearly zero weight in the restoration of each other, since the configurations around $x$ and $y$ can be very different.

NL-means has excellent properties that could make the filter suitable for fMRI denoising. Firstly, its similarity measure is very robust to the noise power and therefore it is well suited to remove noise from typically low contrast activations. Secondly, the non-local means filter accomplishes the "noise-to-noise principle" which is not achieved by other methods, such as the Gaussian filter or other classical Neighbourhood filters. The noise-to-noise principle states that a denoising algorithm must transform a white noise image into a white noise image (with lower variance). Therefore, after the application of a filter the remaining noise should not change its nature. This is achieved by NL-Means, as demonstrated by Buades et al. (2007).

The NL-Means has been applied successfully to anatomical MRI (Coupé et al, 2007; Manjón et al, 2008), Diffusion-weighted and Diffusion-tensor MRI (WiestDasslé et al, 2007). The filter has also been applied to picture and movie denoising (Mahmoudi, Sapiro, 2005) and texture restoration (Brox, Cremers, 2007).

Our objective in this study was to test an optimized version of the NLMeans method adapted to fMRI filtering, in both real and synthetic fMRI data, in order to find out if this filter would improve the localization and detectability of functional patterns in fMRI studies. 


\section{MATERIAL AND METHODS}

To judge the influence of the NL-Means filter on the localization and detection of functional patterns in fMRI, it was compared with two methods typically used in this framework: Gaussian filtering (Friston et al, 2000) and the Adaptive Bilateral Filter (Walker et al, 2006). Other filtering methods were not introduced in the comparison, as they are more complex in nature and therefore comparisons results could be easily biased. Furthermore, proposed methods that have been presented in bibliography are usually compared to Gaussian filtering, so results from other filtering schemes could be compared with this one easily. The assessment was performed in both real and synthetic data.

\section{Gaussian Filter}

The Gaussian Filter consists of the convolution of a 3D image volume with a 3D Gaussian kernel. Usually, this Gaussian kernel is defined by its Full-WidthHalf-Maximum (FWHM), directly related to the parameter standard deviation, $\sigma$ :

$$
F W H M=\sigma \sqrt{8 \cdot \log (2)}
$$

Therefore, given an image volume I, the Gaussian filtered volume F is computed as:

$$
F_{p}=\sum_{\forall q \in S} I_{q} G(\sigma)_{p-q} \quad \forall p \in I
$$

Where $S$ is a 3D local region defined by $\sigma$, surrounding the processed voxel, $\mathrm{G}$ is a Gaussian kernel: 


$$
G(\sigma)_{p-q}=\frac{1}{2 \cdot \pi \cdot \sigma^{2}} e^{-\frac{(p-q)^{2}}{2 \sigma^{2}}}
$$

$p$ and $q$ represent different voxel coordinates in the volume.

\section{Adaptive Bilateral Filter}

The Bilateral Filter (Tomasi and Manduchi, 1998) is an extension of the Gaussian filter. It adds an edge-stopping function, such as Gauss, Tukey or Lorentz edge-stopping functions (Durand and Dorsey, 2002). The Bilateral Filter smoothes voxels that are near one another and that are similar in intensity, while preserving the data. Sharp changes in intensity are detected by means of the edge-stopping function.

$$
F_{p}=\sum_{\forall q \in S} I_{q} G(\sigma)_{p-q} G(\theta)_{I_{p}-I_{q}} \quad \forall p \in I
$$

In this function there are two Gaussian weighting factors defined by $\sigma$ and $\theta$, which are related to the proximity of the current voxel geometrically in the first case, and in intensity level in the second one. The first term $\left(G(\sigma)_{p-q}\right)$ is devoted to restrict locally the averaging process while the second term $\left(G(\theta)_{I_{p}-I_{q}}\right)$ adapts the weighting function to the underlying anatomical information. 


\section{NL-means filter}

The NL-Means filter, proposed by Buades et al. (Buades et al, 2005) is an evolution of the Neighbourhood filter (Yaroslavsky, 1985), which averages similar voxels according to their intensity distance. The main difference between the NLMeans and previous methods is that the similarity measure between voxels has been made more robust to the noise level by using a region comparison strategy rather than voxel comparison. Furthermore, the pattern redundancy is not restricted to be local (therefore, non-local). The NL-means filter can be applied in 3D (as it has been applied in MR anatomical image filtering) or, in the case of fMRI, 4D (along all acquired volumes), as the method accomplishes the noise to noise principle (it does not add serial correlations).

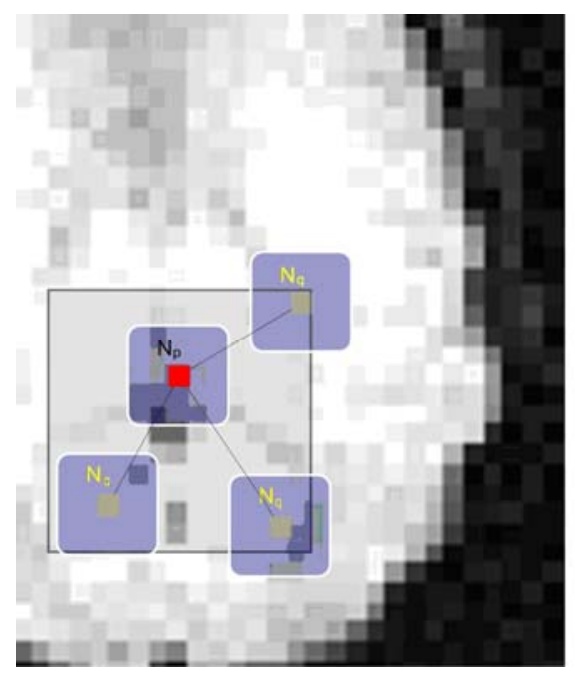

Figure 1. The NL-means principle. The voxel in restoration $p$ is characterised by its neighbourhood $\mathrm{N}(\mathrm{p})$, which is compared to every possible neighbourhood $(\mathrm{N}(\mathrm{q})$ ) in a bigger window around the voxel $p$, the Search Window.

The NL-means method is described below in more detail.

$$
\begin{aligned}
& F_{p}=\sum_{\forall q \in S} I_{q} w\left(N_{p}, N_{q}\right) \quad \forall p \in I \\
& 0 \leq w\left(N_{p}, N_{q}\right) \leq 1 \quad \sum_{\forall q \in S} w\left(N_{p}, N_{q}\right)=1
\end{aligned}
$$


where $F_{p}$ is the voxel being filtered and $I_{q}$ represents each other voxel in the image volume and $S$ is the Search Window around each voxel in restoration. The weights $\mathrm{w}\left(\mathrm{N}_{\mathrm{p}}, \mathrm{N}_{\mathrm{q}}\right)$ are based on the similarity between the neighbourhoods of the voxels in coordinates $\mathrm{p}$ and $\mathrm{q}\left(\mathrm{N}_{\mathrm{p}}\right.$ and $\mathrm{N}_{\mathrm{q}}$ ). In general, $\mathrm{N}_{\mathrm{i}}$ is defined as a square neighbourhood window around voxel $i$ with a user-defined "radius" $R_{\text {sim. }}$. The similarity function $w\left(N_{p}, N_{q}\right)$ is calculated as:

$$
w\left(N_{p}, N_{q}\right)=\frac{1}{Z_{p}} e^{--\frac{d\left(N_{p}, N_{q}\right)}{h^{2}}}
$$

$Z_{p}$ is the normalizing constant, $h$ is an exponential decay control parameter and $d$ is a Gaussian weighted squared Euclidian distance of all the voxels of each neighbourhood:

$$
d\left(N_{p}, N_{q}\right)=\left\|G(\rho)_{p-q}\left(N_{p}-N_{q}\right)\right\|_{R s i m}^{2}
$$

$G(\rho)$ is a normalized Gaussian weighting function with zero mean and $\rho$ standard deviation (usually set to 1 ) that penalizes voxels far from the center of the neighbourhood window giving more weight to voxels near the center of the window in the distance computation.

Figure 1 represents the voxel in restoration, characterised in the comparisons by its neighbourhood, $\mathrm{N}_{\mathrm{p}}$, and the different neighbourhoods that are compared to the one being restored, $\mathrm{N}_{\mathrm{q}}$.

In Equation (8) there is a special case when $q=p$. As the distance from a voxel to itself is zero, it can produce an over-weighting effect. To solve this problem in the original NL-Means method, $\mathrm{d}\left(N_{p}, N_{p}\right)$ is calculated as:

$$
\mathrm{d}\left(\mathrm{N}_{\mathrm{p}}, \mathrm{N}_{\mathrm{p}}\right)=\min _{\forall p \neq q}\left(\mathrm{~d}\left(\mathrm{~N}_{\mathrm{p}}, \mathrm{N}_{\mathrm{q}}\right)\right)
$$




\section{Method optimizations}

A number of optimizations can be used that increase the accuracy of the results of the method and that specially reduce the computational time. Two optimizations were introduced in the study and they are presented below.

\section{1) Management of singular points}

In equation (9) the distance assigned to the voxel being processed to itself is set to the minimum of the other distances in the search window. Equation (9) avoids overweighting effects and allows more noise removal. However, that correction has the disadvantage of blurring singular points (i.e. voxels with no similar patches, such as image corners and peaks or valleys) by averaging them with voxels from non-similar patches. To overcome this situation, a modification to equation (9) is proposed: The distance from the voxel to itself is only calculated by means of the surrounding voxels if the distance is above a fixed threshold. We experimentally fixed this value to $4 \sigma^{2}$ (with $\sigma$ the estimated noise standard deviation) which corresponds to the maximum distance that a similar voxel can have.

$$
\mathrm{d}\left(\mathrm{N}_{\mathrm{p}}, \mathrm{N}_{\mathrm{p}}\right)=\min \left(4 \sigma^{2}, \min _{\forall q \neq p}\left(\mathrm{~d}\left(\mathrm{~N}_{\mathrm{p}}, \mathrm{N}_{\mathrm{q}}\right)\right)\right)
$$

2) Voxel pre-selection

Another useful improvement is to perform a voxel pre-selection in order to avoid useless computations and to improve filtering results by excluding non similar voxels in the averaging process (Mahmoudi, Sapiro, 2005; Coupe et al, 2007).

In the present work, the speedup was implemented by means of selecting those voxels that their first local moment $\mu$ (mean value of a $3 \times 3 \times 3$ volume patch) was not located farther away than $k \sigma / \sqrt{n}$ ( $n$ being the number of pixels used to compute the mean, 27 in this case). 


$$
w\left(N_{p}, N_{q}\right)=\left\{\begin{array}{l}
\frac{1}{Z_{p}} e^{-\frac{d\left(N_{p}, N_{q}\right)}{h_{i}^{2}}} \quad \text { if }\left(\left|\mu_{p}-\mu_{q}\right|<\mathrm{k} \sigma / \sqrt{\mathrm{n}}\right) \\
0 \quad \text { otherwise }
\end{array}\right.
$$

A value of $k=3$ was used to set the pre-selection threshold, which corresponds to the third quartile of a standard normal distribution. Mean value differences higher than this threshold have a very low probability to be similar to the voxel being filtered and its context. We did not include second order moments in the voxel pre-selection process in contrast with what Coupé et al. (2007) proposed, as we found that it was a too restrictive selection criterion.

\section{Implementation}

The adaptive bilateral filter was implemented with a Gaussian edgestopping function. The NL-Means filter was implemented in 3D. One dimension corresponded to time while the other two dimensions corresponded to the slice data (i.e. image plane plus time) to achieve reasonable processing time; but a 4D implementation (i.e. 3D volume plus time) could also be used to increase the quality of the filtering at the expense of increasing the computational burden and thus the processing time. In this study, all the filtering methods were applied after movement correction. The isotropic 3D implementation of the filter processed one fMRI slice at a time and all the temporal positions for that slice. In each comparison, only windows around the selected voxel were used (as a result, time is used in the filtering, but only nearby temporal points are included, so the method can be used to filter blocked and event-related design fMRI data).

\section{Parameter settings}

Optimum parameters were selected for each filtering method. That is, for Gaussian kernel filtering, FWHM was set to 3 voxels, an extent similar to that in the shapes in the activation mask ( $\sigma$ was 1.274 voxels); $S$ was $7 \times 7$. For the Adaptive 
Bilateral Filtering, $\sigma$ and $S$ were the same as in the Gaussian filter, while $\theta$ was calculated directly from the data as the local standard deviation of a squared window of size $3 \times 3$ surrounding the current pixel; for NL-Means, after an exhaustive search of possible parameters and tuning to maximize the quality of the results over usual noise levels in fMRI data, Rsim was 1 (size of the similarity

Neighbourhood $3 \times 3 \times 3$ ), $\mathrm{S}$ was $7 \times 7 \times 7$ and $\mathrm{h}=\sqrt{2} \sigma_{n}$, with $\sigma_{n}$ the standard deviation of noise.

\section{Synthetic data}

To evaluate the different filtering schemes, a software phantom was generated with activation structures similar in shape to the ones present in Friman et al. (2003). The structures for the phantom were encoded in a mask of activation. Shapes in the mask had different intensity values, with higher intensity in the center of each shape and a decreasing intensity toward the bounds of the shape. Structures consisted of 3D boxes, spheres and arrows. One volume was generated as the mean of three real fMRI volumes, obtained while a healthy subject was at rest. The volume was replicated 99 times, thus obtaining 100 identical volumes, representing the fMRI data.

An ideal hemodynamic response function (hrf) (5\% amplitude) was introduced. The hrf was created with a TR $=2$ seconds and a block size of 20 seconds. Two conditions were encoded in the hrf: Activation and Rest. The hrf was replicated five times. Thus, it consisted of 100 temporal positions. The fMRI data volumes were convolved with the hrf only in the spatial voxels that were included in the mask of activation.

Afterwards, the volumes were convolved with simulated trends and drifts from the scanner, typically observed in fMRI. These were introduced by convolving the volumes with a smooth third-order polynomial that simulated slow local changes in the MR signal. 
As the magnitude of the MRI signal is the square root of the sum of the squares of Gaussian distributed real and imaginary parts, the acquired fMRI data follow a Rician distribution (Sijbers, Dekker, 2004). Therefore, Rician noise was finally added including $1 \%, 3 \%, 5 \%, 7 \%$ and $10 \%$ levels of the mean intensity of the fMRI volumes. Noise was introduced by adding Gaussian noise to both real and imaginary parts and then computing the magnitude volume image.

The different filtering methods were compared using well-known quantitative measures. The first measure was Root Mean Square Error (RMSE) that compared the original (before Rician noise added) signal to the filtered signal. A second measure was the functional Signal-to-Noise Ratio (fSNR) at each activated voxel. For extracting a measure of the fSNR, a Statistical Parametric Map was generated for each filtering technique and each noise level. Then the fSNR was calculated as the estimation of the contrast divided by its noise estimation, in a voxel-wise basis, where only activated voxels were taken into account. The fSNR was at last summarized as the mean of the fSNR value of all activated voxels.

Finally, false positive and true positive rates for the detection of activated voxels, and therefore sensitivity and specificity, were assessed for the different noise levels.

\section{Clinical data}

As a corpus of real clinical data for the comparison of the methods, experimental fMRI data was obtained from a study that analyzed auditory emotional response in schizophrenia. fMRI volumetric images were acquired every two seconds in a block design, where two blocks of 20 seconds were sequentially presented: one rest block (no sound) and one block consisting of 13 high emotional words selected from those that a schizophrenic cohort of patients usually heard. fMRI was acquired in both schizophrenic patients $(n=11)$ and healthy subjects $(n=10)$. The study is described in detail in Sanjuán et al. 2007. 
Images were performed applying a dynamic Echo Planar Imaging T2* weighted sequence (TR=2000 ms, TE=50 ms, $5 \mathrm{~mm}$ slice thickness with no interslice gap, 24 slices, acquisition matrix $=96 \times 128$, field of view $=220 \mathrm{~mm}$ and flip angle $=65^{\circ}$ ). The voxel size was $3.27 \mathrm{~mm} \times 1.72 \mathrm{~mm} \times 5 \mathrm{~mm}$.

To compare the described filtering methods, the same process was performed to correct the fMRI images and to statistically analyse them, except for the noise filtering step. Movement correction was applied by means of SPM5 (Wellcome Trust Centre for Neuroimaging, London, UK) realignment procedure the fMRI data. Then the fMRI images were noise filtered by either by NL-Means, Gaussian Filter of Adaptive Bilateral Filter. They were finally normalized to the MNI template (Montreal Neurological Institute, Montreal, Canada) with the same normalization parameters as in Sanjuán et al. 2007.

The Gaussian Filter was applied with FWHM $=6 \times 6 \times 6 \mathrm{~mm}$. The Adaptive Bilateral filter was applied with $\mathrm{S}=7 \times 7 \times 7$ and FWHM $=6 \times 6 \times 6 \mathrm{~mm}$. Finally the NL-Means filter was applied with parameters: $S=7 \times 7 \times 7, R_{\text {sim }}=1 \times 1 \times 1$ and $h$ $=\sqrt{2} \sigma_{n}$, where $\sigma_{n}$ was the estimated standard deviation of noise.

Noise was estimated from the standard deviation of the difference (SDD) of 2 consecutive fMRI volume images at regions with non-null signal where the noise distribution could be approximated by a Gaussian distribution:

$$
\sigma_{n}=\mathrm{SDD} / \sqrt{2}
$$

Clinical validation of the methods was performed visually and by inspection of the temporal curves in voxels estimated as highly activated (after the generation of a parametric map for each filtering method). There is no ground truth as to what voxels are active, when real fMRI data is considered. Thus, visual inspection was used to detect differences in the cluster sizes and the functional patterns in voxels that survived a $p<0.05$, Bonferroni corrected. 


\section{EXPERIMENTS AND RESULTS}

Results were assessed for both synthetic and clinical data and are presented in the following subsections.

\section{Synthetic data}

Firstly, the original and the optimized versions of the NL-Means filter were evaluated. In figure 2 RMSE results for both versions are shown. The optimized version improved the accuracy of the classic method while requiring significant less time to process the data (a speed factor of approximately two was achieved in the optimized version of the filter). The subsequent results in this study regarding the NL-Means method are referred to its optimized version.

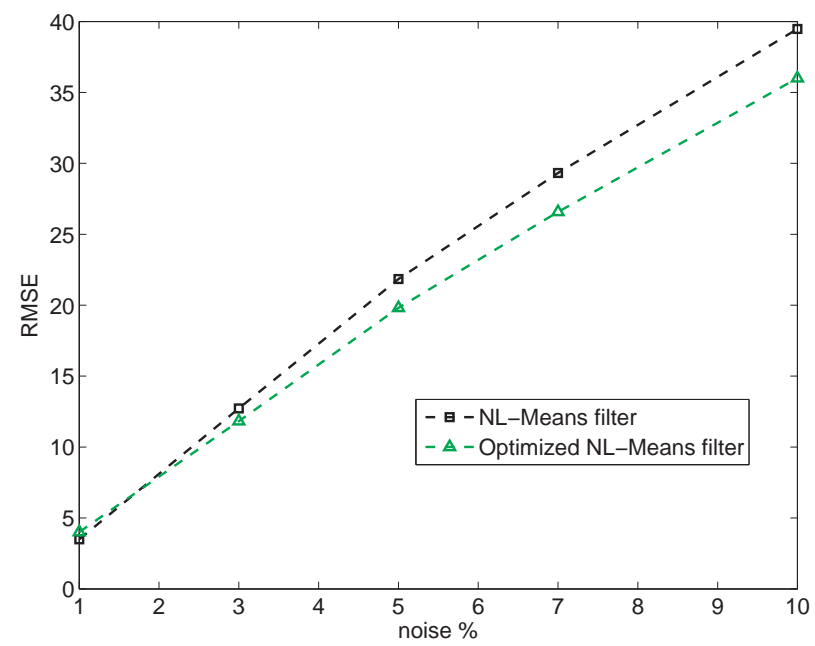

Figure 2. RMSE corresponding to the original NL-Means filter applied to synthetic data and RMSE corresponding to the Optimized NL-Means filter.

RMSE is shown for each compared filtering method and noise level in Figure 3. When the noise present in the fMRI data was low (e.g. 1\%) Gaussian smoothing and Adaptive Bilateral filtering methods showed a higher RMSE value than in the unfiltered data, due to their spatial blurring of the fMRI data. However, when noise was higher (i.e. above 3\% for Adaptive Bilateral and 6\% for Gaussian), 
the filtering methods showed lower RMSE values than the RMSE for the unfiltered data. In the case of the NL-Means filter, both original and optimized versions produced a lower RMSE than that in the unfiltered images for all noise levels. Finally, normalized fSNR (summarized fSNR of the filtered signal divided by the summarized fSNR of the original signal) was higher for the NL-Means filter compared to the other filters (Figure 4).

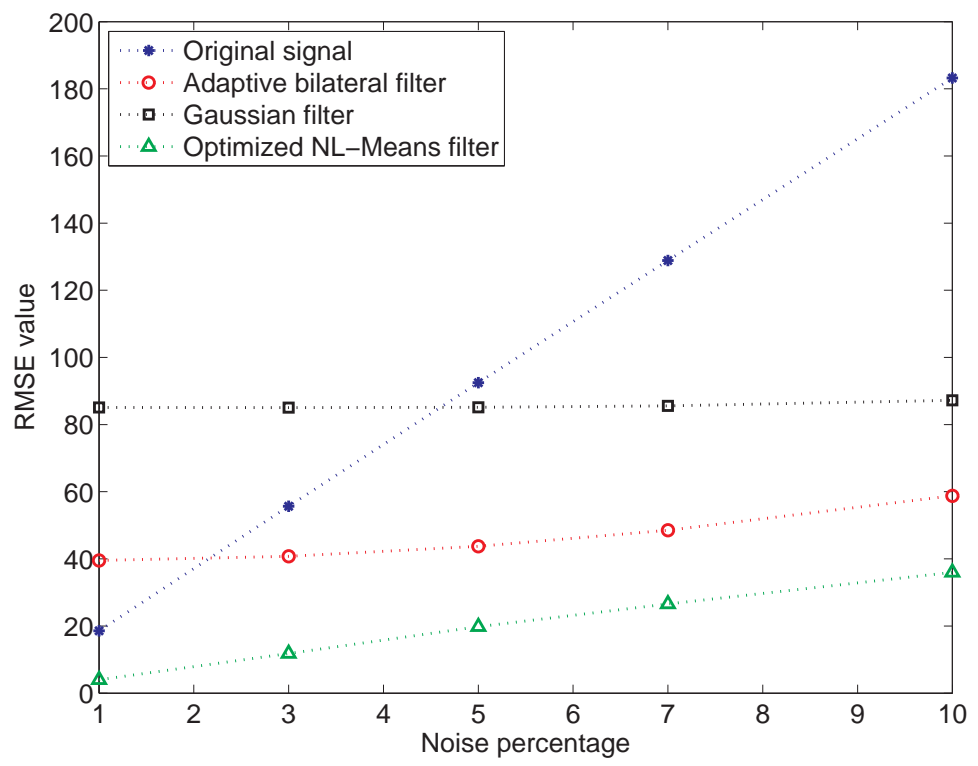

Figure 3. RMSE value comparing the original (without noise) and the filtered fMRI volumes. RMSE value for NL-means filter was lower for all noise levels. 


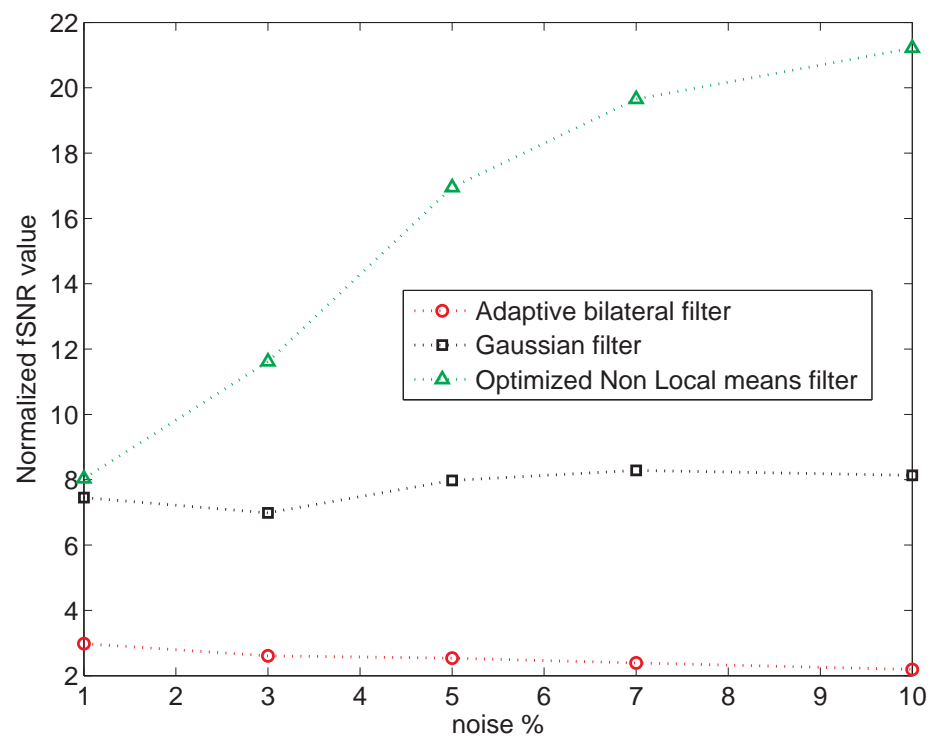

Figure 4. Normalized fSNR for the three compared filtering methods. Notice that NL-Means filtered normalized fSNR is higher for all the tested noise levels.

The computational time was around 4 minutes for the complete data set when the Gaussian filter was applied, while it was approximately one hour and 20 minutes with the Adaptive Bilateral filter and 5 minutes with the optimized NLMeans filter (computing times obtained on a $2.4 \mathrm{GHz}$ Pentium 4 with 2 GB RAM using MATLAB 2006b, running Windows 2000).

Sensitivity and specificity in the detection of activated voxels were also assessed for the three filtering methods. Figure 5 visually demonstrates the recovery properties of the three methods, for a $3 \%$ noise (activation maps obtained with a multiple comparison Bonferroni correction, $p<0.05$ ). The NL-Means filter allowed the detection of the dotted line shape of activation (left-most axial slice), the Adaptive Bilateral Filter and the unfiltered data let the detection of some of the voxels and finally no voxel in the dotted line shape was detected when the Gaussian Filter had been applied. The recovery by the three methods of other shapes: spheres, cubes and lines is also shown in Figure 5, demonstrating the highest resemblance between the activation mask and the activation corresponding to the NL-Means filtered data. 


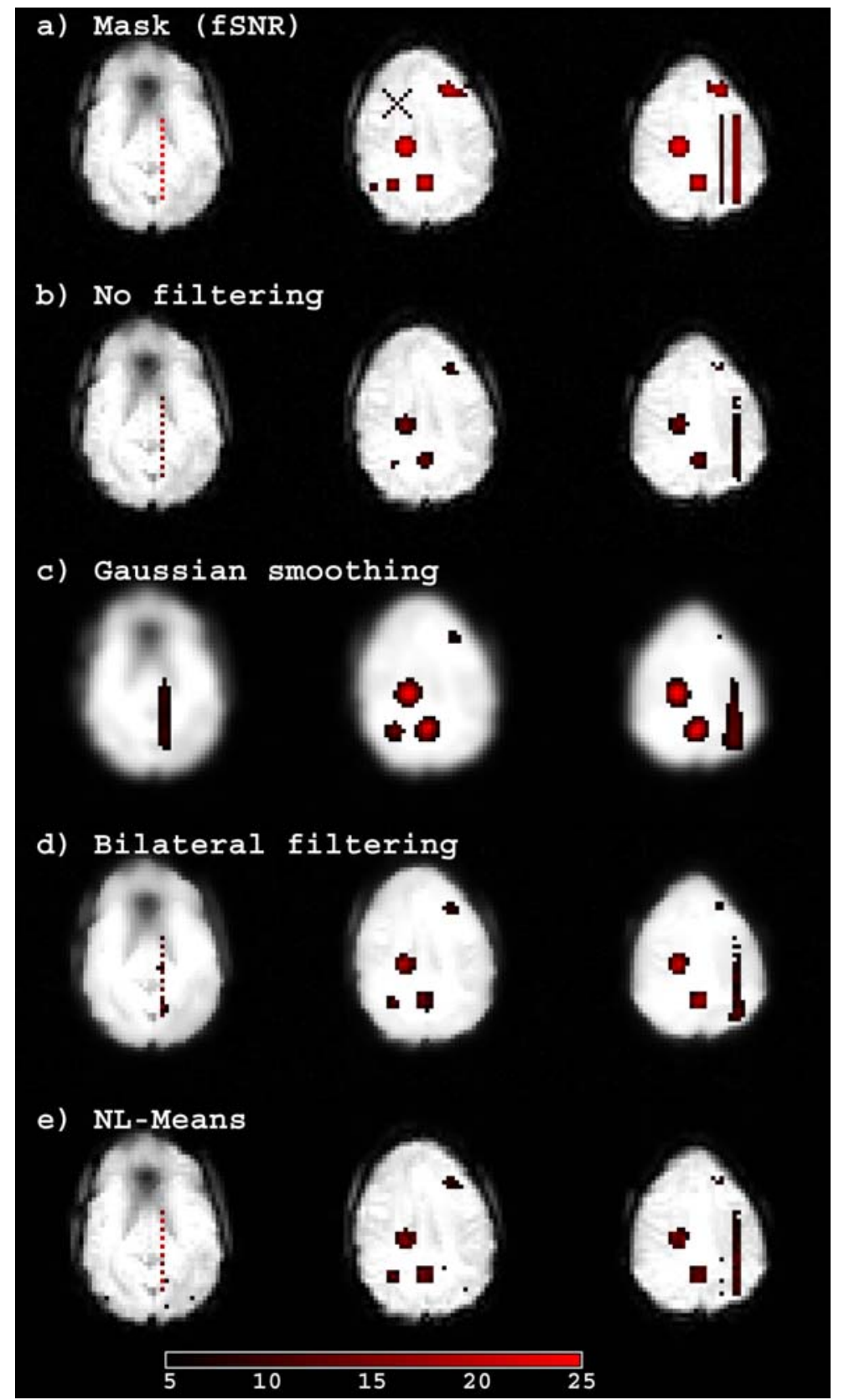

Figure 5. Visual comparison of the different filtering methods, noise $3 \%$, fMRI signal $5 \%$. a) Pattern of activation introduced (similar to the masks in Friman et al, 2003), shown in three axial slices (notice spatial gradient of activation). b)-e) Detected activation in the same three slices, with colour-coded maps: b) Activation detection without any filtering applied to the noisy images. c-e) activation detection after applying, respectively, Gaussian, Bilateral and NL-Means filtering methods. Colour gradient indicates t-value, as shown in the bottom colour-bar. 
Figure 6 shows the Sensitivity and Specificity characteristics in the detection of activated voxels, represented by their ROC curves, for a $1 \%$ noise level, for each filtering method. The original signal had an elevated ratio of true positives against false positives. Few voxels are typically detected in the original signal but these are usually true positives. However the filtering methods introduce not only true but false positives when restoring the data. Gaussian filtering obtained the lowest area under the ROC curve, because the method created false positives around the areas of activation. Adaptive Bilateral filtering had a higher area under the ROC curve, due to its respectful treatment of edges. Finally, NLMeans had the highest area under the ROC curve, with curve that was very similar to the one in the original data.

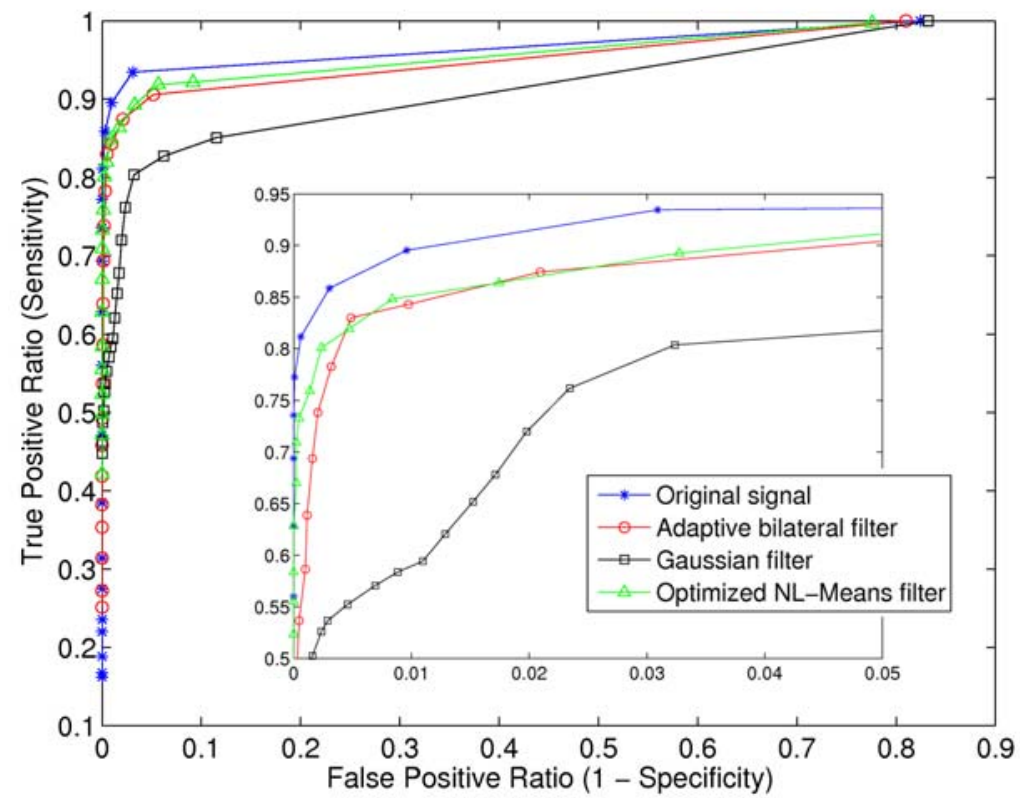

Figure 6. ROC curves, showing the sensitivity and specificity in the detection of activated voxel, for the different filtering methods and the original image without filtering (noise $=1 \%$; signal $=5 \%$ ). A close-up in the region of high specificity is also shown.

\section{Clinical data}

Four maps from a single subject were obtained by means of SPM5, one for each filtering technique and one for the unfiltered data, with an estimation of signal by means of a General Linear Model. Serial correlations were taken into account 
by means of an autoregressive model (Purdon and Weisskoff, 1998). A region was selected that was only detected by NL-Means in the Superior Temporal Gyrus, a key gyrus in the study of schizophrenia. With a Bonferroni threshold corresponding to a $p<0.05$ ( $T>5.48)$, no voxel was detected as activated in the region around (38,$26,20)$ when data had been Gaussian filtered. No voxels were detected as activated in the original data without filtering, nor after Adaptive Bilateral Filter was applied. Finally, 23 connected voxels around $(38,-26,20)$ were statistically detected with the same Bonferroni threshold when NL-Means filtering was applied.

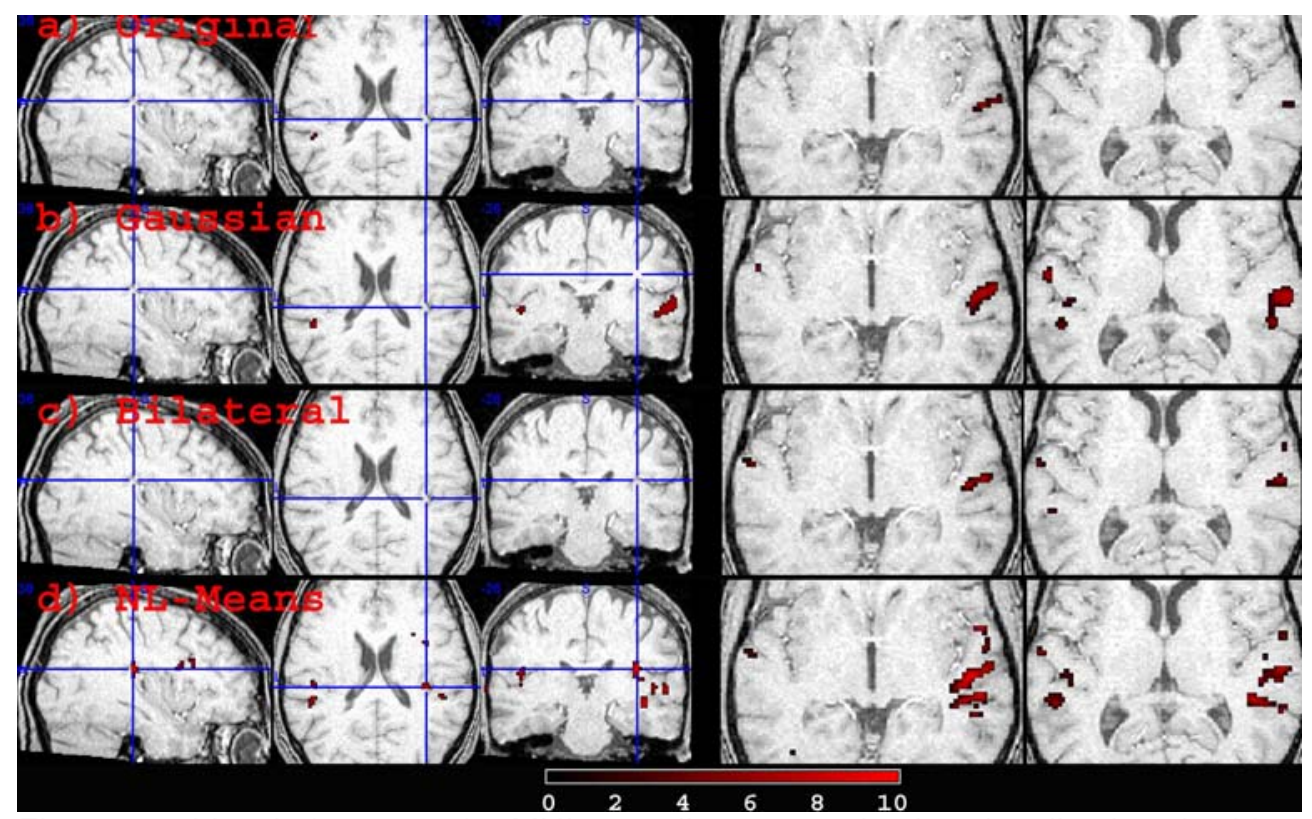

Figure 7. Voxel $(38,-26,20)$-MNI coordinates- activation localization in blue crosshairs, over the subject's anatomical saggital, axial and coronal images for (a) the original unfiltered fMRI data, (b) the Gaussian filtered data, (c) the Adaptive Bilateral filtered data and (d) NL-Means filtered data, respectively. Two more axial slices show the ability of restoring the fMRI data by the shapes and statistical power (color-coded). Notice how NL-Means activations have higher statistical values and show shapes that follow the sulcal anatomy. Colour gradient in a)-d) indicates t-value, as shown in the bottom colour-bar. Coordinate $(38,-26,20)$ was selected here as it represented a cluster that was only detected after applying the NL-Means filter.

The temporal signal in the voxel with coordinates $(38,-26,20)$ was also observed (Figure 8). The ideal hemodynamic signal for the voxel was plot along with the data after each filtering method or unfiltered. As it can be appreciated, the signal restored with the NL-Means method was similar to the ideal expected hrf in 
the specific voxel, while it was not so well appreciated in the other filtering methods.

a) Original unfiltered signal, $\mathrm{T}=4.97$

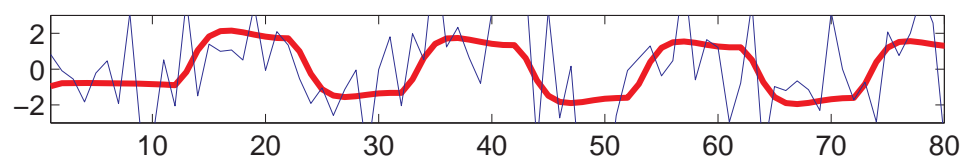

b) Gaussian filtered signal, $T=3.37$

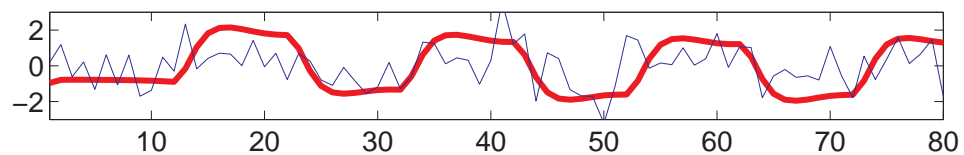

c) Adaptive Bilateral filtered signal, $T=3.32$

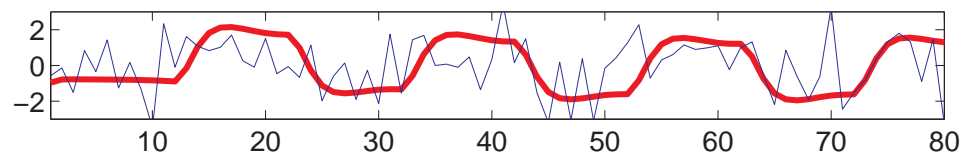

d) Optimized NL-Means filtered signal, $T=9.75$

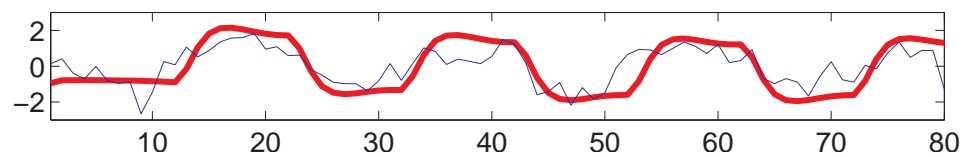

Figure 8. Restored and unfiltered fMRI signal in the voxel with MNI coordinates $(40,-26,20)$. Haemodynamic filtered signal (blue) and ideal response (magenta, thick line) for (a) the original unfiltered fMRI, (b) the Gaussian kernel filtered, (c) the Adaptive Bilateral filtered and (d) the NL-Means filtered fMRI data, respectively, are shown. T value corresponding to the mentioned voxel is also shown for each method. 


\section{DISCUSSION}

According to our results and to some recent studies (Coupé et al, 2007; Manjon et al, 2008; Wiest-Daesslé et al, 2007), NL-Means has shown to be an excellent filter for MRI denoising. Filtering with NL-Means provided enhanced activation localization without mixing intensities in the edges of structures nor in the edges of functional patterns, especially preserving brain activation in areas of bordering sharp transitions (see Figure 5). To the best of our knowledge, this is the first time that the NL-Means filter has been proposed for fMRI data restoration.

While the Gaussian Filter has been the selected choice in many fMRI research and clinical studies because it enhances fSNR at the expense of very low computational cost, it also generates false positives outside of the brain and in the interfaces between tissues. The Adaptive Bilateral method, recently adapted to fMRI filtering, is more respectful with the data than the Gaussian kernel method. However, it is effectively non-linear, and it does not recover the fMRI signal near the edges of anatomical structures. Besides, its performance decreases rapidly as the noise levels increases due to the low robustness of its similarity function (point based instead of region based). Other filtering methods introduce complex frameworks that make the data and the noise interact in a way that is not exactly understood.

NL-means filtering denoises the $\mathrm{fMRI}$ data before the statistical decision process, respecting the structure of the noise. Thus, it is a different approach to that of modelling the noise inside the statistical decision-on-activation process. Advantages and drawbacks can be attributed to both approaches. The main advantage that statistical model-independent filtering methods have is that different statistical and exploratory techniques can be applied on the data after filtering.

Some drawbacks to the NL-Means filter should be finally mentioned. Firstly, as the method does not transform the data, not-normally or coloured-noise distributed noise will remain coloured. Therefore, the Random Field Theory (RFT) could not be applied to images filtered by NL-Means. RFT lets us define a 
threshold less conservative than Bonferroni. The validity of this thresholding method, under normal data distribution assumptions, is assured (Worsely, 1992; Worsley, 1996). However, we believe that the application of non parametric statistical methods is the natural choice when feasible. Other fMRI activation statistical or exploratory procedures could also be explored with fMRI data that had been filtered by NL-means, such as the ones based on advanced spatial normalization: Freesurfer (Athinoula A. Martinos Center, Massachusetts General Hospital, Massachusetts, USA), Caret (Van Essen Lab, Washington University in St. Louis, St. Louis, USA), etc.

Secondly, another known drawback to the method is the computational burden associated to this filter. There have been approaches to reduce the time used by NL-Means, such as Coupé et al, 2007, by blockwise processing and multithreading / multicore approaches. Time could also be highly reduced by the use of a Graphics Processing Unit (GPU) or GRID (Blanquer et al, 2006) technologies, due to the possibility of decomposing the data filtering and due to the parallel processing characteristics of the GPUs and GRID.

In contrast with the two mentioned drawbacks, fSNR is improved by NLMeans fMRI filtering while sensitivity and specificity in detecting activated voxels are enhanced by means of this filtering method. These two properties make the NL-Means filter interesting for clinical fMRI data processing, as it has the ability to detect fMRI signal in "difficult" areas (see Figure 7). Areas of activation in the interface between gray matter, white matter and / or CSF could pass undetected with other filtering methods. We found an activation area that could not be detected with the rest of the filtering methods. Areas of activation near tumours and malformations will more likely be detected with NL-Means too, as they also represent an interface between tissues. 


\section{Conclusion}

We have demonstrated the ability of NL-means filter to reduce the noise and enhance the localization of the activation areas. It has also been shown that a better specificity and sensitivity in the localization of activated voxels is obtained with the application of the NL-Means filter. These properties make the filter a directly applicable tool for fMRI pre-processing.

The main future optimization for fMRI of the NL-Means method should be the $4 \mathrm{D}$ implementation of the algorithm; this will require the application of parallel techniques and the use of technologies such as GPU processing (Novotny et al, 2007).

\section{ACKNOWLEDGEMENTS}

Authors would like to thank Dr. Peset University Hospital and Quirón Valencia Hospital for supplying the real MR images used in this work. We would also like to express gratitude to the Conselleria de Empresa, Universidad y Ciencia, Valencia for their financial support (GV/2007/99 project). This research was also supported by the Asociación para el Desarrollo y la Investigación en Resonancia Magnética (ADIRM) Foundation. We would like to thank the R\&D+i Linguistic Assistance Office at the Universidad Politécnica de Valencia for their help in revising and correcting this paper. 


\section{REFERENCES}

Bandettini P. Functional MRI today. Int J Psychophysiol, 63 (2007), pp. 138-145.

Blanquer I, Hernández V, Monleón D, Carbonell J, Moratal D, Celda B, Robles M, Martí-Bonmatí. Using the grid to analyze the pharmacokinetic modelling after contrast administration in dynamic MRI. Stud Health Technol Inform. (2006), pp. 12082-92.

Brox T, Cremers D. Iterated Nonlocal Means for Texture Restoration In Scale Space and Variational Methods in Computer Vision (2007), pp. 13-24.

Buades A, Coll B, Morel JM. A review of image denoising algorithms, with a new one. Multiscale Modeling and Simulation (SIAM interdisciplinary journal), 4 (2005), pp. $490-530$.

Buades A, Coll B, Morel JM. Nonlocal Image and Movie Denoising. International Journal of Computer Vision, 76 (2008), pp. 123-139.

Coupé P, Yger P, Prima S, Hellier P, Kervrann C, Barillot C. An Optimized Blockwise Non Local Means Denoising Filter for 3D Magnetic Resonance Images. Transactions on Medical Imaging (2007): In press.

Desco M, Hernández JA, Santos A, Brammer M. Multiresolution Analysis in fMRI: Sensitivity and Specificity in the Detection of Brain Activation. Human Brain Mapping 14 (2001), pp. 16-27.

Descombes $X$, Kruggel F, von Cramon DY. fMRI signal restoration using a spatiotemporal Markov Random Field preserving transitions. Neuroimage 8 (1998), pp. 340-9.

Durand F, Dorsey J. Fast bilateral filtering for the display of high-dynamic-range images. SIGGRAPH (2002) pp. 257-266.

Flandin G Penny WD. Bayesian fMRI data analysis with sparse spatial basis function priors. Neuroimage 34 (2007) pp. 1108-25.

Friman $\mathrm{O}$, Borga $\mathrm{M}$, Lundberg $\mathrm{P}$, Knutsson $\mathrm{H}$. Adaptive analysis of $\mathrm{fMRI}$ data. Neuroimage. 19 (2003), pp. 837-45.

Friman O, Carlsson J, Lundberg P, Borga M, Knutsson $\mathrm{H}$. Detection of neural activity in functional MRI using canonical correlation analysis. Magn Reson Med 45 (2001), pp. 323-330.

Friston KJ, Josephs O, Zarahn E, Holmes AP, Rouquette S, Poline J. To smooth or not to smooth? Bias and efficiency in fMRI time-series analysis. Neuroimage. 12 (2000), pp. 196-208. 
Geerts J, Martens M, Vandevenne JE, Gelin G, Grieten M, Weyns F, Stinissen P, Palmers $Y$, Wuyts J. Functional magnetic resonance imaging for preoperative localisation of eloquent brain areas relative to brain tumours: clinical implementation in a regional hospital. JBR-BTR 90 (2007), pp. 258-63.

Gössl C, Auer DP, Fahrmeir L. Bayesian spatiotemporal inference in functional magnetic resonance imaging. Biometrics 57 (2001) pp. 554-62.

Kim HY, Giacomantone J, Cho ZH. Robust anisotropic diffusion to produce enhanced statistical parametric map from noisy fMRI. Computer Vision and Image Understanding 99 (2005) pp. 435-452.

Mahmoudi M, Sapiro G. Fast image and video denoising via nonlocal means of similar neighborhoods. Signal Processing Letters, IEEE 12 (2005), pp. 839-842.

Majos A, Tybor K, Stefańczyk L, Góraj B. Cortical mapping by functional magnetic resonance imaging in patients with brain tumors. European Radiology 15 (2005), pp. 1148-1158.

Manjon JV, Carbonell-Caballero J, Lull JJ, Garcia-Marti G, Marti-Bonmati L, Robles M. MRI denoising using Non-Local Means. Medical Image Analysis: In Press (2008).

McKeown M, Makeig S, Brown G, Jung T, Kindermann S, Bell A, Sejnowski T. Analysis of $\mathrm{fMRI}$ data by blind separation into independent spatial components. Hum Brain Mapp 6 (1998), pp. 160-188.

Novotny PM, Stoll JA, Vasilyev NV, del Nido PJ, Dupont PE, Zickler TE, Howe RE. GPU based real-time instrument tracking with three-dimensional ultrasound. Medical Image Analysis. 11 (2007), pp. 458-464.

Penny WD, Trujillo-Barreto NJ, Friston KJ. Bayesian fMRI time series analysis with spatial priors. Neurolmage 24 (2005) pp. 350-362.

Petersson KM, Nichols TE, Poline JB, Holmes AP. Statistical limitations in functional neuroimaging. II. Signal detection and statistical inference. Philos Trans R Soc Lond B Biol Sci. 354 (1999), pp. 1261-81.

Petrella JR, Shah LM, Harris KM, Friedman AH, George TM, Sampson JH, Pekala JS, Voyvodic JT. Preoperative Functional MR Imaging Localization of Language and Motor Areas: Effect on Therapeutic Decision Making in Patients with Potentially Resectable Brain Tumors. Radiology 240 (2006), pp. 793-802.

Pujol J, Deus J, Acebes JJ, Villanueva A, Aparicio A, Soriano-Mas C, Ortiz H, Conesa $G$. Identification of the sensorimotor cortex with functional MRI: frequency and actual contribution in a neurosurgical context. J Neuroimaging 18 (2008), pp. 28-33. 
Purdon PL, Weisskoff RM. Effect of temporal autocorrelation due to physiological noise and stimulus paradigm on voxel-level false-positive rates in fMRI. Hum Brain Mapp 6 (1998) , pp. 239-49.

Reimold M, Slifstein M, Heinz A, Mueller-Schauenburg W, Bares R. Effect of spatial smoothing on t-maps: arguments for going back from t-maps to masked contrast images. J Cereb Blood Flow Metab. 26 (2006), pp. 751-9.

Ruff I, Petrovich Brennan N, Peck K, Hou B, Tabar V, Brennan C, Holodny A. 2008. Assessment of the Language Laterality Index in Patients with Brain Tumor Using Functional MR Imaging: Effects of Thresholding, Task Selection, and Prior Surgery. AJNR Am J Neuroradiol 29 (2008), pp. 528-535.

Sanjuán, J., Lull, J.J., Aguilar, E.J., Marti-Bonmati, L., Moratal, D., Gonzalez, J.C., Robles, M., Keshavan, M.S. Emotional words induce enhanced brain activity in schizophrenic patients with auditory hallucinations. Psychiatry Res 154 (2007), pp. 21-29.

Smith SM and Brady JM. SUSAN - a new approach to low level image processing. Int. Journal of Computer Vision, 23 (1997), pp.45-78.

Sole A, Shing-Chung Ngan, Sapiro G, Xiaoping Hu, Lopez A. Anisotropic 2-D and 3-D averaging of fMRI signals. Medical Imaging, IEEE Transactions on 20 (2001), pp. 86-93.

Tomasi C and Manduchi R. Bilateral Filtering for gray and color images. Sixth International Conference on Computer Vision (1998), pp. 839-46.

Vlieger E, Majoie CB, Leenstra S, den Heeten GJ. Functional magnetic resonance imaging for neurosurgical planning in neurooncology. European Radiology 14 (2004), pp. 1143-1153.

Walker SA, Miller D, Tanabe J. Bilateral spatial filtering: refining methods for localizing brain activation in the presence of parenchymal abnormalities.

Neuroimage. 33 (2006), pp. 564-9.

Wiest-Daesslé N, Prima S, Coupé P, Morrissey SP, Barillot C. Non-local means variants for denoising of diffusion-weighted and diffusion tensor MRI. Lecture Notes in Computer Science 10 (2007), pp. 344-351.

Wink AM, Roerdink JB. Denoising functional MR images: a comparison of wavelet denoising and Gaussian smoothing. IEEE Trans Med Imaging. 23 (2004), pp. 37487.

Yaroslavsky LP. Digital Picture Processing - An Introduction. Springer Verlag (1985). 
Woolrich M, Jenkinson M, Brady J, Smith S. Fully Bayesian spatio-temporal modeling of FMRI data. Medical Imaging, IEEE Transactions on 23 (2004) pp. 213231. 


\section{6.b. - Addendum}

As the manuscript is the main part of this chapter, some errors that have been found are transcripted here. The manuscript was not modified, as it is the one that has been already published. Changes are presented in blue colour. Pages are referred here in the thesis document, as the paper is still in the revision stage.

p.83 Introduction. $\mathrm{fMRI}$ as an aid to tumour resection is introduced but the focus of the paper is schizophrenia. This was not clearly explained in the manuscript. This will be changed in the final manuscript, which will start with:

The application of the fMRI technique is becoming wider everyday. Its ability to detect changes in function in the healthy and unhealthy brain makes this non-invasive technique suitable to study brain activity in-vivo. Even though this paper centres on the application of NL-Means to a better localization of activation areas in schizophrenia fMRI, the application of fMRI as a clinical test of functional localization in tumour resection clearly shows the advances of fMRI during the last years. The developed method could also clearly aid in these cases.

p.86 Equation (3) should read:

$$
G(\sigma)_{p-q}=\frac{1}{\sqrt{2 \cdot \pi \cdot \sigma^{2}}} e^{-\frac{(p-q)^{2}}{2 \sigma^{2}}}
$$


p.88 Equation (8) should read:

$$
d\left(N_{p}, N_{q}\right)=\left\|G(\rho)\left(N_{p}-N_{q}\right)\right\|_{R s i m}^{2}
$$

Before it read: $\mathbf{G}(\rho)_{p-q}$ instead of $\mathbf{G}(\rho)$. The Gaussian kernel $\mathbf{G}(\rho)$ is a fixed kernel that gives more weight to central voxels in the neighbourhoods being compared than the weight given to voxels away of the center of the neighbourhoods.

p.90. Parameter settings. $\sigma$ could have been stated in units of $\mathrm{mm}$ as voxels are anisotropic:

Optimum parameters were selected for each filtering method. That is, for Gaussian kernel filtering, FWHM was set to 3 voxels in each axis, an extent similar to that in the shapes in the activation mask ( $\sigma$ was 1.274 voxels in each axis, i.e. $4.16598 \times 2.19128 \mathrm{~mm}$ );

p.91 Parameters are restated in $\mathrm{mm}$ units:

The Gaussian Filter was applied with FWHM $=6 \times 6 \times 6 \mathrm{~mm}$. The Adaptive Bilateral filter was applied with $S=7 \times 7 \times 7$ voxels, i.e. $22.89 \times 12.04 \times 35 \mathrm{~mm}$, and FWHM $=6 \times 6 \times 6 \mathrm{~mm}$.

Finally the NL-Means filter was applied with parameters: $\mathrm{S}=$ $7 \times 7 \times 7$ voxels $(22.89 \mathrm{~mm} \times 12.04 \mathrm{~mm} \times 14 \mathrm{~s})$, Rsim $=1 \times 1 \times 1$ voxels $(3.27 \mathrm{~mm} \times 1.72 \mathrm{~mm} \times 2 \mathrm{~s})$ and $\mathrm{h}=\sqrt{2} \sigma_{n}$, where $\sigma_{n}$ was the estimated standard deviation of noise. 


\section{6.c. - Supplementary results}

NL-Means has shown to be a powerful, while respectful, filtering method. Clinical tests that have been conducted with fMRI data from the schizophrenia research project with the NL-Means method were presented in the manuscript. In addition to those results, spatial smoothing filtered and NL-Means filtered data is presented here for two schizophrenic patients and for a healthy subject. Preliminary results show that localization of activation to emotional semantic auditory stimulation is closer to gyral anatomy in the case of NL-Means filtering, supporting the results presented in the manuscript. By the other side, results lead us to the conclusion that the specific localization of activation areas after the application of NL-Means will pose inter-subject analysis challenges. Activation clusters follow better the shapes of the gyri with respect to spatial smoothing, while maintaining the detectability of the signal. Those results are shown bellow, in Figures 22 to $\underline{24}$, in addition to the clinical data results shown in the submitted manuscript. The axial slices in Figure 22 and Figure 23 demonstrate a better localization power in NL-Means, specially shown by the shapes of the clusters of activation that follow naturally the sulcal anatomy. 


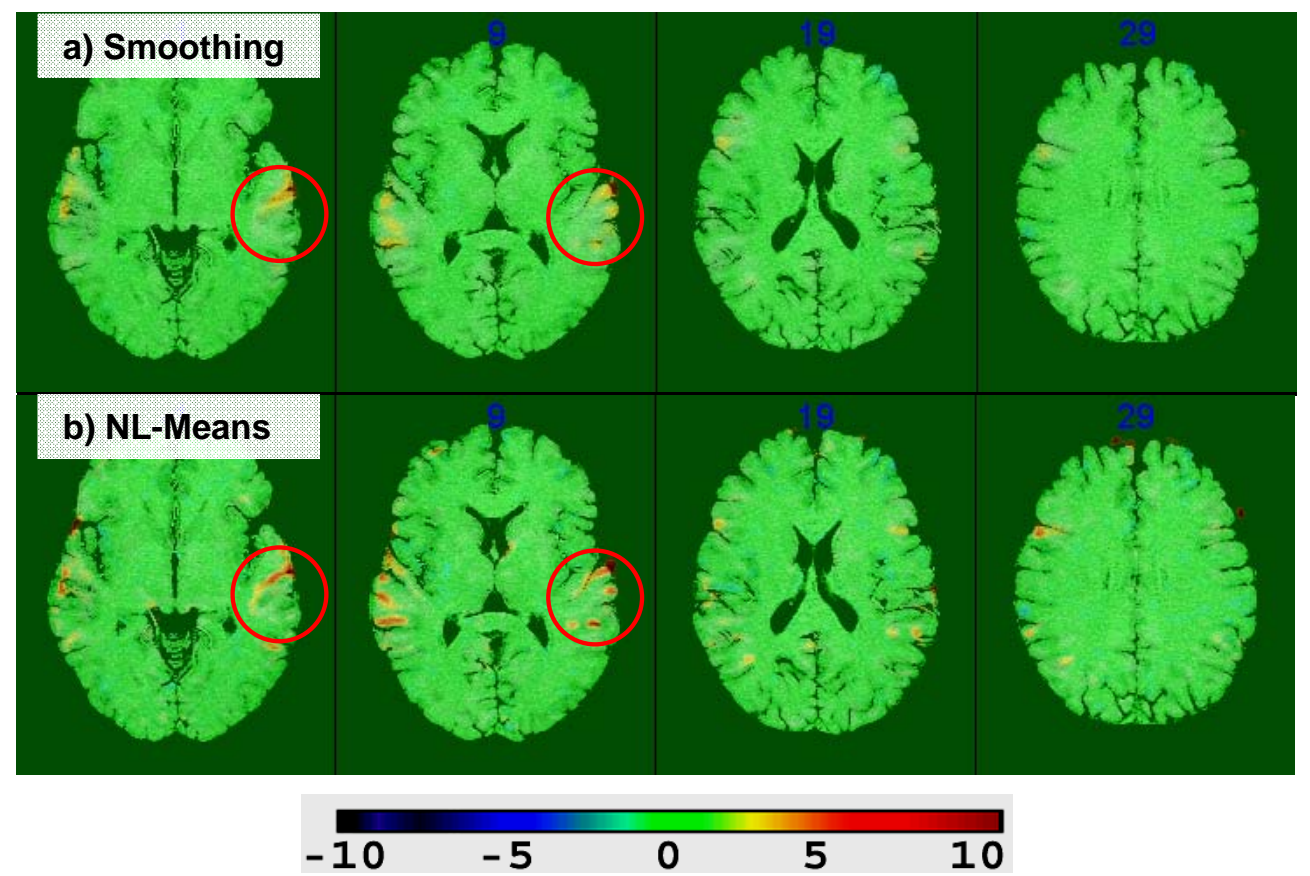

Figure 22. Schizophrenic patient with auditory hallucinations. Activation areas estimation is shown when a) Spatial smoothing and b) NL-Means methods have been applied. Red circles show activation areas that, in the case of the NL-Means, follow gyral structures more accurately than Spatial smoothing.
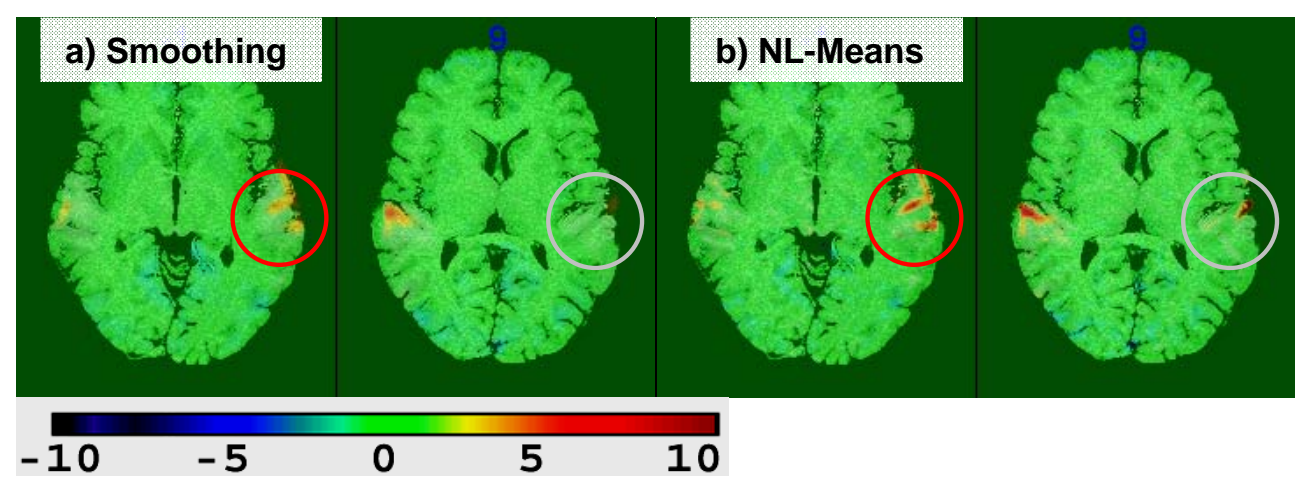

Figure 23. Healthy subject. Activation estimated areas overlaid on two axial slices. Red circles show the recovery of the activation areas with spatial smoothing and NL-Means. Gray circles highlight a difference in activation localization after spatial smoothing (outside the brain) and NL-Means (in the border of the brain). 
A cluster of activation is shown in Figure $\mathbf{2 4}$ in saggital, coronal and axial planes. Better localization and higher contrast estimation is obtained by means of the NL-Means method. However, localization enhancements pose new problems to inter-subject statistical analyses. Basically, two are the main difficulties that should be faced in order to perform group statistics over NL-Means filtered data:

- Inter-subject anatomical differences.

- Inter-subject functional localization differences.

Both problems are avoided when smoothing is applied by spatially blurring the data, at the expense of a loss in resolution. As an example, the Heschl's Gyrus $(H G)$ is a structure transverse to the Superior Temporal Lobe. Even though HG is described as the functional center of the Primary Auditory Cortex, its shape is highly variable between healthy subjects. Furthermore, the HG bifurcates into more than one gyrus in many healthy subjects (Devlin, Poldrack, 2007). Localization of the primary auditory function is also variable inside the gyrus between subjects, even though restricted to its posterior-medial part (Liegeois-Chauvel et al, 1991). Thus, the application of group analyses to NL-Means filtered fMRI data should be carefully studied and methods for these analyses should be proposed, that are the future research guidelines presented in the following chapter. 


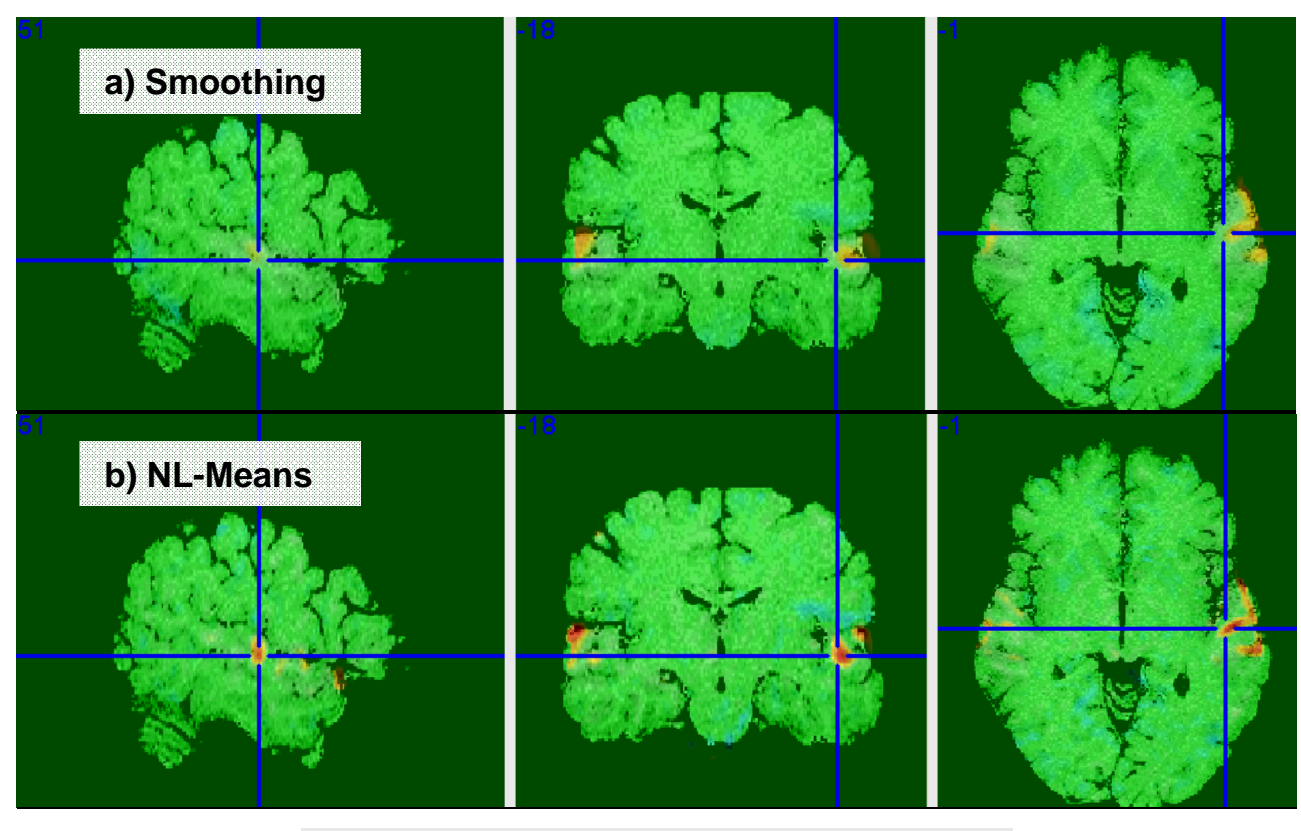

$$
\begin{array}{lllll}
-10 & -5 & 0 & 5 & 10
\end{array}
$$

Figure 24. Schizophrenic subject, saggital, coronal and axial views, respectively. Estimated activation areas overlaid on the subject's anatomical MR images, after a) Spatial smoothing and b) NL-Means. Blue crosshairs show the localization of one single voxel. NL-Means detected the narrow (see axial slice) activation while Spatial smoothing would mask the activation in the location of the voxel. 


\section{Concluding remarks and future}

research direction

"A man paints with his brains and not with his hands" - Michelangelo Buonarroti (1475 - 1564)

\section{7.a. - Compilation of results}

Chapter 3 to Chapter 6 show results from the PhD thesis separately. This section presents a compendium of the results obtained in the thesis.

1. Data analysis was applied to the fMRI data obtained with the emotional / nonemotional semantic auditory paradigm, in ten healthy control subjects.

1.1. Clear differentiation was observed in the group activation maps depending on the type of stimulus applied (emotional or non-emotional) in the intensity of activation (right and left temporal cortex), with higher intensity for the activation due to emotional content (see Figure 25).

1.2. In the same sample of subjects, the activation of specific areas (right precentral and supramarginal gyri; see Figure 25) was only present with the emotional stimuli, when the emotional semantic auditory paradigm was applied, but it was not present when the applied stimuli were nonemotional.

2. The same emotional / non-emotional semantic auditory paradigm was applied to evaluate cerebral activation by means of $\mathrm{fMRI}$ in 11 patients with schizophrenia with persistent hallucinations and 10 healthy subjects. 
2.1. Non-emotional-words-against-rest-baseline patients' group map did not show any activation with the common threshold applied (corrected $\mathrm{P}<0.05)$ when non-emotional words were presented. After application of a less stringent $P$ value $(P<0.001)$ in order to see activation trends, main areas involved were left middle and left superior temporal gyrus, middle cingulate gyrus and left inferior frontal gyrus at the orbital part (see Figure 26).

2.2. When patients were presented emotional content stimuli, activation survived the $\mathrm{P}<0.05$ corrected threshold and was greater both in extent and in estimated intensity. Middle left temporal gyrus was the main involved area. Other areas included right superior temporal gyrus, left and right insula, right median cingulate, right and left posterior cingulate, right amygdala, right and left middle frontal cortex at the orbital part, right inferior frontal cortex at the orbital area, and superior medial cortex.

2.3. Differences in activation in control subjects (see 1 ) were less marked than those in schizophrenic patients (2.1 and 2.2).

3. In a larger sample of 21 auditory hallucinating schizophrenic patients and 10 healthy paired subjects, differences in activation between healthy subjects and schizophrenic patients, and between non-emotional and emotional content, were estimated and encoded in a parametric map (fMRI map). Gray matter density decrement was also assessed comparing gray matter density between healthy and schizophrenic subjects (VBM map).

3.1. The fMRI map included right temporal middle, left temporal middle, right superior temporal and Heschl, right superomedial frontal, right angular, right posterior cingulum, left middle cingulum, and right thalamus.

3.2. The VBM map included left insula, right lingual, left postcentral, right precuneus, right insula, right superomedial frontal, left lingual and left middle temporal. 
3.3. All areas that appeared in the fMRI map and in the VBM map were reported. Large coinciding brain clusters in the fMRI map and in the VBM gray matter decreases maps $(P<0.005)$ were found in the left and right middle temporal and superior temporal gyri. Smaller coinciding clusters were found in the left posterior and right anterior cingular gyri, left inferior frontal gyrus, and middle occipital gyrus (see Figure 27).

4. NL-Means method, which was evaluated in synthetic and clinical data, enhanced the fMRI signal, by decreasing the noise level, without introducing noticeable blurring effects, as demonstrated by its better specificity and sensitivity in detecting activated voxels, especially of brain activation in areas of bordering sharp transitions, compared to the Gaussian Filter and the Adaptive Bilateral Filter.

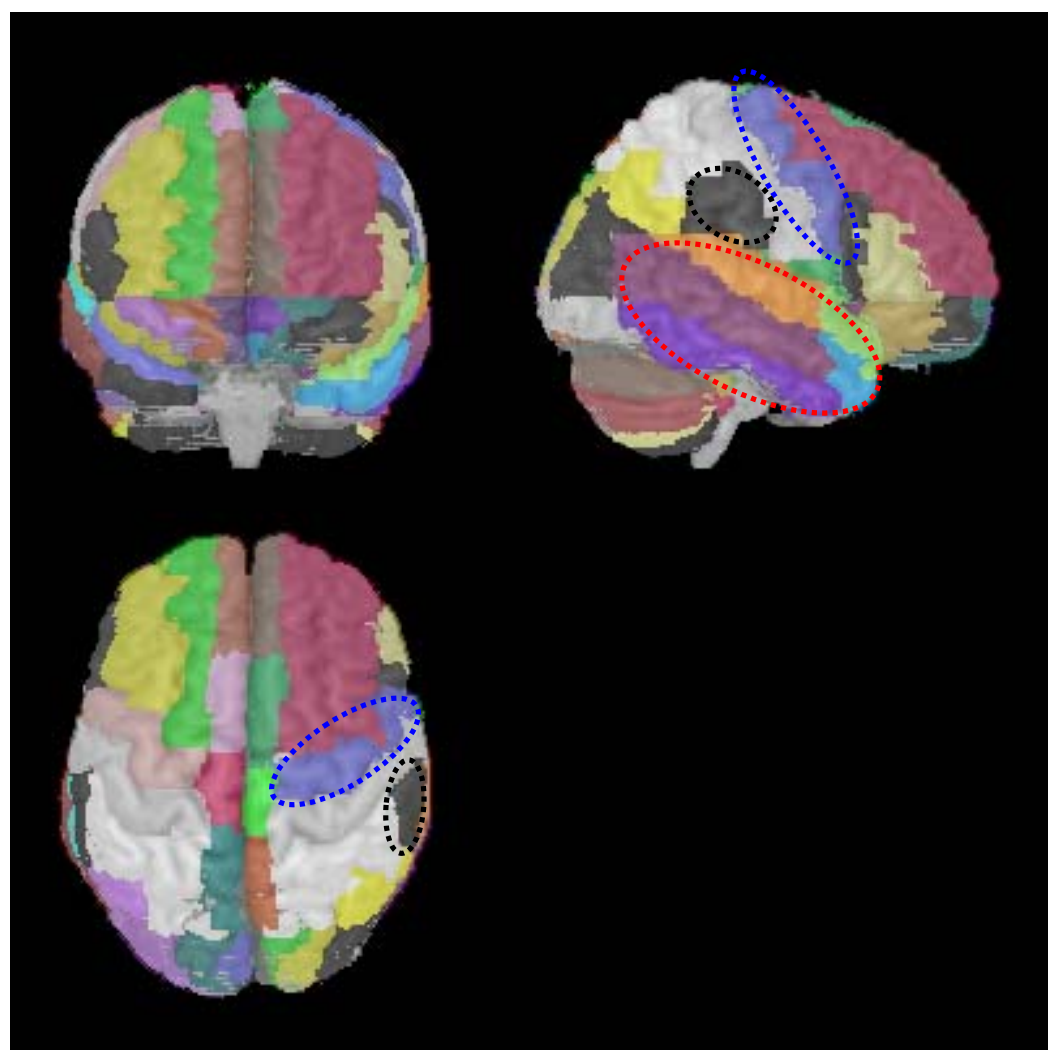

Figure 25. Brain rendering with functional brain areas delineated (coronal, axial and sagittal planes). Areas in 7.a.1 appear marked with dotted circles. Temporal cortex, red circle (Superior: orange; Middle: dark purple; Inferior: bright purple). 
Precentral gyrus, blue circle (blue colour; marked in axial and sagittal views). Supramarginal gyrus, black circle (black color; marked in axial and sagittal views).

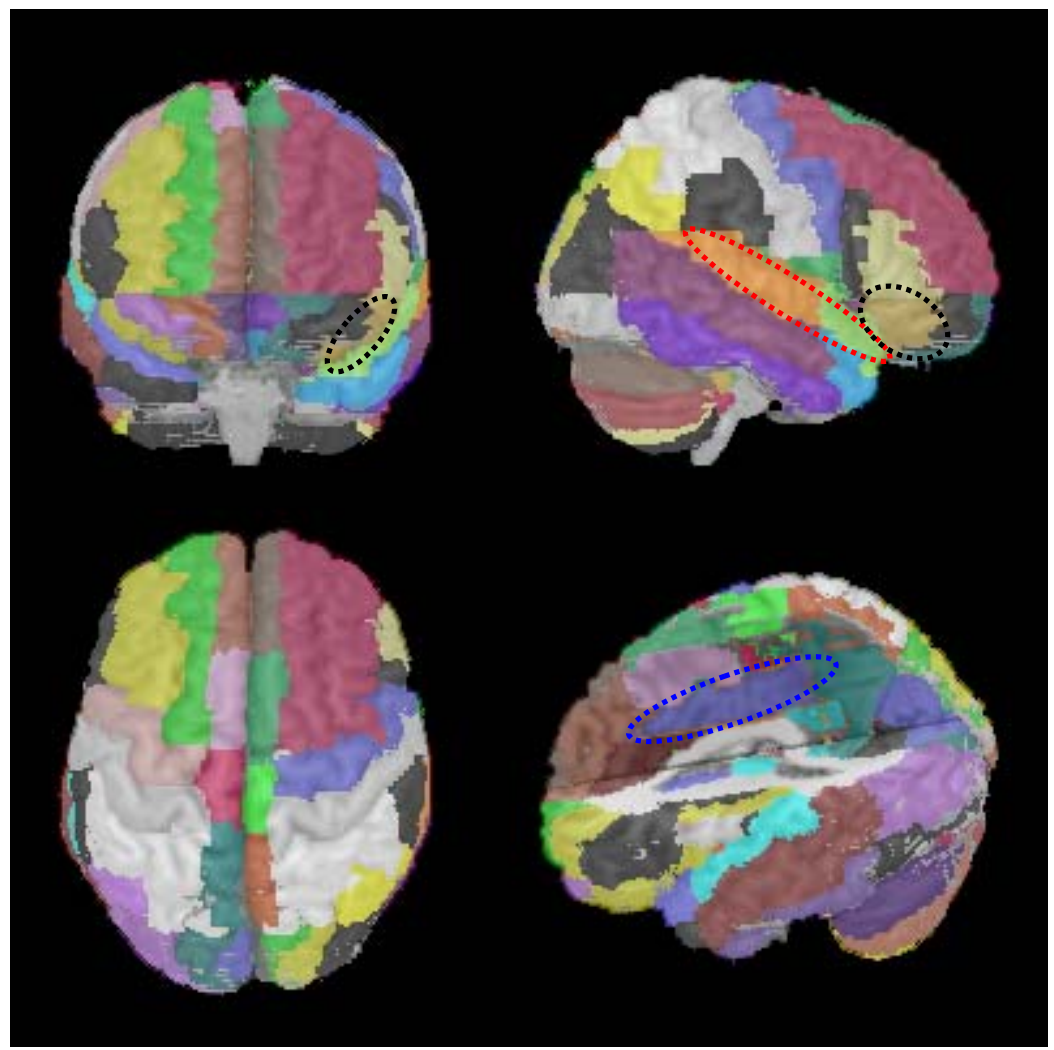

Figure 26. Brain rendering with functional brain areas delineated (coronal, axial, sagittal and sagittal-with-cutout planes). Areas in 7.a.2 section appear marked with dotted circles. Superior temporal gyrus, red circle (area with orange colour). Middle cingulate gyrus, blue circle (navy blue colour area). Inferior frontal orbital gyrus, black circle (golden colour area). 


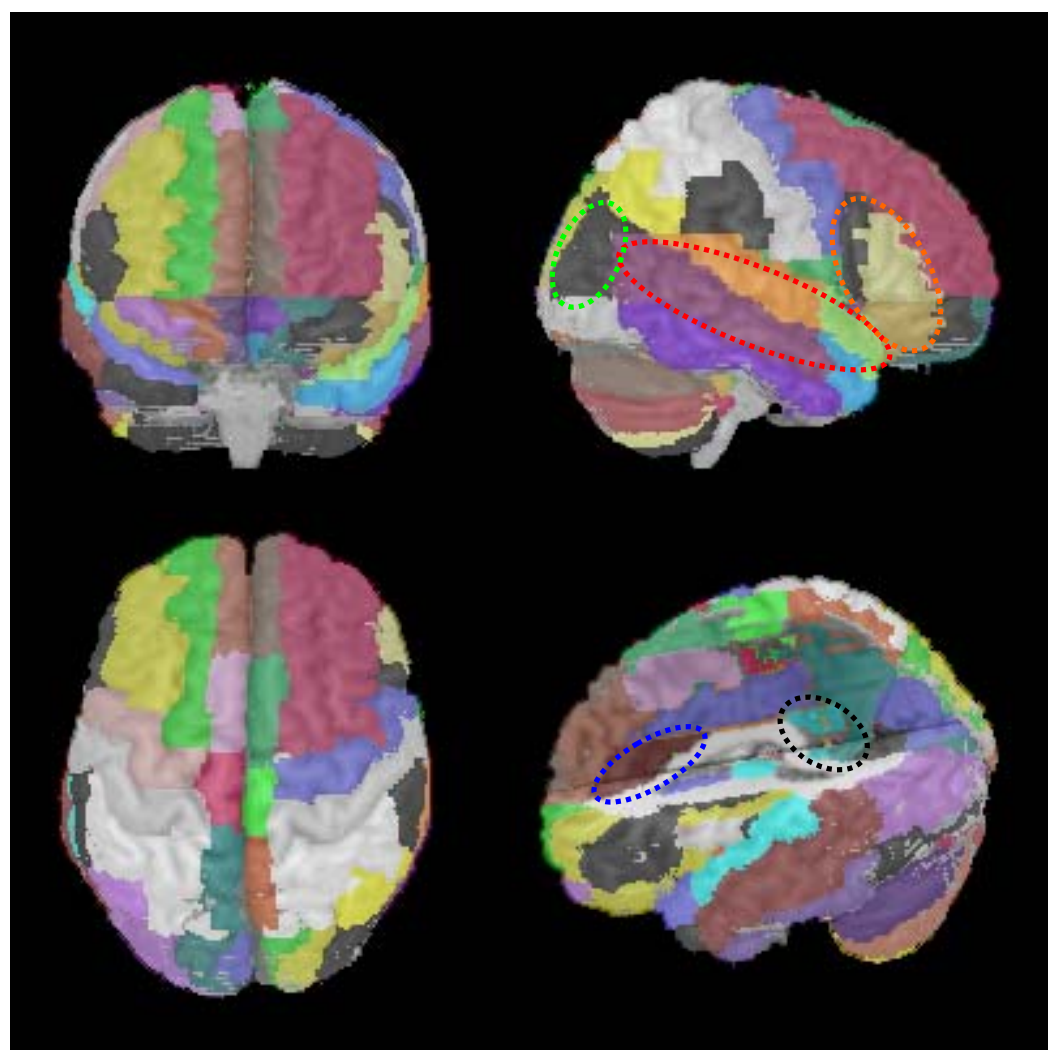

Figure 27. Brain rendering with functional brain areas delineated (coronal, axial, sagittal and sagittal-with-cutout planes). Areas in 7.a.3.3 section appear marked with dotted circles. Superior and middle temporal gyrus, red circle (areas with orange and dark purple colour, respectively). Inferior frontal gyrus, orange circle (opercular, triangular and orbital correspond to black, light yellow and golden colours, respectively). Middle occipital gyrus, green dotted circle (black colour area). Cingulate areas are shown marked with black (posterior) and blue (anterior) dotted circles. 


\section{7.b. - Conclusion}

The main outcomes from this thesis let us conclude:

Length and temporal distribution of the stimuli in the fMRI paradigm have been optimized to observe auditory semantic emotional processing in the schizophrenia research project.

The fMRI paradigm was successfully applied to a) healthy subjects, b) schizophrenic patients with auditory hallucinations and c) schizophrenic patients without auditory hallucinations. The processing in each subject's fMRI data, including fMRI data quality assurance, pre-processing and individual statistical analysis, let us perform group statistical tests that led us to find differences between groups of subjects a), b) and c).

Areas of activation for auditory semantic emotional processing were detected for each one of the three groups in a Random Effects fashion. Thus, the results are extensible to the populations respective of each group of subjects that have been analyzed.

Coincidence maps showing gray matter density decreases and activation differences (emotional compared to non-emotional) in auditory hallucinating schizophrenic patients and in healthy subjects were obtained. Areas with both a decrease in gray matter density in schizophrenic patients and functional differences were obtained. The detected areas show differences between healthy subjects and schizophrenic patients. Eight areas were detected to be different both by means of VBM and fMRI. Those areas may be of relevant interest in the study of schizophrenia. 
A new filtering method has been proposed for $\mathrm{fMRI}$ data filtering. Its main advantage is that it is respectful with the noise distribution present in the data and it obtains better signal recovery properties than nowadays commonly used filtering methods. The NL-Means filter has proved to decrease the noise level without suffering noticeable blurring effects, as demonstrated by its high specificity and sensitivity in detecting activated voxels, especially in bordering sharp transitions of brain activation areas. 


\section{7.b. - Future research direction}

The future work will be centred on the following topics:

Genetic and fMRI data crossing for the schizophrenia research project. Genetic characterization in a subject is known to mark his predisposition to schizophrenia illness. Studies about interaction of schizophrenia symptoms and genetic characteristics are a future research direction that has been already started (see Publications section). The union between genomics and biomedical imaging has been called imaging genomics by Hariri and Weinberger, and it is a very promising research field.

Further analyses with schizophrenia fMRI data. The three groups, a) healthy subjects, b) schizophrenic patients with auditory hallucinations and c) schizophrenic patients without auditory hallucinations, will be submitted to analyses of differences of activation between them that could explain the relation of inclination to auditory hallucinations and differential auditory semantic emotion processing.

The NL-Means filtering method will be further developed by:

o Increasing its speed by means of parallel GPU programming

o Studying the advantages of the NL-Means method in multisubject fMRI analyses. Studying other kinds of spatial normalization (e.g. surface-based models) that could preserve the resolution in study groups, such as Freesurfer, ANIMAL, CARET, etc.

Exploration of multitechnique data, acquired in the schizophrenia research project. Diffusion, fMRI, Magnetic Transfer, structural 
images and MR spectroscopy from the same subjects are currently present and pose a challenge for conjoint studies. 


\section{Annex I. Abbreviations}

\section{Abbreviations}

3D

$\mathrm{AH}$

BOLD

BPRS

DSM-IV

EEG

EM

EPI

FDR

FLD

fMRI

fSNR

GAS

GPU

hrf

HG

$M L$

$\mathrm{MNI}$

MNI152

MPFC

MRI

NL-Means

PANSS

PC

PCA

PET

PSYRATS

ReML

RFT

RMSE

ROI

SD

SDD

SNR

SPECT

SPM

VBM
Three-dimensional

Auditory Hallucinations

Blood Oxygen Level Dependent

Brief Psychiatric Rating Scale

Diagnostic and Statistical Manual of Mental Disorders - Volume IV

Electroencelography

Expectation Maximization

Echo Planar Imaging

False Discovery Rate

Fisher's Linear Discriminant

functional Magnetic Resonance Imaging

functional SNR

Global Assessment Scale

Graphical Processing Unit

hemodynamic response function

Heschl's Gyrus

Maximum Likelihood estimation

Montreal Neurological Institute

Montreal Neurological Institute brain template

Medial Prefrontal Cortex

Magnetic Resonance Imaging

Non-Local Means

Positive and Negative Syndrome Scale

Principal Component

Principal Component Analysis

Positron Emission Tomography

Psychotic Symptom Rating Scale

Restricted Maximum Likelihood estimation

Random Field Theory

Root Mean Square Error

Region of Interest

Standard Deviation

Standard Deviation of the Difference

Signal-to-Noise Ratio

Single Photon Emission Computed Tomography

Statistical Parametric Map

Voxel-based morphometry 


\section{Annex 11. Publications}

\section{Peer reviewed journal publications}

García-Martí, G, Aguilar, EJ, Lull, JJ, Martí-Bonmatí, L, Escartí, María J, Manjón, JV, Moratal, D, Robles, M, Sanjuán, J. "Schizophrenia with auditory hallucinations: A voxel-based morphometry study." Progress in NeuroPsychopharmacology and Biological Psychiatry 32 (2008): 72-80.

Manjón, JV, Lull, JJ, Carbonell-Caballero, J, García-Martí, G, MartíBonmatí, L, Robles, M. "A nonparametric MRI inhomogeneity correction method." Medical Image Analysis 11 (2007): 336-45.

Manjón, JV, Carbonell-Caballero, J, Lull, JJ, García-Martí, G, MartíBonmatí, L, Robles, M. "MRI denoising using Non Local Means." Medical Image Analysis: In press.

Martí-Bonmatí, L, Lull, JJ, García-Martí, G, Aguilar, EJ, Moratal-Pérez, D, Poyatos, C, Robles, M, Sanjuán, J. "Chronic auditory hallucinations in schizophrenic patients: MR analysis of the coincidence between functional and morphologic abnormalities." Radiology 244 (2007): 549-56.

Sanjuán, J, Lull, JJ, Martí-Bonmatí, L, Aguilar, EJ, Gadea, M, Moratal, D, González, JC, Robles, M. "Emotional auditory paradigm in neuroimaging: a base for the study of psychosis." Actas españolas de psiquiatría 33 (2005): 383-9.

Sanjuan, J, Lull, JJ, Aguilar, EJ, Martí-Bonmatí, L, Moratal, D, González, JC, Robles, M, Keshavan, MS. "Emotional words induce enhanced brain activity in schizophrenic patients with auditory hallucinations." Psychiatry Research in Neuroimaging 154, no. 1 (2007): 21-9.

\section{International congress contributions}

Lull JJ, Manjon JV, Garcia-Marti G, Marti-Bonmati L, Robles M. Non local means filter enhances fMRI signal detection (POSTER)

CONFERENCE: European Congress of Radiology

PUBLICATION: Book of abstracts: 430.

2008

Lull, JJ, García-Martí, G, Robles, M, Sanjuán, J, Keshavan, MS, Diwadkar, V.

Hyper-activation of the middle temporal lobe in hallucinating schizophrenia patients is associated with reduced structural integrity: Preliminary convergent analysis of fMRI and MRI data. (POSTER) 
CONFERENCE: 12th Meeting of the Human Brain Mapping

PUBLICATION: Neurolmage 31/S1 (2006) S1-S242

2006

García-Martí, G, Aguilar, EJ, Lull, JJ, Martí-Bonmatí, L, Moratal, D, Manjón, JV, Robles, M, Sanjuán, J.

Auditory Hallucinations in Schizophrenia (POSTER).

CONFERENCE: XIIIth Biennial Winter Workshop on Schizophrenia Research

PUBLICATION: Schizophrenia Research. An International Multidisciplinary Journal 2006

L, Robles, M.

Manjón-Herrera, JV, Carbonell, J, Lull, JJ, Garcia-Martí, G, Martí-Bonmatí, A Generic Coarse to Fine MRI Inhomogeneity Correction Method (POSTER) CONFERENCE: 12th Meeting. Human Brain Mapping PUBLICATION: Neurolmage 31/S1 (2006) S1-S242 2006

Lull, N, García-Martí, G, Noé, E, de La Cueva, E, Chirivella, J, Ferri, J, Lull, JJ, Sopena, R, Robles, M.

Association between neurological outcome and thalamic metabolism in patients with traumatic brain injury: an FDG-PET study.

CONFERENCE: INS/SVNP/GNP Meeting Zurich.

PUBLICATION: JINS 2006(12): Supplement 2:pp69

2006

Navarro, A, Lull, JJ, Martí-Bonmatí, L, Garcia-Marti, G, Sanjuán, J, Manjón, JV, Ford, J, Aguilar, EJ Robles, M.

Discrimination of schizophrenic patients from controls by means of FLD applied to fMRI

CONFERENCE: European Society for Magnetic Resonance in Medicine and Biology 23rd Annual Scientific Meeting (ESMRMB).

PUBLICATION: MAGMA 19,Sup.1, 2006: pp317-318. ISSN: 1352-8661 2006

Garcia-Marti, G, Carbonell-Caballero, J, Marti-Bonmati, L, Manjon, JV, Lull, JJ, de la Iglesia, M, Moratal, D, Robles, M, Sanjuan, J.

Voxel-based diffusion: a temporal lobe analysis in schizophrenia CONFERENCE: ESMRMB 23rd Annual Scientific Meeting

PUBLICATION: MAGMA Vol 19,Sup.1, pp291. 2006. ISSN: 1352-8661 2006

Lull, JJ, Sanjuán, J, García-Martí, G, Martí-Bonmatí, L, Manjón, JV, Carbonell, J, Robles, M.

fMR amygdala findings in chronic hallucinatory schizophrenic patients.

CONFERENCE: ESMRMB 22nd Annual Scientific Meeting. Basle/CH PUBLICATION: book of abstracts 2005

Garcia-Marti, G, Lull, JJ, Marti-Bonmati, L, Sanjuán, J, Poyatos, C, Carbonell Carballero, J, Manjón, JV, Robles, M. 
Morphometry in schizophrenic patients with chronic auditory hallucinations CONFERENCE: ESMRMB 2005. 22nd Annual Scientific Meeting. Basle/CH PUBLICATION: book of abstracts 2005 González, JC.

Aguilar, EJ, Lull, JJ, Martí-Bonmatí, L, Sanjuán, J, Moratal, D, Robles, M, AUDITORY fMRI DIFFERENCES BETWEEN SCHIZOPHRENIC PATIENTS WITH AUDITORY HALLUCINATIONS AND CONTROL SUBJECTS ARE MAINLY RELATED TO THE EMOTIONAL CONTENT (POSTER) CONFERENCE: Congress on Schizophrenia Research. Savannah, Georgia, USA PUBLICATION: book of abstracts 2005

Manjón, JV, Lull, JJ, Carbonell, J, García, G, Martí-Bonmatí, L, Robles, M. Reducing Partial Volume Artifacts In MRI Segmentation (Manuscript) CONFERENCE: 3rd European Medical and Biological Engineering Conference (EMBEC) PUBLICATION: IFMBE Proceedings 11, 2005 2005 Robles, M.

Lull, JJ, Martí-Bonmatí, L., Moratal-Pérez, D, Vallés-Lluch, A, Manjón, JV, Optimal flip-angle choice in GRE-EPI fMRI (POSTER).

CONFERENCE: ESMRMB. 21st Annual Scientific Meeting PUBLICATION: MAGMA 2004; 17(S1); S249.

2004

Manjón, JV, Lull JJ, Navarro, A, Carbonell, J, Martí-Bonmatí, L, Robles, M. Fast MRI Bias Correction (POSTER) CONFERENCE: ESMRMB. 21st Annual Scientific Meeting PUBLICATION: MAGMA 2004; 17(S1); S249. 2004

Carbonell, J, Martí-Bonmatí, L, de La Iglesia, M, Lull, JJ, Manjón, JV, Robles, M, Moratal-Pérez, D, Casanova, B, Coret, F. Computerized medulla area calculation: methodology and application to Multiple Sclerosis (POSTER)

CONFERENCE: ESMRMB. 21st Annual Scientific Meeting PUBLICATION: MAGMA 2004; 17(S1); S247.

2004

Coello, SC, Moratal-Pérez, D, Castells, F, Lull, JJ, Martí-Bonmatí, L, Sanjuán, J, Millet-Roig, J.

fMRI data analysis on schizophrenic patients under auditory stimuli. A comparison between the General Linear Model (GLM) and Independent Component Analysis (ICA) (POSTER)

CONFERENCE: ESMRMB. 21st Annual Scientific Meeting.

PUBLICATION: MAGMA 2004; 17(S1); S186.

2004 


\section{Annex 11. References}

Aleman, A, Kahn, RS. 2005. Strange feelings: do amygdala abnormalities dysregulate the emotional brain in schizophrenia? Progress in neurobiology 77(5):283-98.

Algarabel S. 1996. Indices de interes psicolinguistico de 1.917 palabras castellanas Psycholinguistic indexes of 1,917 Spanish words. Cognitiva 8:43-88(46).

Aguirre, GK, Zarahn, E, D'Esposito, M. 1997. Empirical Analyses of BOLD fMRI Statistics. Neurolmage 5(3):199-212.

Allen P, Laroi F, McGuire PK, Aleman A. 2008. The hallucinating brain: A review of structural and functional neuroimaging studies of hallucinations. Neuroscience \& Biobehavioral Reviews 32:175-191.

Andersson JLR, Hutton C, Ashburner J, Turner R, Friston K. 2001. Modeling Geometric Deformations in EPI Time Series. Neurolmage 13:903-919.

Andreasen, NC. 1982. Negative symptoms in schizophrenia. Definition and reliability. Archives of general psychiatry 39(7):784-8.

Ardekani, B, Guckemus, S, Bachman, A, Hoptman, M, Wojtaszek, M, Nierenberg, J. 2005. Quantitative comparison of algorithms for inter-subject registration of 3D volumetric brain MRI scans. Journal of Neuroscience Methods 142(1):67-76.

Ashburner J, Friston KJ. 2001. Why Voxel-Based Morphometry Should Be Used. Neurolmage 14:1238-1243.

Ashburner, J, Friston, K. 1997. Multimodal image coregistration and partitioning--a unified framework. Neuroimage 6(3):209-17.

Ashburner J, Friston KJ. 1999. Nonlinear spatial normalization using basis functions. Human Brain Mapping 7:254-26.

Bandettini, P. 2007. Functional MRI today. International journal of psychophysiology : official journal of the International Organization of Psychophysiology 63(2):138-45.

Benjamini Y, Hochberg Y. 1995. Controlling the False Discovery Rate: A Practical and Powerful Approach to Multiple Testing. Journal of the Royal Statistical Society 57:289-300 
Binder, JR, Frost, JA, Hammeke, TA, Bellgowan, PSF, Springer, JA, Kaufman, JN, Possing, ET. 2000. Human Temporal Lobe Activation by Speech and Nonspeech Sounds. Cerebral Cortex 10(5): 512-528.

Birn, RM, Saad, ZS, Bandettini. PA. 2001. Spatial heterogeneity of the nonlinear dynamics in the FMRI BOLD response. Neurolmage 14(4):817-26.

Bookstein FL (2001) "Voxel-Based Morphometry" Should Not Be Used with Imperfectly Registered Images. Neurolmage 14:1454-1462

Box G, Jenkins GM, Reinsel G. 1994. Time Series Analysis: Forecasting \& Control 3rd ed. Prentice Hall.

Boynton, GM, Engel, SA, Glover, GH, Heeger, DJ. 1996. Linear systems analysis of functional magnetic resonance imaging in human V1. The Journal of neuroscience : the official journal of the Society for Neuroscience 16(13):4207-21.

Buades, A, Coll, B, Morel, JM. 2005. A Review of Image Denoising Algorithms, with a New One. Multiscale Modeling \& Simulation 4(2):490-530.

Buxton RB, Wong EC, Frank LR. 1998. Dynamics of blood flow and oxygenation changes during brain activation: the balloon model. Magnetic Resonance in Medicine 39:855-64.

Casanova R, Srikanth R, Baer A, Laurienti PJ, Burdette JH, Hayasaka S, Flowers L, Wood F, Maldjian JA. 2007. Biological parametric mapping: A statistical toolbox for multimodality brain image analysis. Neurolmage 34:137-143

Coello S, Moratal D, Castells F, Lull JJ, Marti-Bonmati L, Sanjuan J, Millet-Roig J. 2004. fMRI data analysis on schizophrenic patients under auditory stimuli. A comparison between the General Linear Model (GLM) and Independent Component Analysis (ICA). Magma 17:S186.

Dale AM, Fischl B, Sereno MI. 1999. Cortical Surface-Based Analysis: I. Segmentation and Surface Reconstruction. Neurolmage 9:179-194

Desmond, JE, Atlas, SW. 2000. Task-correlated Head Movement in fMR Imaging: False Activations Can Contaminate Results Despite Motion Correction. AJNR American Journal of Neuroradiology 21(8):1370-1371.

Devlin JT, Poldrack RA. 2007. In praise of tedious anatomy. Neurolmage 37:10331041.

Essen DC Van, Drury HA, Dickson J, Harwell J, Hanlon D, Anderson CH. 2001. An Integrated Software Suite for Surface-based Analyses of Cerebral Cortex. J Am Med Inform Assoc. 8:443-459

Fakra E, Salgado-Pineda P, Delaveau P, Hariri AR, Blin O. 2008. Neural bases of different cognitive strategies for facial affect processing in schizophrenia. Schizophr Res 100:191-205. 
Fan Y, Rao H, Hurt H, Giannetta J, Korczykowski M, Shera D, Avants BB, Gee JC, Wang J, Shen D. 2007. Multivariate examination of brain abnormality using both structural and functional MRI. Neurolmage 36:1189-1199

Ford J, Farid H, Makedon F, Flashman L, McAllister T, Megalooikonomou V, Saykin A. 2003. Patient Classification of fMRI Activation Maps. Presented at the 6th Annual International Conference on Medical Image Computing and Computer Assisted Intervention (MICCAI'O3).

Frackowiak, RS, Ashburner, JT, Penny, WD, Zeki, S. 2003. Human Brain Function, Second Edition. Ed. Academic Press.

Freeman, D, Garety PA. 2003. Connecting neurosis and psychosis: the direct influence of emotion on delusions and hallucinations. Behaviour research and therapy 41(8):923-47.

Friman, O, Carlsson J, Lundberg P, Borga M, Knutsson H. 2001. Detection of neural activity in functional MRI using canonical correlation analysis. Magnetic Resonance in Medicine 45(2):323-330.

Friston, KJ, Ashburner, J, Frith, CD, Poline, J, Heather, JD, Frackowiak, RSJ. 1995. Spatial registration and normalization of images. Human Brain Mapping 3(3):165-189.

Friston KJ, Penny W, Phillips C, Kiebel S, Hinton G, Ashburner J. 2002. Classical and Bayesian inference in neuroimaging: theory. Neuroimage 16:465-83.

Friston KJ, Zarahn E, Josephs O, Henson RNA, Dale AM. 1999. Stochastic Designs in Event-Related fMRI. Neurolmage 10:607-619.

Garcia-Marti G, Aguilar EJ, Lull JJ, Marti-Bonmati L, Escarti MJ, Manjon JV, Moratal D, Robles M, Sanjuan J. 2008. Schizophrenia with auditory hallucinations: A voxel-based morphometry study. Progress in NeuroPsychopharmacology and Biological Psychiatry 32:72-80.

Genovese, CR, Lazar, NA, Nichols, T. 2002. Thresholding of statistical maps in functional neuroimaging using the false discovery rate. Neuroimage 15(4):870-8.

Geschwind, N. 1979. Specializations of the Human Brain. Scientific American 241:180-99.

Harrison PJ. 2005. Neuropathology of schizophrenia. Psychiatry 4:18-21.

Kapur S. 2003. Psychosis as a State of Aberrant Salience: A Framework Linking Biology, Phenomenology, and Pharmacology in Schizophrenia. Am J Psychiatry 160:13-23. 
Laviolette, SR. 2007. Dopamine modulation of emotional processing in cortical and subcortical neural circuits: evidence for a final common pathway in schizophrenia?. Schizophrenia bulletin 33(4):971-81.

Lee K, Brown WH, Egleston PN, Green RDJ, Farrow TFD, Hunter MD, Parks RW, Wilkinson ID, Spence SA, Woodruff PWR. 2006. A functional magnetic resonance imaging study of social cognition in schizophrenia during an acute episode and after recovery. Am J Psychiatry 163:1926-33.

Liegois-Chauvel C, Musolino A, Chauvel P. 1991. Localization of the Primary Auditory Area in Man. Brain 114A:139-153

Locascio JJ, Jennings PJ, Moore Cl, \& Corkin S. 1997. Time series analysis in the time domain and resampling methods for studies of functional magnetic resonance brain imaging. Human Brain Mapping 5:168-193.

Logothetis, NK, Pauls, J, Augath, M, Trinath, T, Oeltermann, A. 2001. Neurophysiological investigation of the basis of the fMRI signal. Nature 412(6843):150-7.

Lull JJ, Garcia-Marti G, Robles M, Sanjuan J, Keshavan MS, Diwadkar V. 2006. Hyper-activation of the middle temporal lobe in hallucinating schizophrenia patients is associated with reduced structural integrity: Preliminary convergent analysis of fMRI and MRI data. Presented at the 12th Meeting of the Human Brain Mapping. Neurolmage 31/S1 (2006) S1-S242.

Lull, JJ, Martí-Bonmatí, L., Moratal-Pérez, D, Vallés-Lluch, A, Manjón, JV, Robles, M. 2004. Optimal flip-angle choice in GRE-EPI fMRI. MAGMA 17(S1): S249.

Lütkenhöner B, Krumbholz K, Lammertmann C, Seither-Preisler A, Steinsträter O, Patterson RD. 2003. Localization of Primary Auditory Cortex in Humans by Magnetoencephalography. Neurolmage 18:58-66

Lynam, DR, Widiger, TA. 2007. Using a general model of personality to identify the basic elements of psychopathy. Journal of personality disorders 21(2):16078.

Manjón, JV, Carbonell-Caballero, J, Lull, JJ, García-Martí, G, Martí-Bonmatí, L, Robles, M. 2008. MRI denoising using Non Local Means. Medical Image Analysis: In press.

Mansfield, P. 1977. Multi-planar image formation using NMR spin echoes. Journal of Physics C: Solid State Physics 10(3):L55-L58.

Menon RR, Barta PE, Aylward EH, Richards SS, Vaughn DD, Tien AY, Harris GJ, Pearlson GD. 1995. Posterior superior temporal gyrus in schizophrenia: grey matter changes and clinical correlates. Schizophrenia Research 16:127-135. 
Mckeown, MJ, Makeig, S, Brown, G, Jung, T, Kindermann, S, Bell, A, Sejnowski, TJ. 1998. Analysis of $\mathrm{fMRI}$ data by blind separation into independent spatial components. Human Brain Mapping 6(3):160-188.

Mendrek A, Mancini-Marië A, Fahim C, Stip E. 2007. Sex differences in the cerebral function associated with processing of aversive stimuli by schizophrenia patients. Aust N Z J Psychiatry 41:136-41.

Menon, RS, Goodyear, BG. 1999. Submillimeter functional localization in human striate cortex using BOLD contrast at 4 Tesla: implications for the vascular point-spread function. Magnetic resonance in medicine 41(2):230-5.

Morosan P, Rademacher J, Schleicher A, Amunts K, Schormann T, Zilles K. 2001. Human Primary Auditory Cortex: Cytoarchitectonic Subdivisions and Mapping into a Spatial Reference System. Neurolmage 13:684-701

Murphy, FC, Nimmo-Smith, I, Lawrence, AD. 2003. Functional neuroanatomy of emotions: a meta-analysis. Cognitive, affective \& behavioral neuroscience 3(3):207-33.

Navarro A, Lull JJ, Marti-Bonmati L, Garcia-Marti G, Sanjuan J, Ford J, Aguilar EJ, \& Robles M (2006) Discrimination of schizophrenic patients from controls by means of FLD applied to fMRI. Magma (New York, N.Y.) 16, S1:317318.

Nichols, T, Hayasaka, S. 2003. Controlling the familywise error rate in functional neuroimaging: a comparative review. Statistical Methods in Medical Research 12(5):419-46

Niznikiewicz MA, Kubicki M, Shenton ME. 2003. Recent structural and functional imaging findings in schizophrenia. Current Opinion in Psychiatry 16:123.

Oakes, TR, Johnstone T, Ores Walsh, KS, Greischar, LL, Alexander, AL, Fox, AS, Davidson, RJ. 2003. Comparison of fMRI motion correction software tools. Neurolmage 28(3):529-43.

Ogawa, S Lee, T, Kay, A, Tank, D. 1990. Brain magnetic resonance imaging with contrast dependent on blood oxygenation. Proceedings of the National Academy of Sciences of the United States of America 87(24):9868-72.

Ogawa, S Menon, R, Tank, D, Kim, SG, Merkle, H, Ellermann, J, Ugurbil, K. 1993. Functional brain mapping by blood oxygenation level-dependent contrast magnetic resonance imaging. A comparison of signal characteristics with a biophysical model. Biophysical journal 64(3):803-12.

Perneger TV . 1998 What's wrong with Bonferroni adjustments. BMJ 316:12361238

Phillips, ML. 2003. Understanding the neurobiology of emotion perception: implications for psychiatry. British Journal of Psychiatry:190-2. 
Plaze M, Bartrés-Faz D, Martinot J, Januel D, Bellivier F, De Beaurepaire R, Chanraud S, Andoh J, Lefaucheur J, Artiges E, Pallier C, Paillère-Martinot M. 2006. Left superior temporal gyrus activation during sentence perception negatively correlates with auditory hallucination severity in schizophrenia patients. Schizophr Res 87:109-15.

Reimold, M, Slifstein, M, Heinz, A, Mueller-Schauenburg, W, Bares, R. 2006. Effect of spatial smoothing on t-maps: arguments for going back from t-maps to masked contrast images. Journal of cerebral blood flow and metabolism : official journal of the International Society of Cerebral Blood Flow and Metabolism 26(6):751-9.

Rorden C, Brett M. 2000. Stereotaxic display of brain lesions. Behav Neurol 12:191-200.

Roy, CS, Sherrington, CS. 1890. On the Regulation of the Blood-supply of the Brain. The Journal of physiology 11(1-2):85-158.17.

Sabbah P, Foehrenbach H, Dutertre G, Nioche C, DeDreuille O, Bellegou N, Mangin JF, Levöque C, Faillot T, Gaillard JF, Desgeorges M, Cordoliani YS. 2002. Multimodal anatomic, functional, and metabolic brain imaging for tumor resection. Clinical Imaging 26:6-12.

Sanjuan J, Lull JJ, Aguilar EJ, Martí-Bonmatí L, Moratal D, Gonzalez JC, Robles M, Keshavan MS. 2007. Emotional words induce enhanced brain activity in schizophrenic patients with auditory hallucinations. Psychiatry Res 154:219.

Shenton ME, Kikinis R, Jolesz FA, Pollak SD, LeMay M, Wible CG, Hokama H, Martin J, Metcalf D, Coleman M. 1992. Abnormalities of the left temporal lobe and thought disorder in schizophrenia. A quantitative magnetic resonance imaging study. $N$ Engl J Med 327:604-12.

Schultz W. 2007. Multiple dopamine functions at different time courses. Annu Rev Neurosci 30:259-88.

Sunaert, S. 2006. Presurgical planning for tumor resectioning. Journal of magnetic resonance imaging : Journal of Magnetic Resonance Imaging 23(6):887905.

Taylor JL, Blanton RE, Levitt JG, Caplan R, Nobel D, Toga AW. 2005. Superior temporal gyrus differences in childhood-onset schizophrenia. Schizophrenia Research 73:235-241.

Tervaniemi M. Hugdahl K. 2003. Lateralization of auditory-cortex functions. Brain Research Reviews 43:231-246

Tracy, DK, Shergill SS. 2006. Imaging auditory hallucinations in schizophrenia. Acta Neuropsychiatrica 18(2):71-78. 
Tzourio-Mazoyer, N, Landeau, B, Papathanassiou, D, Crivello, F, Etard, O, Delcroix, N, Mazoyer, B, Joliot, M. 2002. Automated anatomical labeling of activations in SPM using a macroscopic anatomical parcellation of the MNI MRI single-subject brain. Neuroimage 15(1):273-89.

Vazquez, AL, Noll, DC. 1998.Nonlinear aspects of the BOLD response in functional MRI. Neurolmage 7(2):108-18.

Williams LM, Das P, Liddell BJ, Olivieri G, Peduto AS, David AS, Gordon E, Harris AWF. 2007. Fronto-limbic and autonomic disjunctions to negative emotion distinguish schizophrenia subtypes. Psychiatry Res 155:29-44.

Wink, AM, Roerdink, JBT. 2004. Denoising functional MR images: a comparison of wavelet denoising and Gaussian smoothing. IEEE transactions on medical imaging 23(3):374-87.

Worsley KJ, Evans AC, Marrett S, Neelin P. 1992. A three-dimensional statistical analysis for CBF activation studies in human brain. J Cereb Blood Flow Metab 12:900-18.

Worsley, KJ, Marrett, S, Neelin, P, Vandal, AC, Friston, KJ, Evans, AC. 1996. A unified statistical approach for determining significant signals in images of cerebral activation. Human Brain Mapping 4(1):58-73.

Woods, RP, Grafton, ST, Watson, JD, Sicotte, NL, Mazziotta, JC. 1998. Automated image registration: II. Intersubject validation of linear and nonlinear models. J Comput Assist Tomogr 22:153-65

Zarahn, E, Aguirre, GK, D'Esposito, M. 1997. Empirical analyses of BOLD fMRI statistics. I. Spatially unsmoothed data collected under null-hypothesis conditions. Neurolmage 5(3):179-97. 